\title{
Equilibrium Configurations for Epitaxially Strained Films and Material Voids in Three-Dimensional Linear Elasticity
}

\author{
Vito CRismale(D) \& Manuel Friedrich
}

\author{
Communicated by I. FONSECA
}

\begin{abstract}
We extend the results about the existence of minimizers, relaxation, and approximation proven by Bonnetier AND Chambolle (SIAM J Appl Math 62:10931121, 2002), Chambolle ANd Solci (SIAM J Math Anal 39:77-102, 2007) for an energy related to epitaxially strained crystalline films, and by BRAIDES et al. (ESAIM Control Optim Calc Var 13:717-734, 2007) for a class of energies defined on pairs of function-set. We study these models in the framework of threedimensional linear elasticity, where a major obstacle to overcome is the lack of any a priori assumption on the integrability properties of displacements. As a key tool for the proofs, we introduce a new notion of convergence for $(d-1)$-rectifiable sets that are jumps of $G S B D^{p}$ functions, called $\sigma_{\mathrm{sym}}^{p}$-convergence.
\end{abstract}

\section{Introduction}

The last years years have witnessed a remarkable progress in the mathematical and physical literature towards the understanding of stress driven rearrangement instabilities (SDRI), that is, morphological instabilities of interfaces between elastic phases generated by the competition between elastic and surface energies of (isotropic or anisotropic) perimeter type. Such phenomena are for instance observed in the formation of material voids inside elastically stressed solids. Another example is hetero-epitaxial growth of elastic thin films, when thin layers of highly strained hetero-systems, such as InGaAs/GaAs or SiGe/Si, are deposited onto a substrate: in case of a mismatch between the lattice parameters of the two crystalline solids, the free surface of the film is flat until a critical value of the thickness is reached, after which the free surface becomes corrugated (see for example $[4,46,49,50,56,58]$ for some physical and numerical literature).

From a mathematical point of view, the common feature of functionals describing SDRI is the presence of both stored elastic bulk and surface energies. In 
the static setting, problems arise concerning existence, regularity, and stability of equilibrium configurations obtained by energy minimization. The analysis of these issues is by now mostly developed in dimension two only.

Starting with the seminal work by Bonnetier and CHambolle [9] who proved existence of equilibrium configurations, several results have been obtained in $[5,7,35,37,45,48]$ for hetero-epitaxially strained elastic thin films in $2 \mathrm{D}$. We also refer to $[29,30,53]$ for related energies and to [52] for a unified model for SDRI. In the three dimensional setting, results are limited to the geometrically nonlinear setting or to linear elasticity under an antiplane-shear assumption $[8,20]$. In a similar fashion, regarding the study of material voids in elastic solids, there are works about existence and regularity in dimension two [14,34] and a relaxation result in higher dimensions [11] for nonlinearly elastic energies or in linear elasticity under antiplane-shear assumption. Related to [11], we also mention a similar relaxation result in the presence of obstacles [33], and the study of homogenization in periodically perforated domains, $\mathrm{cf}$. for example $[13,32]$.

The goal of the present paper is to extend the results about relaxation, existence, and approximation obtained for energies related to material voids [11] and to epitaxial growth $[9,20]$, respectively, to the case of linear elasticity in arbitrary space dimensions. As already observed in [20], the main obstacle for deriving such generalizations lies in the fact that a deep understanding of the function space of generalized special functions of bounded deformation (GSBD) is necessary. Indeed, our strategy is based extensively on using the theory on GSBD functions which, initiated by DAL MAso [27], was developed over the last few years, see for example $[16,17,19,21-25,39,40,42,51]$. In fact, as a byproduct of our analysis, we introduce two new notions related to this function space: (1) a version of the space with functions attaining also the value infinity, and (2) a novel notion for convergence of rectifiable sets, which we call $\sigma_{\mathrm{sym}}^{p}$-convergence. Let us stress that in this work we exclusively consider a static setting. For evolutionary models, we mention the recent works $[36,43,44,55]$.

We now introduce the models under consideration in a slightly simplified way, restricting ourselves to three space dimensions. To describe material voids in elastically stressed solids, we consider the following functional defined on pairs of function-set (see [56]):

$$
F(u, E)=\int_{\Omega \backslash E} \mathbb{C} e(u): e(u) \mathrm{d} x+\int_{\Omega \cap \partial E} \varphi\left(v_{E}\right) \mathrm{d} \mathcal{H}^{2},
$$

where $E \subset \Omega$ represents the (sufficiently smooth) shape of voids within an elastic body with reference configuration $\Omega \subset \mathbb{R}^{3}$, and $u$ is an elastic displacement field. The first part of the functional represents the elastic energy depending on the linear strain $e(u):=\frac{1}{2}\left((\nabla u)^{\mathrm{T}}+\nabla u\right)$, where $\mathbb{C}$ denotes the fourth-order positive semi-definite tensor of elasticity coefficients. (In fact, we can incorporate more general elastic energies, see (2.2) below.) The surface energy depends on a (possibly anisotropic) density $\varphi$ evaluated at the outer normal $v_{E}$ to $E$. This setting is usually complemented with a volume constraint on the voids $E$ and nontrivial prescribed Dirichlet boundary conditions for $u$ on a part of $\partial \Omega$. We point out that the boundary conditions are the reason why the solid is elastically stressed. 
A variational model for epitaxially strained films can be regarded as a special case of (1.1) and corresponds to the situation where the material domain is the subgraph of an unknown nonnegative function $h$. More precisely, we assume that the material occupies the region

$$
\Omega_{h}^{+}:=\left\{x \in \omega \times \mathbb{R}: 0<x_{3}<h\left(x_{1}, x_{2}\right)\right\}
$$

for a given bounded function $h: \omega \rightarrow[0, \infty), \omega \subset \mathbb{R}^{2}$, whose graph represents the free profile of the film. We consider the energy

$$
G(u, h)=\int_{\Omega_{h}^{+}} \mathbb{C} e(u): e(u) \mathrm{d} x+\int_{\omega} \sqrt{1+\left|\nabla h\left(x_{1}, x_{2}\right)\right|^{2}} \mathrm{~d}\left(x_{1}, x_{2}\right) .
$$

Here, $u$ satisfies prescribed boundary data on $\omega \times\{0\}$ which corresponds to the interface between film and substrate. This Dirichlet boundary condition models the case of a film growing on an infinitely rigid substrate and is the reason for the film to be strained. We observe that (1.2) corresponds to (1.1) when $\varphi$ is the Euclidean norm, $\Omega=\omega \times(0, M)$ for some $M>0$ large enough, and $E=\Omega \backslash \Omega_{h}^{+}$.

Variants of the above models (1.1) and (1.2) have been studied by BRAIDES, Chambolle, and Solci [11] and by Chambolle and Solci [20], respectively, where the linearly elastic energy density $\mathbb{C} e(u): e(u)$ is replaced by an elastic energy satisfying a 2-growth (or $p$-growth, $p>1$ ) condition in the full gradient $\nabla u$ with quasiconvex integrands. These works are devoted to giving a sound mathematical formulation for determining equilibrium configurations. By means of variational methods and geometric measure theory, they study the relaxation of the functionals in terms of generalized functions of bounded variation $(G S B V$ ) which allows there to incorporate the possible roughness of the geometry of voids or films. The existence of minimizers for the relaxed functionals and the approximation of (the counterpart of) $G$ through a phase-field $\Gamma$-convergence result are addressed. In fact, the two articles were written almost simultaneously with many similarities in both the setting and the proof strategy.

Therefore, we prefer to present the extension of both works to the GSBD setting (that is, to three-dimensional linear elasticity) in a single work to allow for a comprehensive study of different applications. We now briefly discuss our main results.

(a) Relaxation of $F$ : We first note that, for fixed $E, F(\cdot, E)$ is weakly lower semicontinuous in $H^{1}$ and, for fixed $u, F(u, \cdot)$ can be regarded as a lower semicontinuous functional on sets of finite perimeter. The energy defined on pairs $(u, E)$, however, is not lower semicontinuous since, in a limiting process, the voids $E$ may collapse into a discontinuity of the displacement $u$. The relaxation has to take this phenomenon into account, in particular collapsed surfaces need to be counted twice in the relaxed energy. Provided that the surface density $\varphi$ is a norm in $\mathbb{R}^{3}$, we show that the relaxation takes the form (see Proposition 2.1)

$$
\bar{F}(u, E)=\int_{\Omega \backslash E} \mathbb{C} e(u): e(u) \mathrm{d} x+\int_{\Omega \cap \partial^{*} E} \varphi\left(v_{E}\right) \mathrm{d} \mathcal{H}^{2}+\int_{J_{u} \cap(\Omega \backslash E)^{1}} 2 \varphi\left(v_{u}\right) \mathrm{d} \mathcal{H}^{2},
$$


where $E$ is a set of finite perimeter with essential boundary $\partial^{*} E,(\Omega \backslash E)^{1}$ denotes the set of points of density 1 of $\Omega \backslash E$, and $u \in G S B D^{2}(\Omega)$. Here, $e(u)$ denotes the approximate symmetrized gradient of class $L^{2}\left(\Omega ; \mathbb{R}^{3 \times 3}\right)$ and $J_{u}$ is the jump set with corresponding measure-theoretical normal $v_{u}$. (We refer to Section 3 for the definition and the main properties of this function space. Later, we will also consider more general elastic energies and work with the space $\operatorname{GSBD}^{p}(\Omega), 1<p<\infty$, that is, $e(u) \in L^{p}\left(\Omega ; \mathbb{R}^{3 \times 3}\right)$.)

(b) Minimizer for $\bar{F}$ : In Theorem 2.2, we show that such a relaxation result can also be proved by imposing additionally a volume constraint on $E$ (which reflects mass conservation) and by prescribing boundary data for $u$. For this version of the relaxed functional, we prove the existence of minimizers, see Theorem 2.3.

(c) Relaxation of $G$ : For the model (1.2) describing epitaxially strained crystalline films, we show in Theorem 2.4 that the lower semicontinous envelope takes the form

$$
\bar{G}(u, h)=\int_{\Omega_{h}^{+}} \mathbb{C} e(u): e(u) \mathrm{d} x+\mathcal{H}^{2}\left(\Gamma_{h}\right)+2 \mathcal{H}^{2}(\Sigma),
$$

where $h \in B V(\omega ;[0, \infty))$ and $\Gamma_{h}$ denotes the (generalized) graph of $h$. Here, $u$ is again a $G S B D^{2}$-function and the set $\Sigma \subset \mathbb{R}^{3}$ is a "vertical" rectifiable set describing the discontinuity set of $u$ inside the subgraph $\Omega_{h}^{+}$. Similar to the last term in (1.3), this contribution has to be counted twice. We remark that in [35] the set $\Sigma$ is called "vertical cuts". Also here a volume constraint may be imposed.

(d) Minimizer for $\bar{G}$ : In Theorem 2.5, we show compactness for sequences with bounded $G$ energy. In particular, this implies existence of minimizers for $\bar{G}$ (under a volume constraint).

(e) Approximation for $\bar{G}$ : In Theorem 2.6, we finally prove a phase-field $\Gamma$ convergence approximation of $\bar{G}$. We remark that we can generalize the assumptions on the regularity of the Dirichlet datum. Whereas in [20, Theorem 5.1] the class $H^{1} \cap L^{\infty}$ was considered, we show that it indeed suffices to assume $H^{1}$-regularity.

We now provide some information on the proof strategy highlighting in particular the additional difficulties compared to $[11,20]$. Here, we will also explain why two new technical tools related to the space $G S B D$ have to be introduced.

(a) The proof of the lower inequality for the relaxation $\bar{F}$ is closely related to the analog in [11]: we use an approach by slicing, exploiting the lower inequality in one dimension, and employing a localization method. To prove the upper inequality, it is enough to combine the corresponding upper bound from [11] with a density result for $G S B D^{p}(p>1)$ functions [17], slightly adapted for our purposes, see Lemma 5.7.

(b) We point out that, in [11], the existence of minimizers was not addressed due to the lack of a compactness result. In this sense, our study also delivers a conceptionally new result without corresponding counterpart in [11]. The main difficulty lies in the fact that, for configurations with finite energy (1.3), small pieces of the body could be disconnected from the bulk part, either by the 
voids $E$ or by the jump set $J_{u}$. Thus, since there are no a priori bounds on the displacements, the function $u$ could attain arbitrarily large values on certain components, and this might rule out measure convergence for minimizing sequences. We remark that truncation methods, used to remedy this issue in scalar problems, are not applicable in the vectorial setting. This problem was solved only recently by general compactness results, both in the $G S B V^{p}$ and the $G S B D^{p}$ setting. The result [41] in $G S B V^{p}$ delivers a selection principle for minimizing sequences showing that one can always find at least one minimizing sequence converging in measure. With this, the existence of minimizers for the energies in [11] is immediate.

Our situation in linear elasticity, however, is more delicate, since a comparable result is not available in GSBD. In [19, Theorem 1.1], a compactness and lower semicontinuity result in $G S B D^{p}$ is derived relying on the idea that minimizing sequences may "converge to infinity" on a set of finite perimeter. In the present work, we refine this result by introducing a topology which induces this kind of nonstandard convergence. To this end, we need to define the new space $G S B D_{\infty}^{p}$ consisting of $G S B D^{p}$ functions which may also attain the value infinity. With these new techniques at hand, we can prove a general compactness result in $G S B D_{\infty}^{p}$ (see Theorem 5.9) which particularly implies the existence of minimizers for (1.3).

(c) Although the functional $G$ in (1.2) is a special case of $F$, the relaxation result is not an immediate consequence, due to the additional constraint that the domain is the subgraph of a function. Indeed, in the lower inequality, a further crucial step is needed in the description of the (variational) limit of $\partial \Omega_{h_{n}}$ when $h_{n} \rightarrow h$ in $L^{1}(\omega)$. In particular, the vertical set $\Sigma$ has to be identified, see (1.4).

This issue is connected to the problem of detecting all possible limits of jump sets $J_{u_{n}}$ of converging sequences $\left(u_{n}\right)_{n}$ of $G S B D^{p}$ functions. In the $G S B V^{p}$ setting, the notion of $\sigma^{p}$-convergence of sets is used, which has originally been developed by Dal Maso, FrancFort, AND ToAder [28] to study quasistatic crack evolution in nonlinear elasticity. (We refer also to the variant [47] which is independent of $p$.) In this work, we introduce an analogous notion in the $G S B D^{p}$ setting which we call $\sigma_{\mathrm{sym}}^{p}$-convergence. The definition is a bit more complicated compared to the $G S B V$ setting since it has to be formulated in the frame of $G S B D_{\infty}^{p}$ functions possibly attaining the value infinity. We believe that this notion may be of independent interest and is potentially helpful for studying other problems such as quasistatic crack evolution in linear elasticity [42]. We refer to Section 4 for the definition and properties of $\sigma_{\mathrm{sym}}^{p}$-convergence, as well as for a comparison to the corresponding notion in the $G S B V^{p}$ setting.

Showing the upper bound for the relaxation result is considerably more difficult than the analogous bound for $\bar{F}$. In fact, one has to guarantee that recovery sequences are made up by sets that are still subgraphs. We stress that this cannot be obtained by some general existence results, but is achieved through a very careful construction (pp. 29-38) that follows only partially the analogous one in [20]. We believe that the construction in [20] could indeed be improved by adopting an approach similar to ours, in order to take also some pathological situations into account. 
(d) To show the existence of minimizers of $G$, the delicate step is to prove that minimizing sequences have subsequences which converge (at least) in measure. In the $G S B V^{p}$ setting, this is simply obtained by applying a Poincaré inequality on vertical slices through the film. The same strategy cannot be pursued in $G S B D^{p}$, since by slicing in a certain direction not all components can be controlled. As a remedy, we proceed in two steps. We first use the novel compactness result in $G S B D_{\infty}^{p}$ to identify a limit which might attain the value infinity on a set of finite perimeter $G_{\infty}$. Then, a posteriori, we show that, actually, $G_{\infty}=\emptyset$; see Section 6.1 for details.

(e) For the phase-field approximation, we combine a variant of the construction in the upper inequality for $\bar{G}$ with the general strategy of the corresponding approximation result in [20]. The latter is slightly modified in order to proceed without $L^{\infty}$-bound on the displacements.

The paper is organized as follows: in Section 2, we introduce the setting of our two models on material voids in elastic solids and epitaxially strained films. Here, we also present our main relaxation, existence, and approximation results. Section 3 collects definition and main properties of the function space $G S B D^{p}$. In this section, we also define the space $G S B D_{\infty}^{p}$ and show basic properties. In Section 4 we introduce the novel notion of $\sigma_{\mathrm{sym}}^{p}$-convergence and prove a compactness result for sequences of rectifiable sets with bounded Hausdorff measure. Section 5 is devoted to the analysis of functionals defined on pairs of function-set. Finally, in Section 6 we investigate the model for epitaxially strained films and prove the relaxation, existence, and approximation results.

\section{Setting of the Problem and Statement of the Main Results}

In this section, we give the precise definitions of the two energy functionals and present the main relaxation, existence, and approximation results. In the following, $f: \mathbb{M}^{d \times d} \rightarrow[0, \infty)$ denotes a convex function satisfying the growth condition $(|\cdot|$ is the Frobenius norm on $\mathbb{M}^{d \times d}$ ):

$$
c_{1}\left|\zeta^{\mathrm{T}}+\zeta\right|^{p} \leqq f(\zeta) \leqq c_{2}\left(\left|\zeta^{\mathrm{T}}+\zeta\right|^{p}+1\right) \quad \text { for all } \zeta \in \mathbb{M}^{d \times d}
$$

and $f(0)=0$, for some $1<p<+\infty$. In particular, the convexity of $f$ and (2.1) imply that $f(\zeta)=f\left(\frac{1}{2}\left(\zeta^{\mathrm{T}}+\zeta\right)\right)$ for all $\zeta \in \mathbb{M}^{d \times d}$. For an open subset $\Omega \subset \mathbb{R}^{d}$, we will denote by $L^{0}\left(\Omega ; \mathbb{R}^{d}\right)$ the space of $\mathcal{L}^{d}$-measurable functions $v: \Omega \rightarrow \mathbb{R}^{d}$ endowed with the topology of the convergence in measure. We let $\mathfrak{M}(\Omega)$ be the family of all $\mathcal{L}^{d}$-measurable subsets of $\Omega$.

\subsection{Energies on Pairs Function-Set: Material Voids in Elastically Stressed Solids}

Let $\Omega \subset \mathbb{R}^{d}$ be a Lipschitz domain. We introduce an energy functional defined on pairs function-set. Given a norm $\varphi$ on $\mathbb{R}^{d}$ and $f: \mathbb{M}^{d \times d} \rightarrow[0, \infty)$, we let $F: L^{0}\left(\Omega ; \mathbb{R}^{d}\right) \times \mathfrak{M}(\Omega) \rightarrow \mathbb{R} \cup\{+\infty\}$ be defined by 


$$
F(u, E)= \begin{cases}\int_{\Omega \backslash E} f(e(u)) & \mathrm{d} x+\int_{\Omega \cap \partial E} \varphi\left(v_{E}\right) \mathrm{d} \mathcal{H}^{d-1} \\ & \text { if } \partial E \text { Lipschitz, }\left.u\right|_{\Omega \backslash \bar{E}} \in W^{1, p}\left(\Omega \backslash \bar{E} ; \mathbb{R}^{d}\right),\left.u\right|_{E}=0, \\ & \text { otherwise, }\end{cases}
$$

where $e(u):=\frac{1}{2}\left((\nabla u)^{\mathrm{T}}+\nabla u\right)$ denotes the symmetrized gradient, and $v_{E}$ the outer normal to $E$. We point out that the energy is determined by $E$ and the values of $u$ on $\Omega \backslash \bar{E}$. The condition $\left.u\right|_{E}=0$ is for definiteness only. We denote by $\bar{F}: L^{0}\left(\Omega ; \mathbb{R}^{d}\right) \times \mathfrak{M}(\Omega) \rightarrow \mathbb{R} \cup\{+\infty\}$ the lower semicontinuous envelope of the functional $F$ with respect to the convergence in measure for the functions and the $L^{1}(\Omega)$-convergence of characteristic functions of sets, that is,

$\bar{F}(u, E)=\inf \left\{\liminf _{n \rightarrow \infty} F\left(u_{n}, E_{n}\right): u_{n} \rightarrow u\right.$ in $L^{0}\left(\Omega ; \mathbb{R}^{d}\right)$ and $\chi_{E_{n}} \rightarrow \chi_{E}$ in $\left.L^{1}(\Omega)\right\}$.

(We observe that the convergence in $L^{0}\left(\Omega ; \mathbb{R}^{d}\right)$ is metrizable, so the sequential lower semicontinuous envelope coincides with the lower semicontinuous envelope with respect to this convergence.) In what follows, for any $s \in[0,1]$ and any $E \in \mathfrak{M}(\Omega), E^{s}$ denotes the set of points with density $s$ for $E$. By $\partial^{*} E$ we indicate its essential boundary, see [3, Definition 3.60]. For the definition of the space $\operatorname{GSBD}^{p}(\Omega), p>1$, we refer to Section 3 below. In particular, by $e(u)=\frac{1}{2}\left((\nabla u)^{\mathrm{T}}+\right.$ $\nabla u$ ) we denote the approximate symmetrized gradient, and by $J_{u}$ the jump set of $u$ with measure-theoretical normal $v_{u}$. We characterize $\bar{F}$ as follows:

Proposition 2.1. (Characterization of the lower semicontinuous envelope $\bar{F}$ ) Suppose that $f$ is convex and satisfies (2.1), and that $\varphi$ is a norm on $\mathbb{R}^{d}$. Then, it holds that

$\bar{F}(u, E)= \begin{cases}\int_{\Omega \backslash E} f(e(u)) \mathrm{d} x+\int_{\Omega \cap \partial^{*} E} & \varphi\left(v_{E}\right) \mathrm{d} \mathcal{H}^{d-1}+\int_{J_{u} \cap(\Omega \backslash E)^{1}} 2 \varphi\left(v_{u}\right) \mathrm{d} \mathcal{H}^{d-1} \\ & \text { if } u=u \chi_{E^{0}} \in G S B D^{p}(\Omega) \text { and } \mathcal{H}^{d-1}\left(\partial^{*} E\right)<+\infty \\ & \text { otherwise }\end{cases}$

Moreover, if $\mathcal{L}^{d}(E)>0$, then for any $(u, E) \in L^{0}\left(\Omega ; \mathbb{R}^{d}\right) \times \mathfrak{M}(\Omega)$ there exists a recovery sequence $\left(u_{n}, E_{n}\right)_{n} \subset L^{0}\left(\Omega ; \mathbb{R}^{d}\right) \times \mathfrak{M}(\Omega)$ such that $\mathcal{L}^{d}\left(E_{n}\right)=\mathcal{L}^{d}(E)$ for all $n \in \mathbb{N}$.

The last property shows that it is possible to incorporate a volume constraint on $E$ in the relaxation result. We now move on to consider a Dirichlet minimization problem associated to $F$. We will impose Dirichlet boundary data $u_{0} \in W^{1, p}\left(\mathbb{R}^{d} ; \mathbb{R}^{d}\right)$ on a subset $\partial_{D} \Omega \subset \partial \Omega$. For technical reasons, we suppose that $\partial \Omega=\partial_{D} \Omega \cup \partial_{N} \Omega \cup N$ with $\partial_{D} \Omega$ and $\partial_{N} \Omega$ relatively open, $\partial_{D} \Omega \cap \partial_{N} \Omega=\emptyset, \mathcal{H}^{d-1}(N)=0, \partial_{D} \Omega \neq \emptyset$, $\partial\left(\partial_{D} \Omega\right)=\partial\left(\partial_{N} \Omega\right)$, and that there exist a small $\bar{\delta}>0$ and $x_{0} \in \mathbb{R}^{d}$ such that for every $\delta \in(0, \bar{\delta})$, it holds that

$$
O_{\delta, x_{0}}\left(\partial_{D} \Omega\right) \subset \Omega
$$

where $O_{\delta, x_{0}}(x):=x_{0}+(1-\delta)\left(x-x_{0}\right)$. (These assumptions are related to Lemma 5.7 below.) In what follows, we denote by $\operatorname{tr}(u)$ the trace of $u$ on $\partial \Omega$ which 
is well defined for functions in $\operatorname{GSBD}^{p}(\Omega)$, see Section 3. In particular, it is well defined for functions $u$ considered in (2.2) satisfying $\left.u\right|_{\Omega \backslash \bar{E}} \in W^{1, p}\left(\Omega \backslash \bar{E} ; \mathbb{R}^{d}\right)$ and $\left.u\right|_{E}=0$. By $\nu_{\Omega}$ we denote the outer unit normal to $\partial \Omega$.

We now introduce a version of $F$ taking boundary data into account. Given $u_{0} \in W^{1, p}\left(\mathbb{R}^{d} ; \mathbb{R}^{d}\right)$, we set

$$
F_{\text {Dir }}(u, E)= \begin{cases}F(u, E)+\int_{\partial_{D} \Omega \cap \partial E} \varphi\left(v_{E}\right) \mathrm{d} \mathcal{H}^{d-1} & \text { if } \operatorname{tr}(u)=\operatorname{tr}\left(u_{0}\right) \text { on } \partial_{D} \Omega \backslash \bar{E} \\ +\infty & \text { otherwise. }\end{cases}
$$

Similarly to (2.3), we define the lower semicontinuous envelope $\bar{F}_{\text {Dir }}$ by

$$
\bar{F}_{\text {Dir }}(u, E)=\left\{\liminf _{n \rightarrow \infty} F_{\text {Dir }}\left(u_{n}, E_{n}\right): u_{n} \rightarrow u \text { in } L^{0}\left(\Omega ; \mathbb{R}^{d}\right) \text { and } \chi_{E_{n}} \rightarrow \chi_{E} \text { in } L^{1}(\Omega)\right\} .
$$

We have the following characterization:

Theorem 2.2. (Characterization of the lower semicontinuous envelope $\bar{F}_{\text {Dir }}$ ) Suppose that $f$ is convex and satisfies (2.1), that $\varphi$ is a norm on $\mathbb{R}^{d}$, and that (2.4) is satisfied. Then it holds that

$$
\bar{F}_{\operatorname{Dir}}(u, E)=\bar{F}(u, E)+\int_{\partial_{D} \Omega \cap \partial^{*} E} \varphi\left(v_{E}\right) \mathrm{d} \mathcal{H}^{d-1}+\int_{\left\{\operatorname{tr}(u) \neq \operatorname{tr}\left(u_{0}\right)\right\} \cap\left(\partial_{D} \Omega \backslash \partial^{*} E\right)} 2 \varphi\left(v_{\Omega}\right) \mathrm{d} \mathcal{H}^{d-1}
$$

Moreover, if $\mathcal{L}^{d}(E)>0$, then for any $(u, E) \in L^{0}\left(\Omega ; \mathbb{R}^{d}\right) \times \mathfrak{M}(\Omega)$ there exists a recovery sequence $\left(u_{n}, E_{n}\right)_{n} \subset L^{0}\left(\Omega ; \mathbb{R}^{d}\right) \times \mathfrak{M}(\Omega)$ such that $\mathcal{L}^{d}\left(E_{n}\right)=\mathcal{L}^{d}(E)$ for all $n \in \mathbb{N}$.

The proof of Proposition 2.1 and Theorem 2.2 will be given in Section 5.2. There, we provide also two slight generalizations (see Proposition 5.5 and Theorem 5.8), namely a relaxation with respect to a weaker convergence in a general space $G S B D_{\infty}^{p}$ (cf. (3.10)), where functions are allowed to attain the value infinity. We close this subsection with an existence result for $\bar{F}_{\text {Dir }}$, under a volume constraint for the voids.

Theorem 2.3. (Existence of minimizers for $\bar{F}_{\text {Dir }}$ ) Suppose that $f$ is convex and satisfies (2.1), and that $\varphi$ is a norm on $\mathbb{R}^{d}$. Let $m>0$. Then the minimization problem

$$
\inf \left\{\bar{F}_{\operatorname{Dir}}(u, E):(u, E) \in L^{0}\left(\Omega ; \mathbb{R}^{d}\right) \times \mathfrak{M}(\Omega), \mathcal{L}^{d}(E)=m\right\}
$$

admits solutions.

For the proof, we refer to Section 5.3; it relies on the lower semicontinuity of $\bar{F}_{\text {Dir }}$ and a compactness result in the general space $G S B D_{\infty}^{p}$ (cf. (3.10)), see Theorem 5.9. 


\subsection{Energies on Domains with a Subgraph Constraint: Epitaxially Strained Films}

We now consider the problem of displacement fields in a material domain which is the subgraph of an unknown nonnegative function $h$. Assuming that $h$ is defined on a Lipschitz domain $\omega \subset \mathbb{R}^{d-1}$, displacement fields $u$ will be defined on the subgraph

$$
\Omega_{h}^{+}:=\left\{x \in \omega \times \mathbb{R}: 0<x_{d}<h\left(x^{\prime}\right)\right\},
$$

where here and in the following we use the notation $x=\left(x^{\prime}, x_{d}\right)$ for $x \in \mathbb{R}^{d}$. To model Dirichlet boundary data at the flat surface $\omega \times\{0\}$, we will suppose that functions are extended to the set $\Omega_{h}:=\left\{x \in \omega \times \mathbb{R}:-1<x_{d}<h\left(x^{\prime}\right)\right\}$ and satisfy $u=u_{0}$ on $\omega \times(-1,0)$ for a given function $u_{0} \in W^{1, p}\left(\omega \times(-1,0) ; \mathbb{R}^{d}\right)$, $p>1$. In the application to epitaxially strained films, $u_{0}$ represents the substrate and $h$ represents the profile of the free surface of the film.

For convenience, we introduce the reference domain $\Omega:=\omega \times(-1, M+1)$ for $M>0$. We define the energy functional $G: L^{0}\left(\Omega ; \mathbb{R}^{d}\right) \times L^{1}(\omega ;[0, M]) \rightarrow$ $\mathbb{R} \cup\{+\infty\}$ by

$$
G(u, h)=\int_{\Omega_{h}^{+}} f(e(u(x))) \mathrm{d} x+\int_{\omega} \sqrt{1+\left|\nabla h\left(x^{\prime}\right)\right|^{2}} \mathrm{~d} x^{\prime}
$$

if $h \in C^{1}(\omega ;[0, M]),\left.u\right|_{\Omega_{h}} \in W^{1, p}\left(\Omega_{h} ; \mathbb{R}^{d}\right), u=0$ in $\Omega \backslash \Omega_{h}$, and $u=u_{0}$ in $\omega \times(-1,0)$, and $G(u, h)=+\infty$ otherwise. Here, $f: \mathbb{M}^{d \times d} \rightarrow[0, \infty)$ denotes a convex function satisfying $(2.1)$, and as before we set $e(u):=\frac{1}{2}\left((\nabla u)^{\mathrm{T}}+\nabla u\right)$. Notice that, in contrast to [9], we suppose that the functions $h$ are equibounded by a value $M$ : this is for technical reasons only and is indeed justified from a mechanical point of view since other effects come into play for very high crystal profiles.

We study the relaxation of $G$ with respect to the $L^{0}\left(\Omega ; \mathbb{R}^{d}\right) \times L^{1}(\omega ;[0, M])$ topology, that is, its lower semicontinuous envelope $\bar{G}: L^{0}\left(\Omega ; \mathbb{R}^{d}\right) \times L^{1}(\omega ;[0, M])$ $\rightarrow \mathbb{R} \cup\{+\infty\}$, defined as

$$
\bar{G}(u, h)=\inf \left\{\liminf _{n \rightarrow \infty} G\left(u_{n}, h_{n}\right): u_{n} \rightarrow u \operatorname{in} L^{0}\left(\Omega ; \mathbb{R}^{d}\right), h_{n} \rightarrow h \text { in } L^{1}(\omega)\right\} .
$$

We characterize $\bar{G}$ as follows, further assuming that the Lipschitz set $\omega \subset \mathbb{R}^{d-1}$ is uniformly star-shaped with respect to the origin, that is,

$$
t x \subset \omega \text { for all } t \in(0,1), x \in \partial \omega .
$$

Theorem 2.4. (Characterization of the lower semicontinuous envelope $\bar{G}$ ) Suppose that $f$ is convex satisfying (2.1) and that (2.9) holds. Then we have

$$
\bar{G}(u, h)=\left\{\begin{array}{l}
\int_{\Omega_{h}^{+}} f(e(u)) \mathrm{d} x+\mathcal{H}^{d-1}\left(\partial^{*} \Omega_{h} \cap \Omega\right)+2 \mathcal{H}^{d-1}\left(J_{u}^{\prime} \cap \Omega_{h}^{1}\right) \\
\quad \text { if } u=u \chi_{\Omega_{h}} \in G S B D^{p}(\Omega), u=u_{0} \text { in } \omega \times(-1,0), h \in B V(\omega ;[0, M]), \\
+\infty \quad \text { otherwise, }
\end{array}\right.
$$

where

$$
J_{u}^{\prime}:=\left\{\left(x^{\prime}, x_{d}+t\right): x \in J_{u}, t \geqq 0\right\} .
$$


The assumption (2.9) on $\omega$ is more general than the one considered in [20], where $\omega$ is assumed to be a torus. We point out, however, that both assumptions are only of technical nature and could be dropped at the expense of more elaborated estimates, see also [20]. The proof of this result will be given in Section 6.1.

We note that the functional $G$ could be considered with an additional volume constraint on the film, that is, $\mathcal{L}^{d}\left(\Omega_{h}^{+}\right)=\int_{\omega} h\left(x^{\prime}\right) \mathrm{d} x^{\prime}$ is fixed. An easy adaptation of the proof shows that the relaxed functional $\bar{G}$ is not changed under this constraint, see Remark 6.8 for details.

In Section 6.2, we further prove the following general compactness result, from which we deduce the existence of equilibrium configurations for epitaxially strained films:

Theorem 2.5. (Compactness for $\bar{G}$ ) Suppose that $f$ is convex and satisfies (2.1). For any $\left(u_{n}, h_{n}\right)_{n}$ with $\sup _{n} G\left(u_{n}, h_{n}\right)<+\infty$, there exist a subsequence (not relabeled) and functions $u \in G S B D^{p}(\Omega), h \in B V(\omega ;[0, M])$ with $u=u \chi \Omega_{h}$ and $u=u_{0}$ on $\omega \times(-1,0)$ such that

$$
\left(u_{n}, h_{n}\right) \rightarrow(u, h) \quad \text { in } \quad L^{0}\left(\Omega ; \mathbb{R}^{d}\right) \times L^{1}(\omega) .
$$

In particular, general properties of relaxation (see for example [26, Theorem 3.8]) imply that, given $0<m<M \mathcal{H}^{d-1}(\omega)$, the minimization problem

$$
\inf \left\{\bar{G}(u, h):(u, E) \in L^{0}\left(\Omega ; \mathbb{R}^{d}\right) \times L^{1}(\omega), \mathcal{L}^{d}\left(\Omega_{h}^{+}\right)=m\right\}
$$

admits solutions. Moreover, fixed $m$ and the volume constraint $\mathcal{L}^{d}\left(\Omega_{h}^{+}\right)=m$ for $G$ and $\bar{G}$, any cluster point for minimizing sequences of $G$ is a minimum point for $\bar{G}$.

Our final issue is a phase-field approximation of $\bar{G}$. The idea is to represent any subgraph $\Omega_{h}$ by a (regular) function $v$ which will be an approximation of the characteristic function $\chi_{\Omega_{h}}$ at a scale of order $\varepsilon$. Let $W:[0,1] \rightarrow[0, \infty)$ be continuous, with $W(1)=W(0)=0, W>0$ in $(0,1)$, and let $\left(\eta_{\varepsilon}\right)_{\varepsilon}$ with $\eta_{\varepsilon}>0$ and $\eta_{\varepsilon} \varepsilon^{1-p} \rightarrow 0$ as $\varepsilon \rightarrow 0$. Let $c_{W}:=\left(\int_{0}^{1} \sqrt{2 W(s)} \mathrm{d} s\right)^{-1}$. In the reference domain $\Omega=\omega \times(-1, M+1)$, we introduce the functionals

$$
G_{\varepsilon}(u, v):=\int_{\Omega}\left(\left(v^{2}+\eta_{\varepsilon}\right) f(e(u))+c_{W}\left(\frac{W(v)}{\varepsilon}+\frac{\varepsilon}{2}|\nabla v|^{2}\right)\right) \mathrm{d} x,
$$

if

$$
\begin{aligned}
& u \in W^{1, p}\left(\Omega ; \mathbb{R}^{d}\right), \quad u=u_{0} \text { in } \omega \times(-1,0), \\
& v \in H^{1}(\Omega ;[0,1]), \quad v=1 \text { in } \omega \times(-1,0), \\
& v=0 \text { in } \omega \times(M, M+1) \quad \partial_{d} v \leqq 0 \mathcal{L}^{d} \text {-a.e. in } \Omega,
\end{aligned}
$$

and $G_{\varepsilon}(u, v):=+\infty$ otherwise. The following phase-field approximation is the analog of [20, Theorem 5.1] in the frame of linear elasticity. We remark that here, differently from [20], we assume only $u_{0} \in W^{1, p}\left(\omega \times(-1,0) ; \mathbb{R}^{d}\right)$, and not necessarily $u_{0} \in L^{\infty}\left(\omega \times(-1,0) ; \mathbb{R}^{d}\right)$. For the proof we refer to Section 6.3. 
Theorem 2.6. Let $u_{0} \in W^{1, p}\left(\omega \times(-1,0) ; \mathbb{R}^{d}\right)$. For any decreasing sequence $\left(\varepsilon_{n}\right)_{n}$ of positive numbers converging to zero, the following hold:

(i) For any $\left(u_{n}, v_{n}\right)_{n}$ with $\sup _{n} G_{\varepsilon_{n}}\left(u_{n}, v_{n}\right)<+\infty$, there exist $u \in L^{0}\left(\Omega ; \mathbb{R}^{d}\right)$ and $h \in B V(\omega ;[0, M])$ such that, up to a subsequence, $u_{n} \rightarrow$ u a.e. in $\Omega$, $v_{n} \rightarrow \chi \Omega_{h}$ in $L^{1}(\Omega)$, and

$$
\bar{G}(u, h) \leqq \liminf _{n \rightarrow+\infty} G_{\varepsilon_{n}}\left(u_{n}, v_{n}\right) .
$$

(ii) For any $(u, h)$ with $\bar{G}(u, h)<+\infty$, there exists $\left(u_{n}, v_{n}\right)_{n}$ such that $u_{n} \rightarrow u$ a.e. in $\Omega, v_{n} \rightarrow \chi \Omega_{h}$ in $L^{1}(\Omega)$, and

$$
\limsup _{n \rightarrow \infty} G_{\varepsilon_{n}}\left(u_{n}, v_{n}\right)=\bar{G}(u, h) .
$$

\section{Preliminaries}

In this section, we recall the definition and main properties of the function space $G S B D^{p}$. Moreover, we introduce the space $G S B D_{\infty}^{p}$ of functions which may attain the value infinity.

\subsection{Notation}

For every $x \in \mathbb{R}^{d}$ and $\varrho>0$, let $B_{\varrho}(x) \subset \mathbb{R}^{d}$ be the open ball with center $x$ and radius $\varrho$. For $x, y \in \mathbb{R}^{d}$, we use the notation $x \cdot y$ for the scalar product and $|x|$ for the Euclidean norm. By $\mathbb{M}^{d \times d}$ and $\mathbb{M}_{\text {sym }}^{d \times d}$ we denote the set of matrices and symmetric matrices, respectively. We write $\chi_{E}$ for the indicator function of any $E \subset \mathbb{R}^{n}$, which is 1 on $E$ and 0 otherwise. If $E$ is a set of finite perimeter, we denote its essential boundary by $\partial^{*} E$, and by $E^{s}$ the set of points with density $s$ for $E$, see [3, Definition 3.60]. We indicate the minimum and maximum value between $a, b \in \mathbb{R}$ by $a \wedge b$ and $a \vee b$, respectively. The symmetric difference of two sets $A, B \subset \mathbb{R}^{d}$ is indicated by $A \triangle B$.

We denote by $\mathcal{L}^{d}$ and $\mathcal{H}^{k}$ the $d$-dimensional Lebesgue measure and the $k$ dimensional Hausdorff measure, respectively. For any locally compact subset $B \subset$ $\mathbb{R}^{d}$, (that is any point in $B$ has a neighborhood contained in a compact subset of $B$ ), the space of bounded $\mathbb{R}^{m}$-valued Radon measures on $B$ [respectively, the space of $\mathbb{R}^{m}$-valued Radon measures on $B$ ] is denoted by $\mathcal{M}_{b}\left(B ; \mathbb{R}^{m}\right)$ [resp., by $\left.\mathcal{M}\left(B ; \mathbb{R}^{m}\right)\right]$. If $m=1$, we write $\mathcal{M}_{b}(B)$ for $\mathcal{M}_{b}(B ; \mathbb{R}), \mathcal{M}(B)$ for $\mathcal{M}(B ; \mathbb{R})$, and $\mathcal{M}_{b}^{+}(B)$ for the subspace of positive measures of $\mathcal{M}_{b}(B)$. For every $\mu \in$ $\mathcal{M}_{b}\left(B ; \mathbb{R}^{m}\right)$, its total variation is denoted by $|\mu|(B)$. Given $\Omega \subset \mathbb{R}^{d}$ open, we use the notation $L^{0}\left(\Omega ; \mathbb{R}^{d}\right)$ for the space of $\mathcal{L}^{d}$-measurable functions $v: \Omega \rightarrow \mathbb{R}^{d}$.

Definition 3.1. Let $E \subset \mathbb{R}^{d}, v \in L^{0}\left(E ; \mathbb{R}^{m}\right)$, and $x \in \mathbb{R}^{d}$ such that

$$
\limsup _{\varrho \rightarrow 0^{+}} \frac{\mathcal{L}^{d}\left(E \cap B_{\varrho}(x)\right)}{\varrho^{d}}>0 .
$$


A vector $a \in \mathbb{R}^{d}$ is the approximate limit of $v$ as $y$ tends to $x$ if for every $\varepsilon>0$ it holds that

$$
\lim _{\varrho \rightarrow 0^{+}} \frac{\mathcal{L}^{d}\left(E \cap B_{\varrho}(x) \cap\{|v-a|>\varepsilon\}\right)}{\varrho^{d}}=0,
$$

and then we write

$$
\underset{y \rightarrow x}{\operatorname{ap}} \lim v(y)=a
$$

Definition 3.2. Let $U \subset \mathbb{R}^{d}$ be open and $v \in L^{0}\left(U ; \mathbb{R}^{m}\right)$. The approximate jump set $J_{v}$ is the set of points $x \in U$ for which there exist $a, b \in \mathbb{R}^{m}$, with $a \neq b$, and $v \in \mathbb{S}^{d-1}$ such that

$$
\underset{(y-x) \cdot v>0, y \rightarrow x}{\operatorname{ap} \lim } v(y)=a \text { and } \underset{(y-x) \cdot v<0, y \rightarrow x}{\operatorname{ap} \lim _{x \rightarrow 0}} v(y)=b .
$$

The triplet $(a, b, v)$ is uniquely determined up to a permutation of $(a, b)$ and a change of sign of $v$, and is denoted by $\left(v^{+}(x), v^{-}(x), v_{v}(x)\right)$. The jump of $v$ is the function defined by $[v](x):=v^{+}(x)-v^{-}(x)$ for every $x \in J_{v}$.

We note that $J_{v}$ is a Borel set with $\mathcal{L}^{d}\left(J_{v}\right)=0$, and that $[v]$ is a Borel function.

\section{2. $B V$ and BD Functions}

Let $U \subset \mathbb{R}^{d}$ be open. We say that a function $v \in L^{1}(U)$ is a function of bounded variation on $U$, and we write $v \in B V(U)$, if $\mathrm{D}_{i} v \in \mathcal{M}_{b}(U)$ for $i=1, \ldots, d$, where $\mathrm{D} v=\left(\mathrm{D}_{1} v, \ldots, \mathrm{D}_{d} v\right)$ is its distributional derivative. A vector-valued function $v: U \rightarrow \mathbb{R}^{m}$ is in $B V\left(U ; \mathbb{R}^{m}\right)$ if $v_{j} \in B V(U)$ for every $j=1, \ldots, m$. The space $B V_{\text {loc }}(U)$ is the space of $v \in L_{\text {loc }}^{1}(U)$ such that $\mathrm{D}_{i} v \in \mathcal{M}(U)$ for $i=1, \ldots, d$.

A function $v \in L^{1}\left(U ; \mathbb{R}^{d}\right)$ belongs to the space of functions of bounded deformation if the distribution $\mathrm{E} v:=\frac{1}{2}\left((\mathrm{D} v)^{\mathrm{T}}+\mathrm{D} v\right)$ belongs to $\mathcal{M}_{b}\left(U ; \mathbb{M}_{\text {sym }}^{d \times d}\right)$. It is well known (see [2,59]) that for $v \in B D(U), J_{v}$ is countably $\left(\mathcal{H}^{d-1}, d-1\right)$ rectifiable, and that

$$
\mathrm{E} v=\mathrm{E}^{a} v+\mathrm{E}^{c} v+\mathrm{E}^{j} v
$$

where $\mathrm{E}^{a} v$ is absolutely continuous with respect to $\mathcal{L}^{d}, \mathrm{E}^{c} v$ is singular with respect to $\mathcal{L}^{d}$ and such that $\left|\mathrm{E}^{c} v\right|(B)=0$ if $\mathcal{H}^{d-1}(B)<\infty$, while $\mathrm{E}^{j} v$ is concentrated on $J_{v}$. The density of $\mathrm{E}^{a} v$ with respect to $\mathcal{L}^{d}$ is denoted by $e(v)$.

The space $S B D(U)$ is the subspace of all functions $v \in B D(U)$ such that $\mathrm{E}^{c} v=0$. For $p \in(1, \infty)$, we define

$$
S B D^{p}(U):=\left\{v \in S B D(U): e(v) \in L^{p}\left(\Omega ; \mathbb{M}_{\mathrm{sym}}^{d \times d}\right), \mathcal{H}^{d-1}\left(J_{v}\right)<\infty\right\} .
$$

Analogous properties hold for $B V$, such as the countable rectifiability of the jump set and the decomposition of $\mathrm{D} v$. The spaces $S B V\left(U ; \mathbb{R}^{m}\right)$ and $S B V^{p}\left(U ; \mathbb{R}^{m}\right)$ are defined similarly, with $\nabla v$, the density of $\mathrm{D}^{a} v$, in place of $e(v)$. For a complete treatment of $B V, S B V$ functions and $B D, S B D$ functions, we refer to [3] and to $[2,6,59]$, respectively. 


\subsection{GBD Functions}

We now recall the definition and the main properties of the space $G B D$ of generalized functions of bounded deformation, introduced in [27], referring to that paper for a general treatment and more details. Since the definition of $G B D$ is given by slicing (differently from the definition of $G B V$, cf. [1,31]), we first need to introduce some notation. Fixed $\xi \in \mathbb{S}^{d-1}:=\left\{\xi \in \mathbb{R}^{d}:|\xi|=1\right\}$, we let

$\Pi^{\xi}:=\left\{y \in \mathbb{R}^{d}: y \cdot \xi=0\right\}, \quad B_{y}^{\xi}:=\{t \in \mathbb{R}: y+t \xi \in B\}$ for any $y \in \mathbb{R}^{d}$ and $B \subset \mathbb{R}^{d}$,

and for every function $v: B \rightarrow \mathbb{R}^{d}$ and $t \in B_{y}^{\xi}$ let

$$
v_{y}^{\xi}(t):=v(y+t \xi), \quad \widehat{v}_{y}^{\xi}(t):=v_{y}^{\xi}(t) \cdot \xi
$$

Definition 3.3. ([27]) Let $\Omega \subset \mathbb{R}^{d}$ be a bounded open set, and let $v \in L^{0}\left(\Omega ; \mathbb{R}^{d}\right)$. Then $v \in G B D(\Omega)$ if there exists $\lambda_{v} \in \mathcal{M}_{b}^{+}(\Omega)$ such that one of the following equivalent conditions holds true for every $\xi \in \mathbb{S}^{d-1}$ :

(a) for every $\tau \in C^{1}(\mathbb{R})$ with $-\frac{1}{2} \leqq \tau \leqq \frac{1}{2}$ and $0 \leqq \tau^{\prime} \leqq 1$, the partial derivative $\mathrm{D}_{\xi}(\tau(v \cdot \xi))=\mathrm{D}(\tau(v \cdot \xi)) \cdot \xi$ belongs to $\mathcal{M}_{b}(\Omega)$, and for every Borel set $B \subset \Omega$

$$
\left|\mathrm{D}_{\xi}(\tau(v \cdot \xi))\right|(B) \leqq \lambda_{v}(B)
$$

(b) $\widehat{v}_{y}^{\xi} \in B V_{\text {loc }}\left(\Omega_{y}^{\xi}\right)$ for $\mathcal{H}^{d-1}$-a.e. $y \in \Pi^{\xi}$, and for every Borel set $B \subset \Omega$

$$
\int_{\Pi^{\xi}}\left(\left|\mathrm{D} \widehat{v}_{y}^{\xi}\right|\left(B_{y}^{\xi} \backslash J_{\widehat{v}_{y}^{\xi}}^{1}\right)+\mathcal{H}^{0}\left(B_{y}^{\xi} \cap J_{\widehat{v}_{y}^{\xi}}^{1}\right)\right) \mathrm{d} \mathcal{H}^{d-1}(y) \leqq \lambda_{v}(B),
$$

where $J_{\widehat{u}_{y}^{\xi}}^{1}:=\left\{t \in J_{\widehat{u}_{y}^{\xi}}:\left|\left[\widehat{u}_{y}^{\xi}\right]\right|(t) \geqq 1\right\}$.

The function $v$ belongs to $G S B D(\Omega)$ if $v \in G B D(\Omega)$ and $\widehat{v}_{y}^{\xi} \in S B V_{\text {loc }}\left(\Omega_{y}^{\xi}\right)$ for every $\xi \in \mathbb{S}^{d-1}$ and for $\mathcal{H}^{d-1}$-a.e. $y \in \Pi^{\xi}$.

$G B D(\Omega)$ and $G S B D(\Omega)$ are vector spaces, as stated in [27, Remark 4.6], and one has the inclusions $B D(\Omega) \subset G B D(\Omega), S B D(\Omega) \subset G S B D(\Omega)$, which are in general strict (see [27, Remark 4.5 and Example 12.3]). Every $v \in G B D(\Omega)$ has an approximate symmetric gradient $e(v) \in L^{1}\left(\Omega ; \mathbb{M}_{\mathrm{sym}}^{d \times d}\right)$ such that for every $\xi \in \mathbb{S}^{d-1}$ and $\mathcal{H}^{d-1}$-a.e. $y \in \Pi^{\xi}$ it holds that

$$
e(v)(y+t \xi) \xi \cdot \xi=\left(\widehat{v}_{y}^{\xi}\right)^{\prime}(t) \text { for } \mathcal{L}^{1} \text {-a.e. } t \in \Omega_{y}^{\xi} .
$$

We recall also that by the area formula (cf. for example [57, (12.4)]; see [2, Theorem 4.10] and [27, Theorem 8.1]) it follows that for any $\xi \in \mathbb{S}^{d-1}$,

$$
\begin{aligned}
& \left(J_{v}^{\xi}\right)_{y}^{\xi}=J_{\widehat{v}_{y}^{\xi}} \text { for } \mathcal{H}^{d-1} \text {-a.e. } y \in \Pi^{\xi}, \text { where } J_{v}^{\xi}:=\left\{x \in J_{v}:[v](x) \cdot \xi \neq 0\right\}, \\
& \int_{\Pi^{\xi}} \mathcal{H}^{0}\left(J_{\widehat{v}_{y}^{\xi}}\right) \mathrm{d} \mathcal{H}^{d-1}(y)=\int_{J_{v}^{\xi}}\left|v_{v} \cdot \xi\right| \mathrm{d} \mathcal{H}^{d-1} .
\end{aligned}
$$


Moreover, it holds that

$$
\mathcal{H}^{d-1}\left(J_{v} \backslash J_{v}^{\xi}\right)=0 \quad \text { for } \mathcal{H}^{d-1} \text {-a.e. } \xi \in \mathbb{S}^{d-1} .
$$

Finally, if $\Omega$ has Lipschitz boundary, for each $v \in G B D(\Omega)$ the traces on $\partial \Omega$ are well defined in the sense that for $\mathcal{H}^{d-1}$-a.e. $x \in \partial \Omega$ there exists $\operatorname{tr}(v)(x) \in \mathbb{R}^{d}$ such that

$$
\operatorname{ap}_{y \rightarrow x, y \in \Omega} v(y)=\operatorname{tr}(v)(x) .
$$

For $1<p<\infty$, the space $\operatorname{GSBD}^{p}(\Omega)$ is defined by

$$
G S B D^{p}(\Omega):=\left\{u \in G S B D(\Omega): e(u) \in L^{p}\left(\Omega ; \mathbb{M}_{\text {sym }}^{d \times d}\right), \mathcal{H}^{d-1}\left(J_{u}\right)<\infty\right\} .
$$

We recall below two general density and compactness results in $G S B D^{p}$, from [17] and [19].

Theorem 3.4. (Density in $G S B D^{p}$ ) Let $\Omega \subset \mathbb{R}^{d}$ be an open, bounded set with finite perimeter and let $\partial \Omega$ be a $(d-1)$-rectifiable, $p>1, \psi(t)=t \wedge 1$, and $u \in G S B D^{p}(\Omega)$. Then there exist $u_{n} \in S B V^{p}\left(\Omega ; \mathbb{R}^{d}\right) \cap L^{\infty}\left(\Omega ; \mathbb{R}^{d}\right)$ such that each $J_{u_{n}}$ is closed in $\Omega$ and included in a finite union of closed connected pieces of $C^{1}$ hypersurfaces, $u_{n} \in W^{1, \infty}\left(\Omega \backslash J_{u_{n}} ; \mathbb{R}^{d}\right)$, and

$$
\begin{aligned}
\int_{\Omega} \psi\left(\left|u_{n}-u\right|\right) \mathrm{d} x & \rightarrow 0, \\
\left\|e\left(u_{n}\right)-e(u)\right\|_{L^{p}(\Omega)} & \rightarrow 0, \\
\mathcal{H}^{d-1}\left(J_{u_{n}} \triangle J_{u}\right) & \rightarrow 0 .
\end{aligned}
$$

We refer to [17, Theorem 1.1]. In contrast to [17], we use here the function $\psi(t):=t \wedge 1$ for simplicity. It is indeed easy to check that $[17,(1.1 \mathrm{e})]$ implies (3.6a).

Theorem 3.5. (GSBD ${ }^{p}$ compactness) Let $\Omega \subset \mathbb{R}$ be an open, bounded set, and let $\left(u_{n}\right)_{n} \subset G S B D^{p}(\Omega)$ be a sequence satisfying that

$$
\sup _{n \in \mathbb{N}}\left(\left\|e\left(u_{n}\right)\right\|_{L^{p}(\Omega)}+\mathcal{H}^{d-1}\left(J_{u_{n}}\right)\right)<+\infty .
$$

Then, there exists a subsequence, still denoted by $\left(u_{n}\right)_{n}$, such that the set $A:=$ $\left\{x \in \Omega:\left|u_{n}(x)\right| \rightarrow \infty\right\}$ has finite perimeter, and there exists $u \in \operatorname{GSBD}^{p}(\Omega)$ such that

(i) $u_{n} \rightarrow u \quad$ in $L^{0}\left(\Omega \backslash A ; \mathbb{R}^{d}\right)$,

(ii) $e\left(u_{n}\right) \rightarrow e(u)$ weakly in $L^{p}\left(\Omega \backslash A ; \mathbb{M}_{\mathrm{sym}}^{d \times d}\right)$,

(iii) $\liminf _{n \rightarrow \infty} \mathcal{H}^{d-1}\left(J_{u_{n}}\right) \geqq \mathcal{H}^{d-1}\left(J_{u} \cup\left(\partial^{*} A \cap \Omega\right)\right)$.

Moreover, for each $\Gamma \subset \Omega$ with $\mathcal{H}^{d-1}(\Gamma)<+\infty$, it holds that

$$
\liminf _{n \rightarrow \infty} \mathcal{H}^{d-1}\left(J_{u_{n}} \backslash \Gamma\right) \geqq \mathcal{H}^{d-1}\left(\left(J_{u} \cup\left(\partial^{*} A \cap \Omega\right)\right) \backslash \Gamma\right) .
$$

Proof. We refer to [19]. The additional statement (3.8) is proved, for example, in [42, Theorem 2.5].

Later, as a byproduct of our analysis, we will generalize the lower semicontinuity property (3.7)(iii) to anisotropic surface energies, see Corollary 5.6. 


\section{4. $G S B D_{\infty}^{p}$ Functions}

Inspired by the previous compactness result, we now introduce a space of $G S B D^{p}$ functions which may also attain a limit value $\infty$. Define $\overline{\mathbb{R}}^{d}:=\mathbb{R}^{d} \cup\{\infty\}$. The sum on $\overline{\mathbb{R}}^{d}$ is given by $a+\infty=\infty$ for any $a \in \overline{\mathbb{R}}^{d}$. There is a natural bijection between $\overline{\mathbb{R}}^{d}$ and $\mathbb{S}^{d}=\left\{\xi \in \mathbb{R}^{d+1}:|\xi|=1\right\}$ given by the stereographic projection of $\mathbb{S}^{d}$ to $\overline{\mathbb{R}}^{d}$ : for $\xi \neq e_{d+1}$, we define

$$
\phi(\xi)=\frac{1}{1-\xi_{d+1}}\left(\xi_{1}, \ldots, \xi_{d}\right),
$$

and let $\phi\left(e_{d+1}\right)=\infty$. By $\psi: \overline{\mathbb{R}}^{d} \rightarrow \mathbb{S}^{d}$ we denote the inverse. Note that

$$
d_{\overline{\mathbb{R}}^{d}}(x, y):=|\psi(x)-\psi(y)| \text { for } x, y \in \overline{\mathbb{R}}^{d}
$$

induces a bounded metric on $\overline{\mathbb{R}}^{d}$. We define

$$
\begin{array}{r}
G S B D_{\infty}^{p}(\Omega):=\left\{u \in L^{0}\left(\Omega ; \overline{\mathbb{R}}^{d}\right): A_{u}^{\infty}:=\{u=\infty\} \text { satisfies } \mathcal{H}^{d-1}\left(\partial^{*} A_{u}^{\infty}\right)<+\infty\right. \\
\left.\tilde{u}_{t}:=u \chi_{\Omega \backslash A_{u}^{\infty}}+t \chi_{A_{u}^{\infty}} \in G S B D^{p}(\Omega) \text { for all } t \in \mathbb{R}^{d}\right\} .
\end{array}
$$

Symbolically, we will also write

$$
u=u \chi \Omega \backslash A_{u}^{\infty}+\infty \chi_{A_{u}^{\infty}} .
$$

Moreover, for any $u \in G S B D_{\infty}^{p}(\Omega)$, we set $e(u)=0$ in $A_{u}^{\infty}$, and

$$
J_{u}=J_{u \chi_{\Omega \backslash A_{u}^{\infty}}} \cup\left(\partial^{*} A_{u}^{\infty} \cap \Omega\right) .
$$

In particular, we have that

$$
\begin{aligned}
e(u) & =e\left(\tilde{u}_{t}\right) \mathcal{L}^{d} \text {-a.e. on } \Omega \text { and } \\
J_{u} & =J_{\tilde{u}_{t}} \mathcal{H}^{d-1} \text {-a.e. for almost all } t \in \mathbb{R},
\end{aligned}
$$

where $\tilde{u}_{t}$ is the function from (3.10). Hereby, we also get a natural definition of a normal $v_{u}$ to the jump set $J_{u}$, and the slicing properties described in (3.3)-(3.5) still hold. Finally, we point out that all definitions are consistent with the usual ones if $u \in G S B D^{p}(\Omega)$, that is, if $A_{u}^{\infty}=\emptyset$. Since $G S B D^{p}(\Omega)$ is a vector space, we observe that the sum of two functions in $\operatorname{GSBD}_{\infty}^{p}(\Omega)$ lies again in this space.

A metric on $G S B D_{\infty}^{p}(\Omega)$ is given by

$$
\bar{d}(u, v):=\int_{\Omega} d_{\overline{\mathbb{R}}^{d}}(u(x), v(x)) \mathrm{d} x,
$$

where $d_{\overline{\mathbb{R}}^{d}}$ is the distance in (3.9). We now state compactness properties in $G S B D_{\infty}^{p}(\Omega)$. 
Lemma 3.6. (Compactness in $G S B D_{\infty}^{p}$ ) For $L>0$ and $\Gamma \subset \Omega$ with $\mathcal{H}^{d-1}(\Gamma)<$ $+\infty$, we introduce the sets

$$
\begin{aligned}
& X_{L}(\Omega)=\left\{v \in G S B D_{\infty}^{p}(\Omega): \mathcal{H}^{d-1}\left(J_{v}\right) \leqq L, \quad\|e(v)\|_{L^{p}(\Omega)} \leqq 1\right\}, \\
& X_{\Gamma}(\Omega)=\left\{v \in G S B D_{\infty}^{p}(\Omega): \mathcal{H}^{d-1}\left(J_{v} \backslash \Gamma\right)=0, \quad\|e(v)\|_{L^{p}(\Omega)} \leqq 1\right\} .
\end{aligned}
$$

Then the sets $X_{L}(\Omega), X_{\Gamma}(\Omega) \subset G S B D_{\infty}^{p}(\Omega)$ are compact with respect to the metric $\bar{d}$.

Proof. For $X_{L}(\Omega)$, the statement follows from Theorem 3.5 and the definitions (3.10)-(3.11): in fact, given a sequence $\left(u^{n}\right)_{n} \subset X_{L}(\Omega)$, we consider a sequence $\left(\tilde{u}_{t_{n}}^{n}\right)_{n} \subset G S B D^{p}(\Omega)$ as in (3.10), for suitable $\left(t_{n}\right)_{n} \subset \mathbb{R}^{d}$ with $\left|t_{n}\right| \rightarrow \infty$. This implies

$$
\bar{d}\left(u^{n}, \tilde{u}_{t_{n}}^{n}\right) \rightarrow 0 \text { as } n \rightarrow \infty .
$$

Then, by Theorem 3.5 there exists $v \in G S B D^{p}(\Omega)$ and $A=\left\{x \in \Omega:\left|\tilde{u}_{t_{n}}^{n}(x)\right| \rightarrow\right.$ $\infty\}$ such that $\tilde{u}_{t_{n}}^{n} \rightarrow v$ in $L^{0}\left(\Omega \backslash A ; \mathbb{R}^{d}\right)$. We define $u=v \chi_{\Omega \backslash A}+\infty \chi_{A} \in$ $G S B D_{\infty}^{p}(\Omega)$. By (3.7)(ii),(iii) and (3.11) we get that $u \in X_{L}(\Omega)$. We observe that $\bar{d}\left(\tilde{u}_{t_{n}}^{n}, u\right) \rightarrow 0$ and then by (3.15) also $\bar{d}\left(u^{n}, u\right) \rightarrow 0$.

The proof for the set $X_{\Gamma}(\Omega)$ is similar, where we additionally use (3.8) to ensure that $\mathcal{H}^{d-1}\left(J_{u} \backslash \Gamma\right)=0$.

In the next sections, we will use the following notation. We say that a sequence $\left(u_{n}\right)_{n} \subset G S B D_{\infty}^{p}(\Omega)$ converges weakly to $u \in G S B D_{\infty}^{p}(\Omega)$ if

$$
\sup _{n \in \mathbb{N}}\left(\left\|e\left(u_{n}\right)\right\|_{L^{p}(\Omega)}+\mathcal{H}^{d-1}\left(J_{u_{n}}\right)\right)<+\infty \text { and } \bar{d}\left(u_{n}, u\right) \rightarrow 0 \text { for } n \rightarrow \infty \text {. }
$$

We close this subsection by pointing out that a similar space has been introduced in [12], in the case of scalar valued functions attaining extended real values: the space $G B V_{*}\left(\mathbb{R}^{d}\right)$ was defined by $f: \mathbb{R}^{d} \rightarrow \mathbb{R} \cup\{ \pm \infty\} \in G B V_{*}\left(\mathbb{R}^{d}\right)$ if and only if $(-M \vee f) \wedge M \in B V_{\mathrm{loc}}\left(\mathbb{R}^{d}\right)$ for every $M>0$. In [12, Proposition 3.1] it is shown that $f \in G B V_{*}\left(\mathbb{R}^{d}\right)$ if and only if its epigraph is of locally finite perimeter in $\mathbb{R}^{d+1}$. Our definition is based on the structure of the set where functions attain infinite values, rather than employing (the analog of) truncations. In fact, the latter is not meaningful if one controls only symmetric gradients.

\section{The $\sigma_{\mathrm{sym}}^{p}$-Convergence of Sets}

This section is devoted to the introduction of a convergence of sets in the framework of $G S B D^{p}$ functions analogous to $\sigma^{p}$-convergence defined in [28] for the space $S B V^{p}$. This type of convergence of sets will be useful to study the lower limits in the relaxation results in Section 6.1 and the compactness properties in Section 6.2. We believe that this notion may be of independent interest and is potentially helpful to study also other problems such as quasistatic crack evolution. 
We start by recalling briefly the definition of $\sigma^{p}$-convergence in [28]: a sequence of sets $\left(\Gamma_{n}\right)_{n} \sigma^{p}$-converges to $\Gamma$ if (i) for any sequence $\left(u_{n}\right)_{n}$ converging to $u$ weakly in $S B V^{p}$ with $J_{u_{n}} \subset \Gamma_{n}$, it holds $J_{u} \subset \Gamma$ and (ii) there exists a $S B V^{p}$ function whose jump is $\Gamma$, which is approximated (in the sense of weak convergence in $S B V^{p}$ ) by $S B V^{p}$ functions with jump included in $\Gamma_{n}$. (Here, weak convergence in $S B V^{p}$ means that $\sup _{n}\left(\left\|u_{n}\right\|_{L^{\infty}}+\mathcal{H}^{d-1}\left(J_{u_{n}}\right)\right)<+\infty, \nabla u_{n} \rightarrow \nabla u$ in $L^{p}$, and $u_{n} \rightarrow u$ almost everywhere) For sequences of sets $\left(\Gamma_{n}\right)_{n}$ with $\sup _{n} \mathcal{H}^{d-1}\left(\Gamma_{n}\right)<$ $+\infty$, a compactness result with respect to $\sigma^{p}$-convergence is obtained by means of Ambrosio's compactness theorem [1]; see [28, Theorem 4.7] and [20, Theorem 3.3]. We refer to [28, Section 4.1] for a general motivation to consider such a kind of convergence.

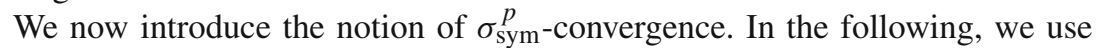
the notation $A \tilde{\subset} B$ if $\mathcal{H}^{d-1}(A \backslash B)=0$ and $A \tilde{=} B$ if $A \tilde{\subset} B$ and $B \tilde{\subset} A$. As before, by $(G)^{1}$ we denote the set of points with density 1 for $G \subset \mathbb{R}^{d}$. Recall also the definition and properties of $G S B D_{\infty}^{p}$ in Section 3.4, in particular (3.16).

Definition 4.1. ( $\sigma_{\text {sym }}^{p}$-convergence) Let $U \subset \mathbb{R}^{d}$ be open, let $U^{\prime} \supset U$ be open with $\mathcal{L}^{d}\left(U^{\prime} \backslash U\right)>0$, and let $p \in(1, \infty)$. We say that a sequence $\left(\Gamma_{n}\right)_{n} \subset \bar{U} \cap U^{\prime}$ with $\sup _{n \in \mathbb{N}} \mathcal{H}^{d-1}\left(\Gamma_{n}\right)<+\infty \sigma_{\text {sym }}^{p}$-converges to a pair $\left(\Gamma, G_{\infty}\right)$ satisfying $\Gamma \subset \bar{U} \cap U^{\prime}$ together with

$$
\mathcal{H}^{d-1}(\Gamma)<+\infty, \quad G_{\infty} \subset U, \quad \partial^{*} G_{\infty} \cap U^{\prime} \tilde{\subset} \Gamma, \quad \text { and } \quad \Gamma \cap\left(G_{\infty}\right)^{1}=\emptyset
$$

if it holds that:

(i) for any sequence $\left(v_{n}\right)_{n} \subset G S B D_{\infty}^{p}\left(U^{\prime}\right)$ with $J_{v_{n}} \tilde{\subset} \Gamma_{n}$ and $v_{n}=0$ in $U^{\prime} \backslash U$, if a subsequence $\left(v_{n_{k}}\right)_{k}$ converges weakly in $G S B D_{\infty}^{p}\left(U^{\prime}\right)$ to $v \in G S B D_{\infty}^{p}\left(U^{\prime}\right)$, then $\mathcal{L}^{d}\left(\{v=\infty\} \backslash G_{\infty}\right)=0$ and $J_{v} \backslash \Gamma \tilde{\subset}\left(G_{\infty}\right)^{1}$;

(ii) there exists a function $v \in G S B D_{\infty}^{p}\left(U^{\prime}\right)$ and a sequence $\left(v_{n}\right)_{n} \subset G S B D_{\infty}^{p}\left(U^{\prime}\right)$ converging weakly in $G S B D_{\infty}^{p}\left(U^{\prime}\right)$ to $v$ such that $J_{v_{n}} \tilde{\subset} \Gamma_{n}, v_{n}=0$ on $U^{\prime} \backslash U$ for all $n \in \mathbb{N}, J_{v} \tilde{=} \Gamma$, and $\{v=\infty\}=G_{\infty}$.

Our definition deviates from $\sigma^{p}$-convergence in the sense that, besides a limiting $(d-1)$-rectifiable set $\Gamma$, there exists also a set of finite perimeter $G_{\infty}$. Roughly speaking, in view of $\partial^{*} G_{\infty} \subset \Gamma \cup \partial U$, this set represents the parts which are completely disconnected by $\Gamma$ from the rest of the domain. The behavior of functions cannot be controlled there, that is, a sequence $\left(v_{n}\right)_{n}$ as in (i) may converge to infinity on this set or exhibit further cracks. In the framework of $G S B V^{p}$ functions in [28], it was possible to avoid such a phenomenon by working with truncations which allows to resort to $S B V^{p}$ functions with uniform $L^{\infty}$-bounds. In $G S B D$, however, this truncation technique is not available and we therefore need a more general definition involving the space $G S B D_{\infty}^{p}$ and a set of finite perimeter $G_{\infty}$.

Moreover, due to the presence of the set $G_{\infty}$, in contrast to the definition of $\sigma^{p}$ convergence, it is essential to control the functions in a set $U^{\prime} \backslash U$ : the assumptions $\mathcal{L}^{d}\left(U^{\prime} \backslash U\right)>0$ and $G_{\infty} \subset U$ are crucial since otherwise, if $U^{\prime}=U$, conditions (i) and (ii) would always be trivially satisfied with $G_{\infty}=U$ and $\Gamma=\emptyset$.

We briefly note that the pair $\left(\Gamma, G_{\infty}\right)$ is unique. In fact, if there were two different limits $\left(\Gamma^{1}, G_{\infty}^{1}\right)$ and $\left(\Gamma^{2}, G_{\infty}^{2}\right)$, we could choose functions $v^{1}$ and $v^{2}$ with 
$J_{v^{1}} \tilde{=} \Gamma^{1}, J_{v^{2}} \tilde{=} \Gamma^{2},\left\{v^{1}=\infty\right\}=G_{\infty}^{1}$, and $\left\{v^{2}=\infty\right\}=G_{\infty}^{2}$, as well as corresponding sequences $\left(v_{n}^{1}\right)_{n}$ and $\left(v_{n}^{2}\right)_{n}$ as in (ii). But then (i) implies $\Gamma^{1} \backslash \Gamma^{2} \tilde{\subset}\left(G_{\infty}^{2}\right)^{1}$, $\Gamma^{2} \backslash \Gamma^{1} \tilde{\subset}\left(G_{\infty}^{1}\right)^{1}$, as well as $G_{\infty}^{1} \subset G_{\infty}^{2}$ and $G_{\infty}^{2} \subset G_{\infty}^{1}$. As $\Gamma^{i} \cap\left(G_{\infty}^{i}\right)^{1}=\emptyset$ for $i=1,2$, this shows $\left(\Gamma^{1}, G_{\infty}^{1}\right)=\left(\Gamma^{2}, G_{\infty}^{2}\right)$. In a similar way, if a sequence $\left(\Gamma_{n}\right)_{n}$ $\sigma_{\text {sym }}^{p}$-converges to $\left(\Gamma, G_{\infty}\right)$, then every subsequence $\sigma_{\text {sym }}^{p}$-converges to the same limit.

Let us mention that, in our application in Section 6, the sets $\Gamma_{n}$ will be graphs of functions. In this setting, we will be able to ensure that $G_{\infty}=\emptyset$, see (6.3) below, and thus a simplification of Definition 4.1 only in terms of $\Gamma$ without $G_{\infty}$ is in principle possible. We believe, however, that the notion of $\sigma_{\mathrm{sym}}^{p}$-convergence may be of independent interest and is potentially helpful to study also other problems such as quasistatic crack evolution in linear elasticity [42], where $G_{\infty}=\varnothing$ cannot be expected. Therefore, we prefer to treat this more general definition here.

The main goal of this section is to prove the following compactness result for $\sigma_{\text {sym-convergence: }}^{p}$

Theorem 4.2. (Compactness of $\sigma_{\text {sym }}^{p}$-convergence) Let $U \subset \mathbb{R}^{d}$ be open, let $U^{\prime} \supset$ $U$ be open with $\mathcal{L}^{d}\left(U^{\prime} \backslash U\right)>0$, and let $p \in(1, \infty)$. Then, every sequence $\left(\Gamma_{n}\right)_{n} \subset$ $U$ with $\sup _{n} \mathcal{H}^{d-1}\left(\Gamma_{n}\right)<+\infty$ has a $\sigma_{\text {sym-convergent subsequence with limit }}^{p}$ $\left(\Gamma, G_{\infty}\right)$ satisfying $\mathcal{H}^{d-1}(\Gamma) \leqq \liminf _{n \rightarrow \infty} \mathcal{H}^{d-1}\left(\Gamma_{n}\right)$.

For the proof, we need the following two auxiliary results:

Lemma 4.3. Let $\left(v_{i}\right)_{i} \subset G S B D^{p}(\Omega)$ such that $\left\|e\left(v_{i}\right)\right\|_{L^{p}(\Omega)} \leqq 1$ for all $i$ and $\Gamma:=\bigcup_{i=1}^{\infty} J_{v_{i}}$ satisfies $\mathcal{H}^{d-1}(\Gamma)<+\infty$. Then there exist constants $c_{i}>0$, $i \in \mathbb{N}$, such that $\sum_{i=1}^{\infty} c_{i} \leqq 1$ and $v:=\sum_{i=1}^{\infty} c_{i} v_{i} \in G S B D^{p}(\Omega)$ satisfies $J_{v} \stackrel{\sim}{=} \Gamma$.

Lemma 4.4. Let $V \subset \mathbb{R}^{d}$ and suppose that two sequences $\left(u_{n}\right)_{n},\left(v_{n}\right)_{n} \in L^{0}\left(V ; \overline{\mathbb{R}}^{d}\right)$ satisfy $\left|u_{n}\right|,\left|v_{n}\right| \rightarrow \infty$ on $V$. Then for $\mathcal{L}^{1}$-a.e. $\theta \subset(0,1)$ it holds that

$$
\left|(1-\theta) u_{n}(x)+\theta v_{n}(x)\right| \rightarrow \infty \text { for a.e. } x \in V .
$$

We postpone the proof of the lemmas and proceed with the proof of Theorem 4.2.

Proof of Theorem 4.2. For $\Gamma \subset U$ with $\mathcal{H}^{d-1}(\Gamma)<+\infty$ we define

$$
X(\Gamma)=\left\{v \in G S B D_{\infty}^{p}\left(U^{\prime}\right): J_{v} \tilde{\subset} \Gamma, \quad\|e(v)\|_{L^{p}\left(U^{\prime}\right)} \leqq 1, \quad v=0 \text { on } U^{\prime} \backslash U\right\} .
$$

The set $X(\Gamma)$ is compact with respect to the metric $\bar{d}$ introduced in (3.13). This follows from Lemma 3.6 and the fact that $\left\{v \in L^{0}\left(U^{\prime} ; \overline{\mathbb{R}}^{d}\right): v=0\right.$ on $\left.U^{\prime} \backslash U\right\}$ is closed with respect to $\bar{d}$.

Since we treat any $v \in G S B D_{\infty}^{p}\left(U^{\prime}\right)$ as a constant function in the exceptional set $A_{v}^{\infty}$ (namely we have no jump and $e(v)=0$ therein, see (3.12)), we get that the convex combination of two $v, v^{\prime} \in X(\Gamma)$ is still in $X(\Gamma)$. (Recall that the sum on $\overline{\mathbb{R}}^{d}$ is given by $a+\infty=\infty$ for any $a \in \overline{\mathbb{R}}^{d}$.)

Step 1: Identification of a compact and convex subset. Consider $\left(\Gamma_{n}\right)_{n} \subset U$ with $\sup _{n} \mathcal{H}^{d-1}\left(\Gamma_{n}\right)<+\infty$. Fix $\delta>0$ small and define

$$
L:=\liminf _{n \rightarrow \infty} \mathcal{H}^{d-1}\left(\Gamma_{n}\right)+\delta .
$$


By (4.2) we have that, up to a subsequence (not relabeled), each $X\left(\Gamma_{n}\right)$ is contained in $X_{L}\left(U^{\prime}\right)$ defined in (3.14). Moreover, as noticed above, $X_{L}\left(U^{\prime}\right)$ and each $X\left(\Gamma_{n}\right)$ are compact with respect to $\bar{d}$. Since the class of non-empty compact subsets of a compact metric space $\left(M, d_{M}\right)$ is itself compact with respect to the Hausdorff distance induced by $d_{M}$, a subsequence (not relabeled) of $\left(X\left(\Gamma_{n}\right)\right)_{n}$ converges in the Hausdorff sense (with the Hausdorff distance induced by $\bar{d}$ ) to a compact set $K \subset X_{L}\left(U^{\prime}\right)$.

We first observe that the function identical to zero lies in $K$. We now show that $K$ is convex. Choose $u, v \in K$ and $\theta \in(0,1)$. We need to check that $w:=$ $(1-\theta) u+\theta v \in K$. Observe that $A_{w}^{\infty}=A_{u}^{\infty} \cup A_{v}^{\infty}$, where $A_{u}^{\infty}, A_{v}^{\infty}$, and $A_{w}^{\infty}$ are the exceptional sets given in (3.10). There exist sequences $\left(u_{n}\right)_{n}$ and $\left(v_{n}\right)_{n}$ with $u_{n}, v_{n} \in X\left(\Gamma_{n}\right)$ such that $\bar{d}\left(u_{n}, u\right) \rightarrow 0$ and $\bar{d}\left(v_{n}, v\right) \rightarrow 0$. In particular, note that $\left|u_{n}\right| \rightarrow \infty$ on $A_{u}^{\infty}$ and $\left|v_{n}\right| \rightarrow \infty$ on $A_{v}^{\infty}$. By Lemma 4.4 and a diagonal argument we can choose $\left(\theta_{n}\right)_{n} \subset(0,1)$ with $\theta_{n} \rightarrow \theta$ such that $w_{n}:=\left(1-\theta_{n}\right) u_{n}+\theta_{n} v_{n}$ satisfies $\left|w_{n}\right| \rightarrow \infty$ on $A_{u}^{\infty} \cap A_{v}^{\infty}$. As clearly $\left|w_{n}\right| \rightarrow \infty$ on $A_{u}^{\infty} \triangle A_{v}^{\infty}$ and $(1-$ $\left.\theta_{n}\right) u_{n}+\theta_{n} v_{n} \rightarrow(1-\theta) u+\theta v$ in measure on $U^{\prime} \backslash\left(A_{u}^{\infty} \cup A_{v}^{\infty}\right)$, we get $\bar{d}\left(w_{n}, w\right) \rightarrow 0$. Since $X\left(\Gamma_{n}\right)$ is convex, there holds $w_{n} \in X\left(\Gamma_{n}\right)$. Then $\bar{d}\left(w_{n}, w\right) \rightarrow 0$ implies $w \in K$, as desired.

Step 2: Choice of dense subset. Since $K$ is compact with respect to the metric $\bar{d}$ (so, in particular, $K$ is separable), we can choose a countable set $\left(y_{i}\right)_{i} \subset G S B D_{\infty}^{p}\left(U^{\prime}\right)$ with $y_{i}=0$ on $U^{\prime} \backslash U$ which is $\bar{d}$-dense in $K$. We now show that this countable set can be chosen with the additional property

$$
\mathcal{L}^{d}\left(A_{v}^{\infty} \backslash \bigcup_{i} A_{y_{i}}^{\infty}\right)=0 \quad \text { for all } v \in K
$$

where we again denote by $A_{y_{i}}^{\infty}$ and $A_{v}^{\infty}$ the sets where the functions attain the value $\infty$. In fact, fix an arbitrary countable and $\bar{d}$-dense set $\left(y_{i}\right)_{i}$ in $K$, and let $\eta>0$. After adding a finite number (smaller than $\mathcal{L}^{d}(U) / \eta$ ) of functions of $K$ to this collection, we obtain a countable $\bar{d}$-dense family $\left(y_{i}^{\eta}\right)_{i}$ such that

$$
\mathcal{L}^{d}\left(A_{v}^{\infty} \backslash \bigcup_{i} A_{y_{i}^{\eta}}^{\infty}\right) \leqq \eta \quad \text { for all } v \in K
$$

Then, we obtain the desired countable set by taking the union of $\left(y_{i}^{1 / k}\right)_{i}$ for $k \in \mathbb{N}$. Step 3: Definition of $\Gamma$ and $G_{\infty}$. Fix $v, v^{\prime} \in K$. Since $\left\{x \in J_{v} \backslash \partial^{*} A_{v}^{\infty}:[v](x)=\right.$ $t$ \} has negligible $\mathcal{H}^{d-1}$-measure up to a countable set of points $t$, we find some $\theta \in(0,1)$ such that $w:=\theta v+(1-\theta) v^{\prime}$ satisfies

$$
J_{w} \tilde{\subset} J_{v} \cup J_{v^{\prime}}, \quad\left(J_{v} \cup J_{v^{\prime}}\right) \backslash J_{w} \tilde{\subset}\left(A_{v}^{\infty} \cup A_{v^{\prime}}^{\infty}\right)^{1} .
$$

Here, we particularly point out that $\{w=\infty\}=A_{v}^{\infty} \cup A_{v^{\prime}}^{\infty}$ and that $\partial^{*}\left(A_{v}^{\infty} \cup A_{v^{\prime}}^{\infty}\right) \cap$ $U^{\prime} \tilde{\subset} J_{w}$ by (3.11). Note that $w \in K$ since $K$ is convex. Since $w \in K \subset X_{L}\left(U^{\prime}\right)$, (4.4) implies that

$$
\begin{aligned}
& \mathcal{H}^{d-1}\left(\left(J_{v} \cup J_{v^{\prime}}\right) \backslash\left(A_{v}^{\infty} \cup A_{v^{\prime}}^{\infty}\right)^{1}\right) \leqq \mathcal{H}^{d-1}\left(J_{w}\right) \leqq L, \\
& \mathcal{H}^{d-1}\left(\partial^{*}\left(A_{v}^{\infty} \cup A_{v^{\prime}}^{\infty}\right) \cap U^{\prime}\right) \leqq \mathcal{H}^{d-1}\left(J_{w}\right) \leqq L
\end{aligned}
$$


Let $\left(y_{i}\right)_{i} \subset G S B D_{\infty}^{p}\left(U^{\prime}\right)$ with $y_{i}=0$ on $U^{\prime} \backslash U$ be the countable and $\bar{d}$-dense subset of $K$ satisfying (4.3) that we defined in Step 2. By the above convexity argument, we find

$$
\mathcal{H}^{d-1}\left(\bigcup_{i=1}^{k} J_{y_{i}} \backslash\left(\bigcup_{i=1}^{k} A_{i}\right)^{1}\right) \leqq L, \quad \mathcal{H}^{d-1}\left(\partial^{*}\left(\bigcup_{i=1}^{k} A_{i}\right) \cap U^{\prime}\right) \leqq L
$$

for all $k \in \mathbb{N}$, where

$$
A_{i}:=A_{y_{i}}^{\infty}=\left\{y_{i}=\infty\right\}
$$

We define

$$
G_{\infty}:=\bigcup_{i=1}^{\infty} A_{i}
$$

By passing to the limit $k \rightarrow \infty$ in (4.5), we get $\mathcal{H}^{d-1}\left(\partial^{*} G_{\infty} \cap U^{\prime}\right) \leqq L$ and $\mathcal{H}^{d-1}\left(\bigcup_{i=1}^{k} J_{y_{i}} \backslash\left(G_{\infty}\right)^{1}\right) \leqq L$ for all $k \in \mathbb{N}$. Passing again to the limit $k \rightarrow \infty$, and setting

$$
\Gamma:=\bigcup_{i=1}^{\infty} J_{y_{i}} \backslash\left(G_{\infty}\right)^{1}
$$

we get $\mathcal{H}^{d-1}(\Gamma) \leqq L$. Notice that $\Gamma \cap\left(G_{\infty}\right)^{1}=\emptyset$ by definition. Moreover, the fact that $y_{i}=0$ on $U^{\prime} \backslash U$ for all $i \in \mathbb{N}$ implies both that $G_{\infty} \subset U$ and that $\Gamma \subset \bar{U} \cap U^{\prime}$. By (4.2) and the arbitrariness of $\delta$ we get $\mathcal{H}^{d-1}(\Gamma) \leqq \liminf _{n \rightarrow \infty} \mathcal{H}^{d-1}\left(\Gamma_{n}\right)$. Since $\partial^{*} A_{i} \cap U^{\prime} \tilde{\subset} J_{y_{i}}$ for all $i \in \mathbb{N}$ by (3.11), we also get $\Gamma \tilde{\supset} \partial^{*} G_{\infty} \cap U^{\prime}$. Thus, (4.1) is satisfied.

We now claim that for each $v \in K$ it holds that

$$
\mathcal{L}^{d}\left(\{v=\infty\} \backslash G_{\infty}\right)=0 \quad \text { and } \quad J_{v} \backslash \Gamma \tilde{\subset}\left(G_{\infty}\right)^{1} .
$$

Indeed, the first property follows from (4.3) and (4.6). To see the second, we note that, for any fixed $v \in K$, there is a sequence $\left(y_{k}\right)_{k}=\left(y_{i_{k}}\right)_{k}$ with $\bar{d}\left(y_{k}, v\right) \rightarrow 0$, by the density of $\left(y_{i}\right)_{i}$. Consider the functions $\tilde{v}_{k}:=y_{k}\left(1-\chi_{G_{\infty}}\right)$ that $\bar{d}$-converge to $\tilde{v}:=v\left(1-\chi_{G_{\infty}}\right)$ : since $J_{\tilde{v}_{k}} \tilde{\subset} \Gamma$ for any $k$ (we employ (4.7) and that $\partial^{*} G_{\infty} \cap U^{\prime} \tilde{\subset} \Gamma$ ), the fact that $X(\Gamma)$ is closed gives that $J_{\tilde{v}} \tilde{\subset} \Gamma$. This implies (4.8).

Step 4: Proof of properties ( $i$ ) and (ii). We first show (i). Given a sequence $\left(v_{n}\right)_{n} \subset$ $G S B D_{\infty}^{p}\left(U^{\prime}\right)$ with $J_{v_{n}} \tilde{\subset} \Gamma_{n}$ and $v_{n}=0$ on $U^{\prime} \backslash U$, and a subsequence $\left(v_{n_{k}}\right)_{k}$ that converges weakly in $G S B D_{\infty}^{p}\left(U^{\prime}\right)$ to $v$, we clearly get $v \in K$ by Hausdorff convergence of $X\left(\Gamma_{n}\right) \rightarrow K$. (More precisely, consider $\lambda v_{n_{k}}$ and $\lambda v$ for $\lambda>0$ such that $\left\|e\left(\lambda v_{n_{k}}\right)\right\|_{L^{p}\left(U^{\prime}\right)} \leqq 1$ for all $k$.) By (4.8), this implies $\mathcal{L}^{d}\left(\{v=\infty\} \backslash G_{\infty}\right)=0$ and $J_{v} \backslash \Gamma \tilde{\subset}\left(G_{\infty}\right)^{1}$. This shows (i).

We now address (ii). Recalling the choice of the sequence $\left(y_{i}\right)_{i} \subset K$, for each $i \in \mathbb{N}$, we choose $\tilde{y}_{i}=y_{i} \chi_{U^{\prime} \backslash G_{\infty}}+t_{i} \chi_{G_{\infty}} \in G S B D^{p}\left(U^{\prime}\right)$ for some $t_{i} \in \mathbb{R}^{d}$ such that $J_{\tilde{y}_{i}} \stackrel{\sim}{=} J_{y_{i}} \backslash\left(G_{\infty}\right)^{1}$. (Almost every $t_{i}$ works. Note that the function indeed lies in $G S B D^{p}(U)$, see (3.10) and (4.6).) In view of (4.7), we also observe that $\bigcup_{i} J_{\tilde{y}_{i}}=\Gamma$.

By Lemma 4.3 (recall $\left(y_{i}\right)_{i} \subset K \subset X_{L}(\Omega)$ ) we get a function $\tilde{v}=\sum_{i=1}^{\infty} c_{i} \tilde{y}_{i} \in$ $G S B D^{p}\left(U^{\prime}\right)$ such that $J_{\tilde{v}} \stackrel{\sim}{=} \Gamma$, where $\sum_{i=1}^{\infty} c_{i} \leqq 1$. We also define $v=\tilde{v} \chi_{U^{\prime} \backslash G_{\infty}}+$ 
$\infty \chi_{G_{\infty}} \in G S B D_{\infty}^{p}\left(U^{\prime}\right)$. Note that $\{v=\infty\}=G_{\infty}$ and $J_{v} \tilde{=} \Gamma$ since $\Gamma \cap\left(G_{\infty}\right)^{1}=$ $\emptyset$ and $\partial^{*} G_{\infty} \cap U^{\prime} \subset \Gamma$. Then by the convexity of $K$, we find $z_{k}:=\sum_{i=1}^{k} c_{i} y_{i} \in K$. (Here we also use that the function identical to zero lies in $K$.) As $G_{\infty}=\bigcup_{i=1}^{\infty} A_{i}$, we obtain $\bar{d}\left(z_{k}, v\right) \rightarrow 0$ for $k \rightarrow \infty$. Thus, also $v \in K$ since $K$ is compact. As $X\left(\Gamma_{n}\right)$ converges to $K$ in Hausdorff convergence, we find a sequence $\left(v_{n}\right)_{n} \subset$ $G S B D_{\infty}^{p}\left(U^{\prime}\right)$ with $J_{v_{n}} \tilde{\subset} \Gamma_{n}, v_{n}=0$ on $U^{\prime} \backslash U$, and $\bar{d}\left(v_{n}, v\right) \rightarrow 0$. This shows (ii).

Next, we prove Lemma 4.3. To this end, we will need the following measuretheoretical result (see [38, Lemma 4.1, 4.2] and note that the statement in fact holds in arbitrary space dimensions for measurable functions.):

Lemma 4.5. Let $\Omega \subset \mathbb{R}^{d}$ with $\mathcal{L}^{d}(\Omega)<\infty$, and $N \in \mathbb{N}$. Then for every sequence $\left(u_{n}\right)_{n} \subset L^{0}\left(\Omega ; \mathbb{R}^{N}\right)$ with

$$
\mathcal{L}^{d}\left(\bigcap_{n \in \mathbb{N}} \bigcup_{m \geqq n}\left\{\left|u_{m}-u_{n}\right|>1\right\}\right)=0,
$$

there exists a subsequence (not relabeled) and an increasing concave function $\psi:[0, \infty) \rightarrow[0, \infty)$ with $\lim _{t \rightarrow \infty} \psi(t)=+\infty$ such that

$$
\sup _{n \geqq 1} \int_{\Omega} \psi\left(\left|u_{n}\right|\right) \mathrm{d} x<+\infty .
$$

Proof of Lemma 4.3. Let $\left(v_{i}\right)_{i} \subset G S B D^{p}(\Omega)$ be given satisfying the assumptions of the lemma. First, choose $0<d_{i}<2^{-i}$ such that

$$
\mathcal{L}^{d}\left(\left\{\left|v_{i}\right| \geqq \frac{1}{2^{i} d_{i}}\right\}\right) \leqq 2^{-i}, \quad \mathcal{H}^{d-1}\left(\left\{x \in J_{v_{i}}:\left|\left[v_{i}\right](x)\right| \geqq \frac{1}{d_{i}}\right\}\right) \leqq 2^{-i} .
$$

Our goal is to select constants $c_{i} \in\left(0, d_{i}\right)$ such that the function $v:=\sum_{i=1}^{\infty} c_{i} v_{i}$ lies in $\operatorname{GSBD}^{p}(\Omega)$ and satisfies $J_{v} \tilde{=} \Gamma:=\bigcup_{i=1}^{\infty} J_{v_{i}}$. We proceed in two steps: we first show that for each choice $c_{i} \in\left(0, d_{i}\right)$ the function $v=\sum_{i=1}^{\infty} c_{i} v_{i}$ lies indeed in $G S B D^{p}(\Omega)$ (Step 1). Afterwards, we prove that for a specific choice it holds that $J_{v} \stackrel{\sim}{=} \Gamma$.

Step 1. Given $c_{i} \in\left(0, d_{i}\right)$, we define $u_{k}=\sum_{i=1}^{k} c_{i} v_{i}$. Fix $m \geqq n+1$. We observe that

$$
\begin{aligned}
\left\{\left|u_{m}-u_{n}\right|>1\right\} & =\left\{\left|\sum_{i=n+1}^{m} c_{i} v_{i}\right|>1\right\} \subset \bigcup_{i=n+1}^{m}\left\{\left|c_{i} v_{i}\right| \geqq 2^{-i}\right\} \\
& \subset \bigcup_{i=n+1}^{m}\left\{\left|v_{i}\right| \geqq \frac{1}{2^{i} d_{i}}\right\} .
\end{aligned}
$$

By passing to the limit $m \rightarrow \infty$ and by using (4.10) we get

$$
\begin{aligned}
\mathcal{L}^{d}\left(\bigcup_{m \geqq n}\left\{\left|u_{m}-u_{n}\right|>1\right\}\right) & \leqq \sum_{i=n+1}^{\infty} \mathcal{L}^{d}\left(\left\{\left|v_{i}\right| \geqq \frac{1}{2^{i} d_{i}}\right\}\right) \\
& \leqq \sum_{i=n+1}^{\infty} 2^{-i}=2^{-n} .
\end{aligned}
$$


This shows that the sequence $\left(u_{k}\right)_{k}$ satisfies (4.9), and therefore there exist a subsequence (not relabeled) and an increasing continuous function $\psi:[0, \infty) \rightarrow[0, \infty)$ with $\lim _{t \rightarrow \infty} \psi(t)=+\infty$ such that $\sup _{k} \geqq 1 \int_{\Omega} \psi\left(\left|u_{k}\right|\right) \mathrm{d} x<+\infty$. Recalling also that $\left\|e\left(v_{i}\right)\right\|_{L^{p}(\Omega)} \leqq 1$ for all $i$ and $\mathcal{H}^{d-1}(\Gamma)<+\infty$, we are now in the position to apply the $G S B D^{p}$-compactness result [27, Theorem 11.3] (alternatively, one could apply Theorem 3.5 and observe that the limit $v$ satisfies $\left.\mathcal{L}^{d}(\{v=\infty\})=0\right)$, to get that the function $v=\sum_{i=1}^{\infty} c_{i} v_{i}$ lies in $\operatorname{GSBD}^{p}(\Omega)$. For later purposes, we note that by (3.8) (which holds also in addition to [27, Theorem 11.3]) we obtain

$$
J_{v} \tilde{\subset} \bigcup_{i=1}^{\infty} J_{v_{i}}=\Gamma
$$

This concludes Step 1 of the proof.

Step 2. We define the constants $c_{i} \in\left(0, d_{i}\right)$ iteratively by following the arguments in [28, Lemma 4.5]. Suppose that $\left(c_{i}\right)_{i=1}^{k}$, and a decreasing sequence $\left(\varepsilon_{i}\right)_{i=1}^{k} \subset(0,1)$ have been chosen such that the functions $u_{j}=\sum_{i=1}^{j} c_{i} v_{i}$, $1 \leqq j \leqq k$, satisfy

$$
\begin{aligned}
& \text { (i) } J_{u_{j}} \stackrel{\sim}{=} \bigcup_{i=1}^{j} J_{v_{i}}, \\
& \text { (ii) } \mathcal{H}^{d-1}\left(\left\{x \in J_{u_{j}}:\left|\left[u_{j}\right](x)\right| \leqq \varepsilon_{j}\right\}\right) \leqq 2^{-j},
\end{aligned}
$$

and, for $2 \leqq j \leqq k$, it holds that

$$
c_{j} \leqq \varepsilon_{j-1} d_{j} 2^{-j-1} .
$$

(Note that in the first step we can simply set $c_{1}=1 / 4$ and $0<\varepsilon_{1}<1$ such that (4.12)(ii) holds.)

We pass to the step $k+1$ as follows. Note that there is a set $N_{0} \subset \mathbb{R}$ of negligible measure such that for all $t \in \mathbb{R} \backslash N_{0}$ there holds $J_{u_{k}+t v_{k+1}} \stackrel{=}{=} J_{u_{k}} \cup J_{v_{k+1}}$. We choose $c_{k+1} \in \mathbb{R} \backslash N_{0}$ such that additionally $c_{k+1} \leqq \varepsilon_{k} d_{k+1} 2^{-k-2}$. Then (4.12)(i) and (4.13) hold. We can then choose $\varepsilon_{k+1} \leqq \varepsilon_{k}$ such that also (4.12)(ii) is satisfied.

We proceed in this way for all $k \in \mathbb{N}$. Let us now introduce the sets

$$
E_{k}=\bigcup_{m \geqq k}\left\{x \in J_{u_{m}}:\left|\left[u_{m}\right](x)\right| \leqq \varepsilon_{m}\right\}, \quad F_{k}=\bigcup_{m \geqq k}\left\{x \in J_{v_{m}}:\left|\left[v_{m}\right](x)\right|>1 / d_{m}\right\} .
$$

Note by (4.10) and (4.12)(ii) that

$$
\mathcal{H}^{d-1}\left(E_{k} \cup F_{k}\right) \leqq 2 \sum_{m \geqq k} 2^{-m}=2^{2-k} .
$$

We now show that for all $k \in \mathbb{N}$ it holds that

$$
J_{u_{k}} \tilde{\subset} J_{v} \cup E_{k} \cup F_{k} .
$$

To see this, we first observe that for $\mathcal{H}^{d-1}$-a.e. $x \in \Gamma=\bigcup_{i=1}^{\infty} J_{v_{i}}$ it holds that

$$
[v](x)=\left[u_{k}\right](x)+\sum_{i=k+1}^{\infty} c_{i}\left[v_{i}\right](x) .
$$


Moreover, we get that $c_{i} \leqq \varepsilon_{k} d_{i} 2^{-i-1}$ for all $i \geqq k+1$ by (4.13) and the fact that $\left(\varepsilon_{i}\right)_{i}$ is decreasing. Fix $x \in J_{u_{k}} \backslash\left(E_{k} \cup F_{k}\right)$. Then by (4.14) and (4.17) we get

$$
\begin{aligned}
|[v](x)| & \geqq\left|\left[u_{k}\right](x)\right|-\sum_{i=k+1}^{\infty} c_{i}\left|\left[v_{i}\right](x)\right| \geqq \varepsilon_{k}-\sum_{i=k+1}^{\infty} \frac{c_{i}}{d_{i}} \\
& \geqq \varepsilon_{k}\left(1-\sum_{i=k+1}^{\infty} 2^{-i-1}\right) \geqq \varepsilon_{k} / 2,
\end{aligned}
$$

where we have used that $\left|\left[u_{k}\right](x)\right| \geqq \varepsilon_{k}$ and $\left[v_{i}\right](x) \leqq 1 / d_{i}$, for $i \geqq k+1$. Thus, $[v](x) \neq 0$ and therefore $x \in J_{v}$. Consequently, we have shown that $\mathcal{H}^{d-1}$-a.e. $x \in J_{u_{k}} \backslash\left(E_{k} \cup F_{k}\right)$ lies in $J_{v}$. This shows (4.16).

We now conclude the proof as follows: by (4.12)(i) and (4.16), we get that

$$
\bigcup_{i=1}^{l} J_{v_{i}} \stackrel{=}{=} J_{u_{l}} \tilde{\subset} J_{v} \cup E_{l} \cup F_{l} \tilde{\subset} J_{v} \cup E_{k} \cup F_{k}
$$

for all $l \geqq k$, where we used that the sets $\left(E_{k}\right)_{k}$ and $\left(F_{k}\right)_{k}$ are decreasing. Taking the union with respect to $l$, we get that $\Gamma \tilde{\subset} J_{v} \cup E_{k} \cup F_{k}$ for all $k \in \mathbb{N}$. By (4.15) this implies $\mathcal{H}^{d-1}\left(\Gamma \backslash J_{v}\right) \leqq 2^{2-k}$. Since $k \in \mathbb{N}$ was arbitrary, we get $\Gamma \tilde{\subset} J_{v}$. This along with (4.11), shows that $J_{v} \stackrel{\sim}{=} \Gamma$, and concludes the proof.

We close this section with the proof of Lemma 4.4.

Proof of Lemma 4.4. Let $B=\left\{x \in V: \lim \sup _{n \rightarrow \infty}\left|u_{n}(x)-v_{n}(x)\right|<+\infty\right\}$. For $\theta \in(0,1)$, define $w_{n}^{\theta}=(1-\theta) u_{n}+\theta v_{n}$ and observe that $\left|w_{n}^{\theta}\right| \rightarrow \infty$ on $B$ for all $\theta$ since $\left|u_{n}\right| \rightarrow \infty$ on $V$. Let $D_{\theta}=\left\{x \in V \backslash B: \lim _{\sup _{n \rightarrow \infty}}\left|w_{n}^{\theta}(x)\right|<+\infty\right\}$. As $\left|u_{n}-v_{n}\right| \rightarrow \infty$ on $V \backslash B$ and thus $\left|w_{n}^{\theta_{1}}-w_{n}^{\theta_{2}}\right|=\left|\left(\theta_{1}-\theta_{2}\right)\left(v_{n}-u_{n}\right)\right| \rightarrow \infty$ on $V \backslash B$ for all $\theta_{1} \neq \theta_{2}$, we obtain $D_{\theta_{1}} \cap D_{\theta_{2}}=\emptyset$. This implies that $\mathcal{L}^{d}\left(D_{\theta}\right)>0$ for an at most countable number of different $\theta$. We note that for all $\theta$ with $\mathcal{L}^{d}\left(D_{\theta}\right)=0$ there holds $\left|w_{n}^{\theta}\right| \rightarrow \infty$ a.e. on $V$. This yields the claim.

\section{Functionals defined on Pairs of Function-Set}

This section is devoted to the proofs of the results announced in Section 2.1. Before proving the relaxation and existence results, we address the lower bound separately since this will be instrumental also for Section 6 .

\subsection{The Lower Bound}

In this subsection we prove a lower bound for functionals defined on pairs of function-set which will be needed for the proof of Theorem 2.2-Theorem 2.4. We will make use of the definition of $\operatorname{SSBD}_{\infty}^{p}(\Omega)$ in Section 3.4. In particular, we refer to the definition of $e(u)$ and of the jump set $J_{u}$ with its normal $v_{u}$, see (3.11)-(3.12), as well as to the notion of weak convergence in $\operatorname{GSBD}_{\infty}^{p}(\Omega)$, see (3.16). We recall also that for any $s \in[0,1]$ and any $E \in \mathfrak{M}(\Omega), E^{s}$ denotes the set of points with density $s$ for $E$, see [3, Definition 3.60]. 
Theorem 5.1. (Lower bound) Let $\Omega \subset \mathbb{R}^{d}$ be open and bounded, let $1<p<\infty$. Consider a sequence of Lipschitz sets $\left(E_{n}\right)_{n} \subset \Omega$ with $\sup _{n \in \mathbb{N}} \mathcal{H}^{d-1}\left(\partial E_{n}\right)<$ $+\infty$ and a sequence of functions $\left(u_{n}\right)_{n} \subset G S B D^{p}(\Omega)$ such that $\left.u_{n}\right|_{\Omega \backslash \overline{E_{n}}} \in$ $W^{1, p}\left(\Omega \backslash \overline{E_{n}} ; \mathbb{R}^{d}\right)$ and $u_{n}=0$ in $E_{n}$. Let $u \in G S B D_{\infty}^{p}(\Omega)$ and $E \in \mathfrak{M}(\Omega)$ such that $u_{n}$ converges weakly in $G S B D_{\infty}^{p}(\Omega)$ to $u$ and

$$
\chi_{E_{n}} \rightarrow \chi_{E} \text { in } L^{1}(\Omega)
$$

Then, for any norm $\varphi$ on $\mathbb{R}^{d}$ it holds that

$$
\begin{aligned}
& e\left(u_{n}\right) \chi \Omega \backslash\left(E_{n} \cup A_{u}^{\infty}\right)-e(u) \chi \Omega \backslash\left(E \cup A_{u}^{\infty}\right) \quad \text { weakly in } L^{p}\left(\Omega ; \mathbb{M}_{\text {sym }}^{d \times d}\right), \\
& \int_{J_{u} \cap E^{0}} 2 \varphi\left(v_{u}\right) \mathrm{d} \mathcal{H}^{d-1}+\int_{\Omega \cap \partial^{*} E} \varphi\left(v_{E}\right) \mathrm{d} \mathcal{H}^{d-1} \leqq \liminf _{n \rightarrow+\infty} \int_{\Omega \cap \partial E_{n}} \varphi\left(v_{E_{n}}\right) \mathrm{d} \mathcal{H}^{d-1},
\end{aligned}
$$

where $A_{u}^{\infty}=\{u=\infty\}$.

In the proof, we need the following two auxiliary results, (see [11, Proposition 4, Lemma 5]):

Proposition 5.2. Let $\Omega$ be an open subset of $\mathbb{R}^{d}$ and $\mu$ be a finite, positive set function defined on the family of open subsets of $\Omega$. Let $\lambda \in \mathcal{M}_{b}^{+}(\Omega)$, and $\left(g_{i}\right)_{i \in \mathbb{N}}$ be a family of positive Borel functions on $\Omega$. Assume that $\mu(U) \geqq \int_{U} g_{i} \mathrm{~d} \lambda$ for every $U$ and $i$, and that $\mu(U \cup V) \geqq \mu(U)+\mu(V)$ whenever $U, V \subset \subset \Omega$ and $\bar{U} \cap \bar{V}=\emptyset$ (superadditivity). Then $\mu(U) \geqq \int_{U}\left(\sup _{i \in \mathbb{N}} g_{i}\right) \mathrm{d} \lambda$ for every open $U \subset \Omega$.

Lemma 5.3. Let $\Gamma \subset E^{0}$ be a $(d-1)$-rectifiable subset, $\xi \in \mathbb{S}^{d-1}$ such that $\xi$ is not orthogonal to the normal $v_{\Gamma}$ to $\Gamma$ at any point of $\Gamma$. Then, for $\mathcal{H}^{d-1}$-a.e. $y \in \Pi \xi$, the set $E_{y}^{\xi}$ (see (3.1)) has density 0 in $t$ for every $t \in \Gamma_{y}^{\xi}$.

Proof of Theorem 5.1. Since $u_{n}$ converges weakly in $\operatorname{GSBD}_{\infty}^{p}(\Omega)$ to $u$, (3.16) implies

$$
\sup _{n \in \mathbb{N}}\left(\left\|e\left(u_{n}\right)\right\|_{L^{p}(\Omega)}^{p}+\mathcal{H}^{d-1}\left(J_{u_{n}}\right)+\mathcal{H}^{d-1}\left(\partial E_{n}\right)\right)=: M<+\infty .
$$

Consequently, Theorem 3.5 and the fact that $\bar{d}\left(u_{n}, u\right) \rightarrow 0$, see (3.13) and (3.16), imply that $A_{u}^{\infty}=\{u=\infty\}=\left\{x \in \Omega:\left|u_{n}(x)\right| \rightarrow \infty\right\}$ and

$$
\begin{gathered}
u_{n} \rightarrow u \quad \mathcal{L}^{d} \text {-a.e. in } \Omega \backslash A_{u}^{\infty}, \\
e\left(u_{n}\right) \rightarrow e(u) \quad \text { weakly in } L^{p}\left(\Omega \backslash A_{u}^{\infty} ; \mathbb{M}_{\text {sym }}^{d \times d}\right) .
\end{gathered}
$$

By (5.1), (5.4a), $u_{n}=0$ on $E_{n}$, and the definition of $A_{u}^{\infty}$, we have

$$
E \cap A_{u}^{\infty}=\varnothing \quad \text { and } \quad u=0 \mathcal{L}^{d} \text {-a.e. in } E .
$$

Then (5.4b) gives (5.2a). 
We now show (5.2b) which is the core of the proof. Let $\varphi^{*}$ be the dual norm of $\varphi$ and observe that (see, for example [10, Section 4.1.2])

$$
\varphi(v)=\max _{\xi \in \mathbb{S}^{d-1}} \frac{v \cdot \xi}{\varphi^{*}(\xi)}=\max _{\xi \in \mathbb{S}^{d-1}} \frac{|v \cdot \xi|}{\varphi^{*}(\xi)},
$$

where the second equality holds since $\varphi(v)=\varphi(-v)$.

As a preparatory step, we consider a set $B \subset \Omega$ with Lipschitz boundary and a function $v$ with $\left.v\right|_{\Omega \backslash \bar{B}} \in W^{1, p}\left(\Omega \backslash \bar{B} ; \mathbb{R}^{d}\right.$ ) and $v=0$ in $B$ (observe that $\left.v \in G S B D^{p}(\Omega)\right)$. Recall the notation in (3.1)-(3.2). Let $\varepsilon \in(0,1)$ and $U \subset \Omega$ be open. For each $\xi \in \mathbb{S}^{d-1}$ and $y \in \Pi^{\xi}$, we define

$$
F_{\varepsilon}^{\xi}\left(\widehat{v}_{y}^{\xi}, B_{y}^{\xi} ; U_{y}^{\xi}\right)=\varepsilon \int_{U_{y}^{\xi} \backslash B_{y}^{\xi}}\left|\left(\widehat{v}_{y}^{\xi}\right)^{\prime}\right|^{p} \mathrm{~d} t+\mathcal{H}^{0}\left(\partial B_{y}^{\xi} \cap U_{y}^{\xi}\right) \frac{1}{\varphi^{*}(\xi)} .
$$

By Fubini-Tonelli theorem, with the slicing properties (3.3), (3.4), (3.5), for a.e. $\xi \in \mathbb{S}^{d-1}$ it holds that

$$
\int_{\Pi \xi} F_{\varepsilon}^{\xi}\left(\widehat{v}_{y}^{\xi}, B_{y}^{\xi} ; U_{y}^{\xi}\right) \mathrm{d} \mathcal{H}^{d-1}(y)=\varepsilon \int_{U \backslash B}|e(v) \xi \cdot \xi|^{p} \mathrm{~d} x+\int_{U \cap \partial B} \frac{\left|v_{B} \cdot \xi\right|}{\varphi^{*}(\xi)} \mathrm{d} \mathcal{H}^{d-1} .
$$

Since $|e(v)| \geqq|e(v) \xi \cdot \xi|$, the previous estimate along with (5.6) implies

$$
\int_{\Pi \xi} F_{\varepsilon}^{\xi}\left(\widehat{v}_{y}^{\xi}, B_{y}^{\xi} ; U_{y}^{\xi}\right) \mathrm{d} \mathcal{H}^{d-1}(y) \leqq \varepsilon\|e(v)\|_{L^{p}(U \backslash B)}^{p}+\int_{U \cap \partial B} \varphi\left(v_{B}\right) \mathrm{d} \mathcal{H}^{d-1} .
$$
that,

By applying this estimate for the sequence of pairs $\left(u_{n}, E_{n}\right)$, we get, by (5.3)

$$
\begin{aligned}
\int_{\Pi \xi} F_{\varepsilon}^{\xi}\left(\left(\widehat{u}_{n}\right)_{y}^{\xi},\left(E_{n}\right)_{y}^{\xi} ; U_{y}^{\xi}\right) \mathrm{d} \mathcal{H}^{d-1}(y) & \leqq M \varepsilon+\int_{U \cap \partial E_{n}} \varphi\left(v_{E_{n}}\right) \mathrm{d} \mathcal{H}^{d-1} \\
& \leqq M\left(\|\varphi\|_{L^{\infty}\left(\mathbb{S}^{d-1}\right)}+\varepsilon\right)
\end{aligned}
$$

for all open $U \subset \Omega$. Since $\bar{d}\left(u_{n}, u\right) \rightarrow 0$, we have that $\bar{d}\left(\left(\widehat{u}_{n}\right)_{y}^{\xi}, \widehat{u}_{y}^{\xi}\right)=\int_{\Omega_{y}^{\xi}} d_{\overline{\mathbb{R}}^{d}}$ $\left(\left(\widehat{u}_{n}\right)_{y}^{\xi}, \widehat{u}_{y}^{\xi}\right) \mathrm{d} x \rightarrow 0$ for $\mathcal{H}^{d-1}$-a.e. $y \in \Pi^{\xi}$ and $\mathcal{H}^{d-1}$-a.e. $\xi \in \mathbb{S}^{d-1}$. (Notice that we have to restrict our choice to the $\xi$ satisfying $\left|u_{n} \cdot \xi\right| \rightarrow+\infty \mathcal{L}^{d}$-a.e. in $A_{u}^{\infty}$, which are a set of full measure in $\mathbb{S}^{d-1}$, cf. [19, Lemma 2.7].) In particular, this implies that

$$
\begin{aligned}
\left(\widehat{u}_{n}\right)_{y}^{\xi} \rightarrow \widehat{u}_{y}^{\xi} & \mathcal{L}^{1} \text {-a.e. in }\left(\Omega \backslash A_{u}^{\infty}\right)_{y}^{\xi}, \\
\left|\left(\widehat{u}_{n}\right)_{y}^{\xi}\right| \rightarrow+\infty & \mathcal{L}^{1} \text {-a.e. in }\left(A_{u}^{\infty}\right)_{y}^{\xi} .
\end{aligned}
$$

By using (5.8) and Fatou's lemma we obtain that

$$
\liminf _{n \rightarrow \infty} F_{\varepsilon}^{\xi}\left(\left(\widehat{u}_{n}\right)_{y}^{\xi},\left(E_{n}\right)_{y}^{\xi} ; U_{y}^{\xi}\right)<+\infty
$$

for $\mathcal{H}^{d-1}$-a.e. $y \in \Pi^{\xi}$ and any open $U \subset \Omega$. Then, we may find a subsequence $\left(u_{m}\right)_{m}=\left(u_{n_{m}}\right)_{m}$, depending on $\varepsilon$, $\xi$, and $y$, such that

$$
\lim _{m \rightarrow \infty} F_{\varepsilon}^{\xi}\left(\left(\widehat{u}_{m}\right)_{y}^{\xi},\left(E_{m}\right)_{y}^{\xi} ; U_{y}^{\xi}\right)=\liminf _{n \rightarrow \infty} F_{\varepsilon}^{\xi}\left(\left(\widehat{u}_{n}\right)_{y}^{\xi},\left(E_{n}\right)_{y}^{\xi} ; U_{y}^{\xi}\right)
$$


for any open $U \subset \Omega$. At this stage, up to passing to a further subsequence, we have

$$
\mathcal{H}^{0}\left(\partial\left(E_{m}\right)_{y}^{\xi}\right)=N_{y}^{\xi} \in \mathbb{N}
$$

independently of $m$, so that the points in $\partial\left(E_{m}\right)_{y}^{\xi}$ converge, as $m \rightarrow \infty$, to $M_{y}^{\xi} \leqq N_{y}^{\xi}$ points

$$
t_{1}, \ldots, t_{M_{y}^{\xi}}^{\xi}
$$

which are either in $\partial E_{y}^{\xi}$ or in a finite set $S_{y}^{\xi}:=\left\{t_{1}, \ldots, t_{M_{y}^{\xi}}\right\} \backslash \partial E_{y}^{\xi} \subset\left(E_{y}^{\xi}\right)^{0} \cup\left(E_{y}^{\xi}\right)^{1}$, where $(\cdot)^{0}$ and $(\cdot)^{1}$ denote the sets with one-dimensional density 0 or 1 , respectively. Notice that $E_{y}^{\xi}$ is thus the union of $M_{y}^{\xi} / 2-\# S_{y}^{\xi}$ intervals (up to a finite set of points) on which there holds $\widehat{u}_{y}^{\xi}=0$, see (5.5) and (5.9a). In view of (5.7) and (5.10), $\left(\left(\widehat{u}_{m}\right)_{y}^{\xi}\right)^{\prime}$ are equibounded (with respect to $\left.m\right)$ in $L_{\text {loc }}^{p}\left(t_{j}, t_{j+1}\right)$, for any interval

$$
\left(t_{j}, t_{j+1}\right) \subset \Omega_{y}^{\xi} \backslash\left(E_{y}^{\xi} \cup S_{y}^{\xi}\right) .
$$

Then, as in the proof of [19, Theorem 1.1], we have two alternative possibilities on $\left(t_{j}, t_{j+1}\right)$ : either $\left(\widehat{u}_{m}\right)_{y}^{\xi}$ converge locally uniformly in $\left(t_{j}, t_{j+1}\right)$ to $\widehat{u}_{y}^{\xi}$, or $\left|\left(\widehat{u}_{m}\right)_{y}^{\xi}\right| \rightarrow$ $+\infty \mathcal{L}^{1}$-a.e. in $\left(t_{j}, t_{j+1}\right)$. Recalling that $J_{\widehat{u}_{y}^{\xi}}=\partial\left(A_{u}^{\infty}\right)_{y}^{\xi} \cup\left(\left(J_{u}^{\xi}\right)_{y}^{\xi} \backslash\left(A_{u}^{\infty}\right)_{y}^{\xi}\right)$, see (3.4a) and (3.11), we find that

$$
J_{\xi, y}:=J_{\widehat{u}_{y}^{\xi}} \cap\left(E_{y}^{\xi}\right)^{0} \subset S_{y}^{\xi} \cap\left(E_{y}^{\xi}\right)^{0} .
$$

We notice that any point in $S_{y}^{\xi}$ is the limit of two distinct sequences of points $\left(p_{m}^{1}\right)_{m}$, $\left(p_{m}^{2}\right)_{m}$ with $p_{m}^{1}, p_{m}^{2} \in \partial\left(E_{m}\right)_{y}^{\xi}$. Thus, in view of (5.7) and (5.11), for any open $U \subset \Omega$ we derive

$$
\begin{aligned}
& \varepsilon \int_{U_{y}^{\xi} \backslash\left(E \cup A_{u}^{\infty}\right)_{y}^{\xi}}\left|\left(\widehat{u}_{y}^{\xi}\right)^{\prime}\right|^{p} \mathrm{~d} t+\mathcal{H}^{0}\left(U_{y}^{\xi} \cap \partial E_{y}^{\xi}\right) \frac{1}{\varphi^{*}(\xi)}+\mathcal{H}^{0}\left(U_{y}^{\xi} \cap J_{\xi, y}\right) \frac{2}{\varphi^{*}(\xi)} \\
& \quad \leqq \liminf _{m \rightarrow \infty} F_{\varepsilon}^{\xi}\left(\left(\widehat{u}_{m}\right)_{y}^{\xi},\left(E_{m}\right)_{y}^{\xi} ; U_{y}^{\xi}\right)=\liminf _{n \rightarrow \infty} F_{\varepsilon}^{\xi}\left(\left(\widehat{u}_{n}\right)_{y}^{\xi},\left(E_{n}\right)_{y}^{\xi} ; U_{y}^{\xi}\right) .
\end{aligned}
$$

We apply Lemma 5.3 to the rectifiable set $J_{u} \cap E^{0} \cap\left\{\xi \cdot v_{u} \neq 0\right\}$ and get that, for $\mathcal{H}^{d-1}$-a.e. $y \in \Pi^{\xi}$,

$$
y+t \xi \in J_{u} \cap E^{0} \cap\left\{\xi \cdot v_{u} \neq 0\right\} \quad \Rightarrow \quad t \in\left(E_{y}^{\xi}\right)^{0} .
$$

This along with (5.12)-(5.13), the slicing properties (3.3)-(3.5) (which also hold for $G S B D_{\infty}^{p}(\Omega)$ functions), and Fatou's lemma yields that for all $\xi \in \mathbb{S}^{d-1} \backslash N_{0}$, for some $N_{0}$ with $\mathcal{H}^{d-1}\left(N_{0}\right)=0$, it holds that

$$
\begin{aligned}
& \varepsilon \int_{U \backslash\left(E \cup A_{u}^{\infty}\right)}|e(u) \xi \cdot \xi|^{p} \mathrm{~d} x+\int_{U \cap \partial^{*} E} \frac{\left|v_{E} \cdot \xi\right|}{\varphi^{*}(\xi)} \mathrm{d} \mathcal{H}^{d-1} \\
& \quad+\int_{J_{u} \cap E^{0} \cap U} \frac{2\left|v_{u} \cdot \xi\right|}{\varphi^{*}(\xi)} \mathrm{d} \mathcal{H}^{d-1} \\
& \leqq \int_{\Pi^{\xi}} \liminf _{n \rightarrow \infty} F_{\varepsilon}^{\xi}\left(\left(\widehat{u}_{n}\right)_{y}^{\xi},\left(E_{n}\right)_{y}^{\xi} ; U_{y}^{\xi}\right) \mathrm{d} \mathcal{H}^{d-1}
\end{aligned}
$$




$$
\leqq \liminf _{n \rightarrow \infty} \int_{\Pi^{\xi}} F_{\varepsilon}^{\xi}\left(\left(\widehat{u}_{n}\right)_{y}^{\xi},\left(E_{n}\right)_{y}^{\xi} ; U_{y}^{\xi}\right) \mathrm{d} \mathcal{H}^{d-1} .
$$

Introducing the set function $\mu$ defined on the open subsets of $\Omega$ by

$$
\mu(U):=\liminf _{n \rightarrow+\infty} \int_{U \cap \partial E_{n}} \varphi\left(v_{E_{n}}\right) \mathrm{d} \mathcal{H}^{d-1},
$$

and letting $\varepsilon \rightarrow 0$ we find by (5.8) for all $\xi \in \mathbb{S}^{d-1} \backslash N_{0}$ that

$$
\int_{U \cap \partial^{*} E} \frac{\left|v_{E} \cdot \xi\right|}{\varphi^{*}(\xi)} \mathrm{d} \mathcal{H}^{d-1}+\int_{J_{u} \cap E^{0} \cap U} \frac{2\left|v_{u} \cdot \xi\right|}{\varphi^{*}(\xi)} \mathrm{d} \mathcal{H}^{d-1} \leqq \mu(U) .
$$

The set function $\mu$ is clearly superadditive. Let $\lambda=\mathcal{H}^{d-1}\left\llcorner\left(J_{u} \cap E^{0}\right)+\mathcal{H}^{d-1}\left\llcorner\partial^{*} E\right.\right.$ and define

$$
g_{i}= \begin{cases}\frac{2\left|v_{u} \cdot \xi_{i}\right|}{\varphi^{*}\left(\xi_{i}\right)} & \text { on } J_{u} \cap E^{0} \\ \frac{\left|v_{E} \cdot \xi_{i}\right|}{\varphi^{*}\left(\xi_{i}\right)} & \text { on } \partial^{*} E\end{cases}
$$

where $\left(\xi_{i}\right)_{i} \subset \mathbb{S}^{d-1} \backslash N_{0}$ is a dense sequence in $\mathbb{S}^{d-1}$. By (5.15) we have $\mu(U) \geqq$ $\int_{U} g_{i} \mathrm{~d} \lambda$ for all $i \in \mathbb{N}$ and all open $U \subset \Omega$. Then, Proposition 5.2 yields $\mu(\Omega) \geqq$ $\int_{\Omega} \sup _{i} g_{i} \mathrm{~d} \lambda$. In view of (5.6) and (5.14), this implies (5.2b) and concludes the proof.

\subsection{Relaxation for Functionals defined on Pairs of Function-Set}

In this subsection we give the proof of Proposition 2.1 and Theorem 2.2. We also provide corresponding generalizations to the space $G S B D_{\infty}^{p}$, see Proposition 5.5 and Theorem 5.8. For the upper bound, we recall the following result proved in [11, Proposition 9, Remark 14]:

Proposition 5.4. Let $u \in L^{1}\left(\Omega ; \mathbb{R}^{d}\right)$ and $E \in \mathfrak{M}(\Omega)$ such that $\mathcal{H}^{d-1}\left(\partial^{*} E\right)<$ $+\infty$ and $u \chi_{E^{0}} \in G S B V^{p}\left(\Omega ; \mathbb{R}^{d}\right)$. Then, there exists a sequence $\left(u_{n}\right)_{n} \subset W^{1, p}$ $\left(\Omega ; \mathbb{R}^{d}\right)$ and $\left(E_{n}\right)_{n} \subset \mathfrak{M}(\Omega)$ with $E_{n}$ of class $C^{\infty}$ such that $u_{n} \rightarrow$ u in $L^{1}\left(\Omega ; \mathbb{R}^{d}\right)$, $\chi_{E_{n}} \rightarrow \chi_{E}$ in $L^{1}(\Omega)$, and

$$
\begin{aligned}
& \nabla u_{n} \chi_{\Omega \backslash E_{n}} \rightarrow \nabla u \chi_{\Omega \backslash E} \quad \text { in } L^{p}\left(\Omega ; \mathbb{M}^{d \times d}\right) \\
& \quad \limsup _{n \rightarrow \infty} \int_{\partial E_{n} \cap \Omega} \varphi\left(v_{E_{n}}\right) \mathrm{d} \mathcal{H}^{d-1} \leqq \int_{J_{u} \cap E^{0}} 2 \varphi\left(v_{u}\right) \mathrm{d} \mathcal{H}^{d-1}+\int_{\partial^{*} E \cap \Omega} \varphi\left(v_{E}\right) \mathrm{d} \mathcal{H}^{d-1}
\end{aligned}
$$

Moreover, if $\mathcal{L}^{d}(E)>0$, one can guarantee in addition the condition $\mathcal{L}^{d}\left(E_{n}\right)=$ $\mathcal{L}^{d}(E)$ for $n \in \mathbb{N}$.

Proof of Proposition 2.1. We first prove the lower inequality, and then the upper inequality. The lower inequality relies on Theorem 5.1 and the upper inequality on a density argument along with Proposition 5.4.

The lower inequality. Suppose that $u_{n} \rightarrow u$ in $L^{0}\left(\Omega ; \mathbb{R}^{d}\right)$ and $\chi_{E_{n}} \rightarrow \chi_{E}$ in $L^{1}(\Omega)$. Without restriction, we can assume that $\sup _{n} F\left(u_{n}, E_{n}\right)<+\infty$. In view of (2.2) and $\min _{\mathbb{S}^{d-1}} \varphi>0$, this implies $\mathcal{H}^{d-1}\left(\partial E_{n}\right)<+\infty$. Moreover, by (2.1) the 
functions $v_{n}:=u_{n} \chi \Omega \backslash E_{n}$ lie in $\operatorname{GSBD}^{p}(\Omega)$ with $J_{v_{n}} \subset \partial E_{n} \cap \Omega$ and satisfy $\sup _{n}\left\|e\left(v_{n}\right)\right\|_{L^{p}(\Omega)}<+\infty$. This along with the fact that $u_{n} \rightarrow u$ in measure shows that $v_{n}$ converges weakly in $G S B D_{\infty}^{p}(\Omega)$ to $u \chi_{E^{0}}$, see (3.16), where we point out that $A_{u}^{\infty}=\{u=\infty\}=\emptyset$. In particular, $u \chi_{E^{0}} \in G S B D_{\infty}^{p}(\Omega)$ and, since $A_{u}^{\infty}=\emptyset$, even $u \chi_{E^{0}} \in G S B D^{p}(\Omega)$, cf. (3.10). As also (5.1) holds, we can apply Theorem 5.1. The lower inequality now follows from (5.2) and the fact that $f$ is convex.

The upper inequality. We first observe the following: given $u \in L^{0}\left(\Omega ; \mathbb{R}^{d}\right)$ and $E \in \mathfrak{M}(\Omega)$ with $\mathcal{H}^{d-1}\left(\partial^{*} E\right)<\infty$ and $u \chi_{E^{0}} \in G S B D^{p}(\Omega)$, we find an approximating sequence $\left(v_{n}\right)_{n} \subset L^{1}\left(\Omega ; \mathbb{R}^{d}\right)$ with $v_{n} \chi_{E^{0}} \in G S B V^{p}\left(\Omega ; \mathbb{R}^{d}\right)$ such that

$$
\begin{aligned}
& \text { (i) } v_{n} \rightarrow u \chi_{E^{0}} \quad \text { in } L^{0}\left(\Omega ; \mathbb{R}^{d}\right) \text {, } \\
& \text { (ii) } e\left(v_{n}\right) \chi_{\Omega \backslash E} \rightarrow e(u) \chi_{\Omega \backslash E} \text { in } L^{p}\left(\Omega ; \mathbb{M}_{\mathrm{sym}}^{d \times d}\right) \text {, } \\
& \text { (iii) } \mathcal{H}^{d-1}\left(\left(J_{v_{n}} \triangle J_{u}\right) \cap E^{0}\right) \rightarrow 0 .
\end{aligned}
$$

This can be seen by approximating first $u \chi_{E^{0}}$ by a sequence $\left(\tilde{u}_{n}\right)_{n}$ by means of Theorem 3.4, and by setting $v_{n}:=\widetilde{u}_{n} \chi_{E^{0}}$ for every $n$. It is then immediate to verify that the conditions in (3.6) for $\left(\tilde{u}_{n}\right)_{n}$ imply the three conditions above.

By this approximation, (2.1), and a diagonal argument, it thus suffices to construct a recovery sequence for $u \in L^{1}\left(\Omega ; \mathbb{R}^{d}\right)$ with $u \chi_{E^{0}} \in G S B V^{p}\left(\Omega ; \mathbb{R}^{d}\right)$. To this end, we apply Proposition 5.4 to obtain $\left(u_{n}, E_{n}\right)_{n}$ and we consider the sequence $u_{n} \chi_{\Omega \backslash E_{n}}$. We further observe that, if $\mathcal{L}^{d}(E)>0$, this recovery sequences $\left(u_{n}, E_{n}\right)_{n}$ can be constructed ensuring $\mathcal{L}^{d}\left(E_{n}\right)=\mathcal{L}^{d}(E)$ for $n \in \mathbb{N}$.

We briefly discuss that by a small adaption we get a relaxation result for $F$ with respect to the topology induced by $\bar{d}$ on $L^{0}\left(\Omega ; \overline{\mathbb{R}}^{d}\right)$. We introduce $\bar{F}_{\infty}: L^{0}\left(\Omega ; \overline{\mathbb{R}}^{d}\right) \times$ $\mathfrak{M}(\Omega) \rightarrow \mathbb{R} \cup\{+\infty\}$ by

$$
\bar{F}_{\infty}(u, E)=\inf \left\{\liminf _{n \rightarrow \infty} F\left(u_{n}, E_{n}\right): \bar{d}\left(u_{n}, u\right) \rightarrow 0 \text { and } \chi_{E_{n}} \rightarrow \chi_{E} \text { in } L^{1}(\Omega)\right\} .
$$

Proposition 5.5. (Characterization of the lower semicontinuous envelope $\bar{F}_{\infty}$ ) Under the assumptions of Proposition 2.1, it holds that

$$
\bar{F}_{\infty}(u, E)=\left\{\begin{array}{cl}
\int_{\Omega \backslash E} f(e(u)) \mathrm{d} x+\int_{\Omega \cap \partial^{*} E} \varphi\left(v_{E}\right) \mathrm{d} \mathcal{H}^{d-1}+\int_{J_{u} \cap(\Omega \backslash E)^{1}} 2 \varphi\left(v_{u}\right) \mathrm{d} \mathcal{H}^{d-1} \\
\text { if } u=u \chi_{E^{0}} \in G S B D_{\infty}^{p}(\Omega) \text { and } \mathcal{H}^{d-1}\left(\partial^{*} E\right)<+\infty, \\
\text { otherwise. }
\end{array}\right.
$$

Moreover, if $\mathcal{L}^{d}(E)>0$, then for any $(u, E) \in L^{0}\left(\Omega ; \overline{\mathbb{R}}^{d}\right) \times \mathfrak{M}(\Omega)$ there exists a recovery sequence $\left(u_{n}, E_{n}\right)_{n} \subset L^{0}\left(\Omega ; \mathbb{R}^{d}\right) \times \mathfrak{M}(\Omega)$ such that $\mathcal{L}^{d}\left(E_{n}\right)=\mathcal{L}^{d}(E)$ for all $n \in \mathbb{N}$.

Proof. It is easy to check that the lower inequality still works for $u=u \chi_{E^{0}} \in$ $G S B D_{\infty}^{p}(\Omega)$ by Theorem 5.1, where we use (2.1), $f(0)=0$, and the fact that $e(u)=0$ on $\{u=\infty\}$, see (3.12). Moreover, we are able to extend the upper inequality to any $u \in L^{0}\left(\Omega ; \overline{\mathbb{R}}^{d}\right)$ such that $u=u \chi_{E^{0}} \in G S B D_{\infty}^{p}(\Omega)$. In fact, it is enough to notice that for any $u=u \chi_{E^{0}} \in G S B D_{\infty}^{p}(\Omega)$ and any sequence 
$\left(t_{n}\right)_{n} \subset \mathbb{R}^{d}$ with $\left|t_{n}\right| \rightarrow \infty$ such that for the functions $\tilde{u}_{t_{n}} \in G S B D^{p}(\Omega)$ defined in (3.10) property (3.12) holds, we obtain $\tilde{u}_{t_{n}}=\tilde{u}_{t_{n}} \chi_{E^{0}}, \bar{d}\left(\tilde{u}_{t_{n}}, u\right) \rightarrow 0$ as $n \rightarrow \infty$, and

$\int_{\Omega \backslash E} f(e(u)) \mathrm{d} x+\int_{\Omega \cap \partial^{*} E} \varphi\left(v_{E}\right) \mathrm{d} \mathcal{H}^{d-1}+\int_{J_{u} \cap(\Omega \backslash E)^{1}} 2 \varphi\left(v_{u}\right) \mathrm{d} \mathcal{H}^{d-1}=\bar{F}\left(\tilde{u}_{t_{n}}, E\right)$

for all $n \in \mathbb{N}$, with $J_{u}$ defined by (3.11). Then, the upper inequality follows from the upper inequality in Proposition 2.1 and a diagonal argument.

As a consequence, we obtain the following lower semicontinuity result in $G S B D_{\infty}^{p}$ :

Corollary 5.6. (Lower semicontinuity in $G S B D_{\infty}^{p}$ ) Let us suppose that a sequence $\left(u_{n}\right)_{n} \subset G S B D_{\infty}^{p}(\Omega)$ converges weakly in $G S B D_{\infty}^{p}(\Omega)$ to $u \in G S B D_{\infty}^{p}(\Omega)$, see (3.16). Then for each norm $\phi$ on $\mathbb{R}^{d}$ it holds that

$$
\int_{J_{u}} \phi\left(v_{u}\right) \mathrm{d} \mathcal{H}^{d-1} \leqq \liminf _{n \rightarrow \infty} \int_{J_{u_{n}}} \phi\left(v_{u_{n}}\right) \mathrm{d} \mathcal{H}^{d-1} .
$$

Proof. Let $\varepsilon>0$ and $f(\zeta)=\varepsilon\left|\zeta^{T}+\zeta\right|^{p}$ for $\zeta \in \mathbb{M}^{d \times d}$. The upper inequality in Proposition 5.5 (for $u_{n}$ and $E=\emptyset$ ) shows that for each $u_{n} \in G S B D_{\infty}^{p}(\Omega)$, we can find a Lipschitz set $E_{n}$ with $\mathcal{L}^{d}\left(E_{n}\right) \leqq \frac{1}{n}$ and $v_{n} \in L^{0}\left(\Omega ; \mathbb{R}^{d}\right)$ with $\left.v_{n}\right|_{\Omega \backslash \bar{E}_{n}} \in W^{1, p}\left(\Omega \backslash \bar{E}_{n} ; \mathbb{R}^{d}\right),\left.v_{n}\right|_{E_{n}}=0$, and $\bar{d}\left(v_{n}, u_{n}\right) \leqq \frac{1}{n}$ (see (3.13)) such that

$$
\begin{aligned}
& \int_{\Omega \backslash E_{n}} \varepsilon\left|e\left(v_{n}\right)\right|^{p} \mathrm{~d} x+\int_{\Omega \cap \partial E_{n}} \phi\left(v_{E_{n}}\right) \mathrm{d} \mathcal{H}^{d-1} \leqq \int_{\Omega} \varepsilon\left|e\left(u_{n}\right)\right|^{p} \mathrm{~d} x \\
& \quad+\int_{J_{u_{n}}} 2 \phi\left(v_{u_{n}}\right) \mathrm{d} \mathcal{H}^{d-1}+\frac{1}{n} .
\end{aligned}
$$

Observe that $\bar{d}\left(v_{n}, u\right) \rightarrow 0$ as $n \rightarrow \infty$, and thus $v_{n}$ converges weakly to $u$ in $G S B D_{\infty}^{p}(\Omega)$. By applying Theorem 5.1 on $\left(v_{n}, E_{n}\right)$ and using $E=\emptyset$ we get

$$
\int_{J_{u}} 2 \phi\left(v_{u}\right) \mathrm{d} \mathcal{H}^{d-1} \leqq \liminf _{n \rightarrow \infty} \int_{\Omega \cap \partial E_{n}} \phi\left(v_{E_{n}}\right) \mathrm{d} \mathcal{H}^{d-1} .
$$

This, along with (5.16), $\sup _{n \in \mathbb{N}}\left\|e\left(u_{n}\right)\right\|_{L^{p}(\Omega)}<+\infty$, and the arbitrariness of $\varepsilon$ yields the result.

We now address the relaxation of $F_{\text {Dir }}$, see (2.5), that is, a version of $F$ with boundary data.

We take advantage of the following approximation result which is obtained by following the lines of [17, Theorem 5.5], where an analogous approximation is proved for Griffith functionals with Dirichlet boundary conditions. The new feature with respect to [17, Theorem 5.5] is that, besides the construction of approximating functions with the correct boundary data, also approximating sets are constructed. For convenience of the reader, we give a sketch of the proof in Appendix A highlighting the adaptations needed with respect to [17, Theorem 5.5]. In the following, we denote by $\bar{F}_{\text {Dir }}^{\prime}$ the functional on the right hand side of (2.7): 
Lemma 5.7. Suppose that $\partial_{D} \Omega \subset \partial \Omega$ satisfies (2.4). Consider $(v, H) \in L^{0}\left(\Omega ; \mathbb{R}^{d}\right)$ $\times \mathfrak{M}(\Omega)$ such that $\bar{F}_{\text {Dir }}^{\prime}(v, H)<+\infty$. Then there exist $\left(v_{n}, H_{n}\right) \in\left(L^{p}\left(\Omega ; \mathbb{R}^{d}\right) \cap\right.$ $\left.S B V^{p}\left(\Omega ; \mathbb{R}^{d}\right)\right) \times \mathfrak{M}(\Omega)$ such that $J_{v_{n}}$ is closed in $\Omega$ and included in a finite union of closed connected pieces of $C^{1}$ hypersurfaces, $v_{n} \in W^{1, p}\left(\Omega \backslash J_{v_{n}} ; \mathbb{R}^{d}\right), v_{n}=u_{0}$ in a neighborhood $V_{n} \subset \Omega$ of $\partial_{D} \Omega, H_{n}$ is a set of finite perimeter, and

(i) $v_{n} \rightarrow v$ in $L^{0}\left(\Omega ; \mathbb{R}^{d}\right)$,

(ii) $\chi_{H_{n}} \rightarrow \chi_{H}$ in $L^{1}(\Omega)$,

(iii) $\lim \sup _{n \rightarrow \infty} \bar{F}_{\text {Dir }}^{\prime}\left(v_{n}, H_{n}\right) \leqq \bar{F}_{\text {Dir }}^{\prime}(v, H)$.

Proof of Theorem 2.2. First, we denote by $\Omega^{\prime}$ a bounded open set with $\Omega \subset \Omega^{\prime}$ and $\Omega^{\prime} \cap \partial \Omega=\partial_{D} \Omega$. By $F^{\prime}$ and $\bar{F}^{\prime}$ we denote the analogs of the functionals $F$ and $\bar{F}$, respectively, defined on $L^{0}\left(\Omega^{\prime} ; \mathbb{R}^{d}\right) \times \mathfrak{M}\left(\Omega^{\prime}\right)$. Given $u \in L^{0}\left(\Omega ; \mathbb{R}^{d}\right)$, we define the extension $u^{\prime} \in L^{0}\left(\Omega^{\prime} ; \mathbb{R}^{d}\right)$ by setting $u^{\prime}=u_{0}$ on $\Omega^{\prime} \backslash \Omega$ for fixed boundary values $u_{0} \in W^{1, p}\left(\mathbb{R}^{d} ; \mathbb{R}^{d}\right)$. Then, we observe

$$
\begin{aligned}
F^{\prime}\left(u^{\prime}, E\right)= & F_{\text {Dir }}(u, E)+\int_{\Omega^{\prime} \backslash \Omega} f\left(e\left(u_{0}\right)\right) \mathrm{d} x, \quad \bar{F}^{\prime}\left(u^{\prime}, E\right)=\bar{F}_{\operatorname{Dir}}(u, E) \\
& +\int_{\Omega^{\prime} \backslash \Omega} f\left(e\left(u_{0}\right)\right) \mathrm{d} x .
\end{aligned}
$$

Therefore, the lower inequality follows from Proposition 2.1 applied for $F^{\prime}, \bar{F}^{\prime}$ instead of $F, \bar{F}$.

We now address the upper inequality. In view of Lemma 5.7 and by a diagonal argument, it is enough to prove the result in the case where, besides the assumptions in the statement, also $u \in L^{p}\left(\Omega ; \mathbb{R}^{d}\right) \cap S B V^{p}\left(\Omega ; \mathbb{R}^{d}\right)$ and $u=u_{0}$ in a neighborhood $U \subset \Omega$ of $\partial_{D} \Omega$.

By $\left(u_{n}, E_{n}\right)_{n}$ we denote a recovery sequence for $(u, E)$ given by Proposition 5.4. In general, the functions $\left(u_{n}\right)_{n}$ do not satisfy the boundary conditions required in (2.5). Let $\delta>0$ and let $V \subset \subset U$ be a smaller neighborhood of $\partial_{D} \Omega$. In view of (2.1)-(2.2), by a standard diagonal argument in the theory of $\Gamma$-convergence, it suffices to find a sequence $\left(v_{n}\right)_{n} \subset L^{1}\left(\Omega ; \mathbb{R}^{d}\right)$ with $\left.v_{n}\right|_{\Omega \backslash \bar{E}_{n}} \in W^{1, p}\left(\Omega \backslash \bar{E}_{n} ; \mathbb{R}^{d}\right)$, $v_{n}=0$ on $E_{n}$, and $v_{n}=u_{0}$ on $V \backslash \bar{E}_{n}$ such that

$\lim \sup _{n \rightarrow \infty}\left\|v_{n}-u \chi_{E^{0}}\right\|_{L^{1}(\Omega)} \leqq \delta, \quad \lim \sup _{n \rightarrow \infty}\left\|e\left(v_{n}\right)-e\left(u_{n}\right) \chi_{\Omega \backslash E_{n}}\right\|_{L^{p}(\Omega)} \leqq \delta$.

To this end, choose $\psi \in C^{\infty}(\Omega)$ with $\psi=1$ in $\Omega \backslash U$ and $\psi=0$ on $V$. The sequence $\left(u_{n}\right)_{n}$ converges to $u$ only in $L^{1}\left(\Omega ; \mathbb{R}^{d}\right)$. Therefore, we introduce truncations to obtain $L^{p}$-convergence: for $M>0$, we define $u^{M}$ by $u_{i}^{M}=\left(-M \vee u_{i}\right) \wedge M$, where $u_{i}$ denotes the $i$-th component, $i=1, \ldots, d$. In a similar fashion, we define $u_{n}^{M}$. By Proposition 5.4 we then get $\chi_{E_{n}} \rightarrow \chi_{E}$ in $L^{1}(\Omega)$ and

$u_{n}^{M} \rightarrow u^{M} \quad$ in $\quad L^{p}\left(\Omega ; \mathbb{R}^{d}\right), \quad \nabla u_{n}^{M} \chi_{\Omega \backslash E_{n}} \rightarrow \nabla u^{M} \chi_{E^{0}} \quad$ in $\quad L^{p}\left(\Omega ; \mathbb{M}^{d \times d}\right)$. 
We define $v_{n}:=\left(\psi u_{n}^{M}+(1-\psi) u_{0}\right) \chi_{\Omega \backslash E_{n}}$. Clearly, $v_{n}=u_{0}$ on $V \backslash \bar{E}_{n}$. By $\nabla v_{n}=\psi \nabla u_{n}^{M}+(1-\psi) \nabla u_{0}+\nabla \psi \otimes\left(u_{n}^{M}-u_{0}\right)$ on $\Omega \backslash E_{n}, u=u_{0}$ on $U$, and Proposition 5.4 we find

$$
\begin{aligned}
\lim \sup _{n \rightarrow \infty}\left\|v_{n}-u\right\|_{L^{1}(\Omega)} \leqq & \left\|u-u^{M}\right\|_{L^{1}(\Omega)}, \\
\lim \sup _{n \rightarrow \infty}\left\|e\left(v_{n}\right)-e\left(u_{n}\right) \chi_{\Omega \backslash E_{n}}\right\|_{L^{p}(\Omega)} \leqq & \left\|\nabla u-\nabla u^{M}\right\|_{L^{p}\left(E^{0}\right)} \\
& +\|\nabla \psi\|_{\infty}\left\|u-u^{M}\right\|_{L^{p}(\Omega)} .
\end{aligned}
$$

For $M$ sufficiently large, we obtain (5.18) since $u=u \chi_{E^{0}}$. This concludes the proof.

As done for the passage from Proposition 2.1 to Proposition 5.5, we may obtain the following characterization of the lower semicontinuous envelope of $F_{\text {Dir }}$ with respect to the convergence induced by $\bar{d}$ on $L^{0}\left(\Omega ; \overline{\mathbb{R}}^{d}\right)$.

Theorem 5.8. (Characterization of the lower semicontinuous envelope $\bar{F}_{\mathrm{Dir}, \infty}$ ) Under the assumptions of Theorem 2.2, the lower semicontinous envelope

$$
\bar{F}_{\operatorname{Dir}, \infty}(u, E)=\left\{\liminf _{n \rightarrow \infty} F_{\operatorname{Dir}}\left(u_{n}, E_{n}\right): \bar{d}\left(u_{n}, u\right) \rightarrow 0 \text { and } \chi_{E_{n}} \rightarrow \chi_{E} \text { in } L^{1}(\Omega)\right\}
$$

for $u \in L^{0}\left(\Omega ; \overline{\mathbb{R}}^{d}\right)$ and $E \in \mathfrak{M}(\Omega)$ is given by

$$
\bar{F}_{\operatorname{Dir}, \infty}(u, E)=\bar{F}_{\infty}(u, E)+\int_{\partial_{D} \Omega \cap \partial^{*} E} \varphi\left(v_{E}\right) \mathrm{d} \mathcal{H}^{d-1}+\int_{\left\{\operatorname{tr}(u) \neq \operatorname{tr}\left(u_{0}\right)\right\} \cap\left(\partial_{D} \Omega \backslash \partial^{*} E\right)} 2 \varphi\left(v_{\Omega}\right) \mathrm{d} \mathcal{H}^{d-1} .
$$

Moreover, if $\mathcal{L}^{d}(E)>0$, then for any $(u, E) \in L^{0}\left(\Omega ; \overline{\mathbb{R}}^{d}\right) \times \mathfrak{M}(\Omega)$ there exists a recovery sequence $\left(u_{n}, E_{n}\right)_{n} \subset L^{0}\left(\Omega ; \mathbb{R}^{d}\right) \times \mathfrak{M}(\Omega)$ such that $\mathcal{L}^{d}\left(E_{n}\right)=\mathcal{L}^{d}(E)$ for all $n \in \mathbb{N}$.

Notice that in (5.19) we wrote $\operatorname{tr}(u)$ also for $u \in G S B D_{\infty}^{p}(\Omega)$, with a slight abuse of notation: $\operatorname{tr}(u)$ should be intended as $\operatorname{tr}\left(\tilde{u}_{t}\right)$, cf. (3.10), for any $t \in \mathbb{R}^{d}$ such that $\mathcal{H}^{d-1}\left(\left\{u_{0}=t\right\} \cap \partial_{D} \Omega\right)=0$.

\subsection{Compactness and Existence Results for the Relaxed Functional}

We start with the following general compactness result:

Theorem 5.9. (Compactness) For every $\left(u_{n}, E_{n}\right)_{n}$ with $\sup _{n} F\left(u_{n}, E_{n}\right)<+\infty$, there exist a subsequence (not relabeled), $u \in G S B D_{\infty}^{p}(\Omega)$, and $E \in \mathfrak{M}(\Omega)$ with $\mathcal{H}^{d-1}\left(\partial^{*} E\right)<+\infty$ such that $u_{n}$ converges weakly in $\operatorname{GSBD}_{\infty}^{p}(\Omega)$ to $u$ and $\chi_{E_{n}} \rightarrow \chi_{E}$ in $L^{1}(\Omega)$.

Proof. Let $\left(u_{n}, E_{n}\right)_{n}$ with $\sup _{n} F\left(u_{n}, E_{n}\right)<+\infty$. As by the assumptions on $\varphi$ there holds $\sup _{n \in \mathbb{N}} \mathcal{H}^{d-1}\left(\partial E_{n}\right)<+\infty$, a compactness result for sets of finite perimeter (see [3, Remark 4.20]) implies that there exists $E \subset \Omega$ with $\mathcal{H}^{d-1}\left(\partial^{*} E\right)<+\infty$ such that $\chi_{E_{n}} \rightarrow \chi_{E}$ in $L^{1}(\Omega)$, up to a subsequence (not relabeled). 
Since the functions $u_{n}=u_{n} \chi \Omega \backslash E_{n}$ satisfy $J_{u_{n}} \subset \partial E_{n}$, we get $\sup _{n} \mathcal{H}^{d-1}\left(J_{u_{n}}\right)<$ $+\infty$. Moreover, by the growth assumptions on $f$ (see (2.1)) we get that $\left\|e\left(u_{n}\right)\right\|_{L^{p}(\Omega)}$ is uniformly bounded. Thus, by Theorem $3.5, u_{n}$ converges (up to a subsequence) weakly in $G S B D_{\infty}^{p}(\Omega)$ to some $u \in G S B D_{\infty}^{p}(\Omega)$. This concludes the proof.

We are now ready to prove Theorem 2.3.

Proof of Theorem 2.3. The existence of minimizers for $\bar{F}_{\mathrm{Dir}, \infty}$ follows by combining Theorem 5.8 and Theorem 5.9, by means of general properties of relaxations, see for example [26, Theorem 3.8]. To obtain minimizers for $\bar{F}_{\text {Dir }}$, it is enough to observe (recall (3.10), (3.12)) that

$$
\bar{F}_{\operatorname{Dir}, \infty}(u, E) \geqq \bar{F}_{\operatorname{Dir}}\left(v_{a}, E\right)
$$

for every $u \in G S B D_{\infty}^{p}(\Omega)$ and $v_{a}:=u \chi_{\Omega \backslash A_{u}^{\infty}}+a \chi_{A_{u}^{\infty}}\left(\right.$ recall $\left.A_{u}^{\infty}=\{u=\infty\}\right)$, where $a: \mathbb{R}^{d} \rightarrow \mathbb{R}^{d}$ is an arbitrary affine function with skew-symmetric gradient (usually called an infinitesimal rigid motion). Starting from a minimizer of $\bar{F}_{\text {Dir, } \infty \text {, }}$ if $A_{u}^{\infty} \neq \emptyset$, we thus obtain a family of minimizers for $\bar{F}_{\text {Dir }}$, parametrized by the infinitesimal rigid motions $a$. This concludes the proof.

\section{Functionals on Domains with a Subgraph Constraint}

In this section we prove the the results announced in Section 2.2.

\subsection{Relaxation of the Energy $G$}

This subsection is devoted to the proof of Theorem 2.4. The lower inequality is obtained by exploiting the tool of $\sigma_{\text {sym }}^{p}$-convergence introduced in Section 4 . The corresponding analysis will prove to be useful also for the compactness theorem in the next subsection. The proof of the upper inequality is quite delicate, and a careful procedure is needed to guarantee that the approximating displacements are still defined on a domain which is the subgraph of a function. We only follow partially the strategy in [20, Proposition 4.1], and employ also other arguments in order to improve the $G S B V$ proof which might fail in some pathological cases.

Consider a Lipschitz set $\omega \subset \mathbb{R}^{d-1}$ which is uniformly star-shaped with respect to the origin, see (2.9). We recall the notation $\Omega=\omega \times(-1, M+1)$ and

$$
\Omega_{h}=\left\{x \in \Omega:-1<x_{d}<h\left(x^{\prime}\right)\right\}, \quad \Omega_{h}^{+}=\Omega_{h} \cap\left\{x_{d}>0\right\}
$$

for $h: \omega \rightarrow[0, M]$ measurable, where we write $x=\left(x^{\prime}, x_{d}\right)$ for $x \in \mathbb{R}^{d}$. Moreover, we let $\Omega^{+}=\Omega \cap\left\{x_{d}>0\right\}$.

The lower inequality. Consider $\left(u_{n}, h_{n}\right)_{n}$ with $\sup _{n} G\left(u_{n}, h_{n}\right)<+\infty$. Then, we have that $h_{n} \in C^{1}(\omega ;[0, M]),\left.u_{n}\right|_{\Omega_{h_{n}}} \in W^{1, p}\left(\Omega_{h_{n}} ; \mathbb{R}^{d}\right),\left.u_{n}\right|_{\Omega \backslash \Omega_{h_{n}}}=0$, and $u_{n}=u_{0}$ on $\omega \times(-1,0)$. Suppose that $\left(u_{n}, h_{n}\right)_{n}$ converges in $L^{0}\left(\Omega ; \mathbb{R}^{d}\right) \times L^{1}(\omega)$ to $(u, h)$. We let

$$
\Gamma_{n}:=\partial \Omega_{h_{n}} \cap \Omega=\left\{x \in \Omega: h_{n}\left(x^{\prime}\right)=x_{d}\right\}
$$


be the graph of the function $h_{n}$. Note that $\sup _{n} \mathcal{H}^{d-1}\left(\Gamma_{n}\right)<+\infty$. We take $U=$ $\omega \times\left(-\frac{1}{2}, M\right)$ and $U^{\prime}=\Omega=\omega \times(-1, M+1)$, and apply Theorem 4.2, to deduce that $\left(\Gamma_{n}\right)_{n} \sigma_{\mathrm{sym}}^{p}$-converges (up to a subsequence) to a pair $\left(\Gamma, G_{\infty}\right)$. A fundamental step in the proof will be to show that

$$
G_{\infty}=\emptyset
$$

We postpone the proof of this property to Step 3 below. We first characterize the limiting set $\Gamma$ (Step 1) and prove the lower inequality (Step 2), by following partially the lines of [20, Section 3.2]. In the whole proof, for simplicity we omit to write $\tilde{\subset}$ and $\tilde{=}$ to indicate that the inclusions hold up to $\mathcal{H}^{d-1}$-negligible sets.

Step 1: Characterization of the limiting set $\Gamma$. Let us prove that the set

$$
\Sigma:=\Gamma \cap \Omega_{h}^{1}
$$

is vertical, that is

$$
\left(\Sigma+t e_{d}\right) \cap \Omega_{h}^{1} \subset \Sigma \text { for any } t \geqq 0 .
$$

This follows as in [20, Section 3.2]; in fact, consider $\left(v_{n}\right)_{n}$ and $v$ as in Definition 4.1(ii). In particular, $v_{n}=0$ on $U^{\prime} \backslash U, J_{v_{n}} \subset \Gamma_{n}$, and, in view of (6.3), $v$ is $\mathbb{R}^{d}$ valued with $\Gamma=J_{v}$. The functions $v_{n}^{\prime}(x):=v_{n}\left(x^{\prime}, x_{d}-t\right) \chi_{\Omega_{h_{n}}}(x)$ (with $t>0$, extended by zero in $\omega \times(-1,-1+t))$ converge to $v^{\prime}(x):=v\left(x^{\prime}, x_{d}-t\right) \chi_{\Omega_{h}}(x)$ in measure on $U^{\prime}$. Since $J_{v_{n}^{\prime}} \subset \Gamma_{n}$, Definition 4.1(i) implies $J_{v^{\prime}} \backslash \Gamma \subset\left(G_{\infty}\right)^{1}$. As $G_{\infty}=\emptyset$ by (6.3), we get $J_{v^{\prime}} \subset \Gamma$, so that

$\left(\Sigma+t e_{d}\right) \cap \Omega_{h}^{1}=\left(\Gamma+t e_{d}\right) \cap \Omega_{h}^{1}=\left(J_{v}+t e_{d}\right) \cap \Omega_{h}^{1}=J_{v^{\prime}} \cap \Omega_{h}^{1} \subset \Gamma \cap \Omega_{h}^{1}=\Sigma$,

where we have used $\Gamma=J_{v}$. This shows (6.5). In particular, $v_{\Sigma} \cdot e_{d}=0 \mathcal{H}^{d-1}$-a.e. in $\Sigma$. Next, we show that

$$
\mathcal{H}^{d-1}\left(\partial^{*} \Omega_{h} \cap \Omega\right)+2 \mathcal{H}^{d-1}(\Sigma) \leqq \liminf _{n \rightarrow \infty} \int_{\omega} \sqrt{1+\left|\nabla h_{n}\left(x^{\prime}\right)\right|^{2}} \mathrm{~d} x^{\prime} .
$$

To see this, we again consider functions $\left(v_{n}\right)_{n}$ and $v$ satisfying Definition 4.1(ii). In particular, we have $J_{v_{n}} \subset \Gamma_{n}$ and $J_{v}=\Gamma$. Since $\Gamma_{n}$ is the graph of a $C^{1}$ function, we either get $\left.v_{n}\right|_{\Omega_{h_{n}}} \equiv \infty$ or, by Korn's inequality, we have $\left.v_{n}\right|_{\Omega_{h_{n}}} \in$ $W^{1, p}\left(\Omega_{h_{n}} ; \mathbb{R}^{d}\right)$. Since $v_{n}=0$ on $U^{\prime} \backslash U$, we obtain $\left.v_{n}\right|_{\Omega_{h_{n}}} \in W^{1, p}\left(\Omega_{h_{n}} ; \mathbb{R}^{d}\right)$. We apply Theorem 5.1 for $E_{n}=\Omega \backslash \Omega_{h_{n}}, E=\Omega \backslash \Omega_{h}$, and the sequence of functions $w_{n}:=v_{n} \chi \Omega \backslash E_{n}=v_{n} \chi_{\Omega_{h_{n}}}$.

Observe that $\chi_{E_{n}} \rightarrow \chi_{E}$ in $L^{1}(\Omega)$. Moreover, $w_{n}$ converges weakly in $G S B D_{\infty}^{p}(\Omega)$ to $w:=v \chi_{\Omega_{h}}$ since $v_{n}$ converges weakly in $\operatorname{GSBD}_{\infty}^{p}(\Omega)$ to $v$ and $\sup _{n} \mathcal{H}^{d-1}\left(\partial E_{n}\right)<+\infty$. By $(5.2 \mathrm{~b})$ for $\varphi \equiv 1$ on $\mathbb{S}^{d-1}$ it holds that

$$
\mathcal{H}^{d-1}\left(\partial^{*} \Omega_{h} \cap \Omega\right)+2 \mathcal{H}^{d-1}\left(J_{w} \cap \Omega_{h}^{1}\right) \leqq \liminf _{n \rightarrow \infty} \mathcal{H}^{d-1}\left(\partial \Omega_{h_{n}} \cap \Omega\right),
$$

where we used that $E^{0}=\Omega_{h}^{1}$ and $\partial^{*} E \cap \Omega=\partial^{*} \Omega_{h} \cap \Omega$. Since $J_{v}=\Gamma$ and $J_{w} \cap \Omega_{h}^{1}=J_{v} \cap \Omega_{h}^{1}=\Sigma$, we indeed get (6.6), where for the right hand side we use that $\partial \Omega_{h_{n}}$ is the graph of the function $h_{n} \in C^{1}(\omega ;[0, M])$. For later purposes 
in Step 3, we also note that by Corollary 5.6 for $\phi(v)=|\xi \cdot v|$, with $\xi \in \mathbb{S}^{d-1}$ fixed, we get

$$
\begin{aligned}
\int_{\Gamma}\left|v_{\Gamma} \cdot \xi\right| \mathrm{d} \mathcal{H}^{d-1} & =\int_{J_{v}}\left|v_{v} \cdot \xi\right| \mathrm{d} \mathcal{H}^{d-1} \leqq \liminf _{n \rightarrow \infty} \int_{J_{v_{n}}}\left|v_{v_{n}} \cdot \xi\right| \mathrm{d} \mathcal{H}^{d-1} \\
& \leqq \liminf _{n \rightarrow \infty} \int_{\Gamma_{n}}\left|v_{\Gamma_{n}} \cdot \xi\right| \mathrm{d} \mathcal{H}^{d-1}
\end{aligned}
$$

(Strictly speaking, as $\phi$ is only a seminorm, we apply Corollary 5.6 for $\phi+\varepsilon$ for any $\varepsilon>0$.)

Step 2: The lower inequality. We now show the lower bound. Recall that $\left(u_{n}, h_{n}\right)_{n}$ converges in $L^{0}\left(\Omega ; \mathbb{R}^{d}\right) \times L^{1}(\omega)$ to $(u, h)$ and that $\left(G\left(u_{n}, h_{n}\right)\right)_{n}$ is bounded. Then, (2.1) and $\min _{\mathbb{S}^{d-1}} \varphi>0$ along with Theorem 3.5 and the fact that $\mathcal{L}^{d}(\{x \in$ $\left.\left.\Omega:\left|u_{n}(x)\right| \rightarrow \infty\right\}\right)=0$ imply that the limit $u=u \chi_{\Omega_{h}}$ lies in $\operatorname{GSBD}^{p}(\Omega)$. It also holds that $u=u_{0}$ on $\omega \times(-1,0)$ by (3.7)(i) and the fact that $u_{n}=u_{0}$ on $\omega \times(-1,0)$ for all $n \in \mathbb{N}$. In particular, we observe that $u_{n}=u_{n} \chi_{\Omega_{h_{n}}}$ converges weakly in $G S B D_{\infty}^{p}(\Omega)$ to $u$, cf. (3.16). The fact that $h \in B V(\omega ;[0, M])$ follows from a standard compactness argument. This shows $\bar{G}(u, h)<+\infty$.

To obtain the lower bound for the energy, we again apply Theorem 5.1 for $E_{n}=$ $\Omega \backslash \Omega_{h_{n}}$ and $E=\Omega \backslash \Omega_{h}$. Consider the sequence of functions $v_{n}:=\psi u_{n} \chi_{\Omega \backslash E_{n}}=$ $\psi u_{n}$, where $\psi \in C^{\infty}(\Omega)$ with $\psi=1$ in a neighborhood of $\Omega^{+}=\Omega \cap\left\{x_{d}>\right.$ $0\}$ and $\psi=0$ on $\omega \times\left(-1,-\frac{1}{2}\right)$. We observe that $v_{n}=0$ on $U^{\prime} \backslash U=\omega \times$ $\left(\left(-1,-\frac{1}{2}\right] \cup[M, M+1)\right)$ and that $v_{n}$ converges to $v:=\psi u \in G S B D^{p}(\Omega)$ weakly in $G S B D_{\infty}^{p}(\Omega)$. Now we apply Theorem 5.1. First, notice that (5.2a), $\psi=1$ on $\Omega^{+}$, and the fact that $A_{u}^{\infty}=\emptyset$ imply $e\left(u_{n}\right) \chi_{\Omega_{h_{n}}^{+}} \rightarrow e(u) \chi_{\Omega_{h}^{+}}$weakly in $L^{p}\left(\Omega ; \mathbb{M}_{\text {sym }}^{d \times d}\right)$. This along with the convexity of $f$ yields

$$
\int_{\Omega_{h}^{+}} f(e(u)) \mathrm{d} x \leqq \liminf _{n \rightarrow \infty} \int_{\Omega_{h_{n}}^{+}} f\left(e\left(u_{n}\right)\right) \mathrm{d} x .
$$

Moreover, applying Definition 4.1(i) on the sequence $\left(v_{n}\right)_{n}$, which satisfies $v_{n}=0$ on $U^{\prime} \backslash U$ and $J_{v_{n}} \subset \Gamma_{n}$, we observe $J_{u}=J_{v} \subset \Gamma$, where we have also used (6.3). Recalling the definition of $J_{u}^{\prime}=\left\{\left(x^{\prime}, x_{d}+t\right): x \in J_{u}, t \geqq 0\right\}$, see (2.10), and using (6.4)-(6.5) we find $J_{u}^{\prime} \cap \Omega_{h}^{1} \subset \Sigma$. Thus, by (6.6), we obtain

$$
\mathcal{H}^{d-1}\left(\partial^{*} \Omega_{h} \cap \Omega\right)+2 \mathcal{H}^{d-1}\left(J_{u}^{\prime} \cap \Omega_{h}^{1}\right) \leqq \liminf _{n \rightarrow \infty} \int_{\omega} \sqrt{1+\left|\nabla h_{n}\left(x^{\prime}\right)\right|^{2}} \mathrm{~d} x^{\prime} .
$$

Collecting (6.8) and (6.9) we conclude the lower inequality. To conclude the proof, it remains to confirm (6.3).

Step 3: Proof of $G_{\infty}=\emptyset$. Recall the definition of the graphs $\Gamma_{n}$ in (6.2) and its $\sigma_{\text {sym }}^{p}$-limit $\Gamma$ on the sets $U=\omega \times\left(-\frac{1}{2}, M\right)$ and $U^{\prime}=\Omega$. As before, consider $\psi \in C^{\infty}(\Omega)$ with $\psi=1$ in a neighborhood of $\Omega^{+}$and $\psi=0$ on $\omega \times\left(-1,-\frac{1}{2}\right)$. By employing (i) in Definition 4.1 for the sequence $v_{n}=\psi \chi \chi_{\Omega_{h_{n}}} e_{d}$ and its limit $v=\psi \chi_{\Omega_{h}} e_{d}$, we get that $\left(\partial^{*} \Omega_{h} \cap \Omega\right) \backslash \Gamma \subset\left(G_{\infty}\right)^{1}$. Since $U^{\prime} \cap \partial^{*} G_{\infty} \subset \Gamma$ by definition of $\sigma_{\text {sym }}^{p}$-convergence, we observe that

$$
\Gamma \supset\left(\partial^{*} G_{\infty} \cap \Omega\right) \cup\left(\partial^{*} \Omega_{h} \cap \Omega \cap\left(G_{\infty}\right)^{0}\right) .
$$




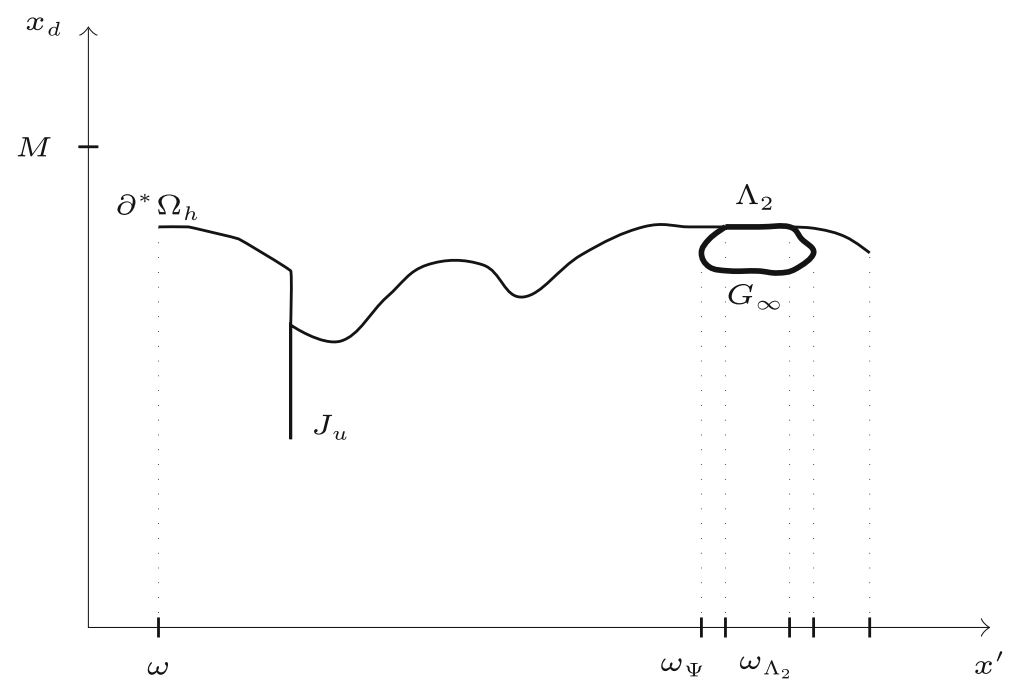

Fig. 1. A picture of the situation in the argument by contradiction. We show that in fact $G_{\infty}=\varnothing$

We estimate the $\mathcal{H}^{d-1}$-measure of the two terms on the right separately.

The first term. We define $\Psi=\partial^{*} G_{\infty} \cap \Omega$ for brevity. Since $G_{\infty}$ is contained in $U=$ $\omega \times\left(-\frac{1}{2}, M\right)$ and $\Omega=\omega \times(-1, M+1)$, we observe $\Psi=\partial^{*} G_{\infty} \cap(\omega \times \mathbb{R})$. Choose $\omega_{\Psi} \subset \omega$ such that $\omega_{\Psi} \times\{0\}$ is the orthogonal projection of $\Psi$ onto $\mathbb{R}^{d-1} \times\{0\}$. Note that $\Psi$ and $\omega_{\Psi}$ satisfy

$$
\mathcal{H}^{0}\left(\Psi_{y}^{e_{d}}\right) \geqq 2 \text { for all } y \in \omega_{\Psi} \times\{0\}
$$

since $G_{\infty}$ is a set of finite perimeter. Thus

$$
\int_{\omega \times\{0\}} \mathcal{H}^{0}\left(\left(\partial^{*} G_{\infty} \cap \Omega\right)_{y}^{e_{d}}\right) \mathrm{d} \mathcal{H}^{d-1}(y) \geqq 2 \mathcal{H}^{d-1}\left(\omega_{\Psi}\right) .
$$

The second term. As $\partial^{*} \Omega_{h} \cap \Omega$ is the (generalized) graph of the function $h: \omega \rightarrow$ $[0, M]$, we have

$$
\int_{\omega \times\{0\}} \mathcal{H}^{0}\left(\left(\partial^{*} \Omega_{h} \cap \Omega\right)_{y}^{e_{d}}\right) \mathrm{d} \mathcal{H}^{d-1}(y)=\mathcal{H}^{d-1}(\omega) .
$$

In a similar fashion, letting $\Lambda_{2}=\left(\partial^{*} \Omega_{h} \cap \Omega\right) \backslash\left(G_{\infty}\right)^{0}$ and denoting by $\omega_{\Lambda_{2}} \subset \omega$ its orthogonal projection onto $\mathbb{R}^{d-1} \times\{0\}$, we get

$$
\int_{\omega \times\{0\}} \mathcal{H}^{0}\left(\left(\left(\partial^{*} \Omega_{h} \cap \Omega\right) \backslash\left(G_{\infty}\right)^{0}\right)_{y}^{e_{d}}\right) \mathrm{d} \mathcal{H}^{d-1}(y)=\mathcal{H}^{d-1}\left(\omega_{\Lambda_{2}}\right) .
$$

As $\Lambda_{2}$ is contained in $\left(G_{\infty}\right)^{1} \cup \partial^{*} G_{\infty}$, we get $\omega_{\Lambda_{2}} \subset \omega_{\Psi}$, see Figure 1 . Therefore, by combining (6.12) and (6.13) we find 


$$
\begin{aligned}
& \int_{\omega \times\{0\}} \mathcal{H}^{0}\left(\left(\partial^{*} \Omega_{h} \cap \Omega \cap\left(G_{\infty}\right)^{0}\right)_{y}^{e_{d}}\right) \mathrm{d} \mathcal{H}^{d-1}(y)=\mathcal{H}^{d-1}(\omega)-\mathcal{H}^{d-1}\left(\omega_{\Lambda_{2}}\right) \\
& \geqq \mathcal{H}^{d-1}(\omega)-\mathcal{H}^{d-1}\left(\omega_{\Psi}\right) .
\end{aligned}
$$

Now (6.10), (6.11), (6.14) and the fact that $\partial^{*} G_{\infty} \cap\left(G_{\infty}\right)^{0}=\emptyset$ yield

$$
\begin{aligned}
\int_{\omega \times\{0\}} \mathcal{H}^{0}\left(\Gamma_{y}^{e_{d}}\right) \mathrm{d} \mathcal{H}^{d-1} \geqq & \int_{\omega \times\{0\}}\left(\mathcal{H}^{0}\left(\left(\partial^{*} G_{\infty} \cap \Omega\right)_{y}^{e_{d}}\right)\right. \\
& \left.+\mathcal{H}^{0}\left(\left(\partial^{*} \Omega_{h} \cap \Omega \cap\left(G_{\infty}\right)^{0}\right)_{y}^{e_{d}}\right)\right) \mathrm{d} \mathcal{H}^{d-1} \\
\geqq & \mathcal{H}^{d-1}(\omega)+\mathcal{H}^{d-1}\left(\omega_{\Psi}\right) .
\end{aligned}
$$

Since $\Gamma_{n}$ are graphs of the functions $h_{n}: \omega \rightarrow[0, M]$, we get by the area formula and (6.7) that

$\int_{\omega \times\{0\}} \mathcal{H}^{0}\left(\Gamma_{y}^{e_{d}}\right) \mathrm{d} \mathcal{H}^{d-1}(y)=\int_{\Gamma}\left|\nu_{\Gamma} \cdot e_{d}\right| \mathrm{d} \mathcal{H}^{d-1} \leqq \liminf _{n \rightarrow \infty} \int_{\Gamma_{n}}\left|v_{\Gamma_{n}} \cdot e_{d}\right| \mathrm{d} \mathcal{H}^{d-1}=\mathcal{H}^{d-1}(\omega)$.

This along with (6.15) shows that $\mathcal{H}^{d-1}\left(\omega_{\Psi}\right)=0$. By recalling that $\omega_{\Psi} \times\{0\}$ is the orthogonal projection of $\partial^{*} G_{\infty} \cap(\omega \times \mathbb{R})=\Psi$ onto $\mathbb{R}^{d-1} \times\{0\}$, we conclude that $G_{\infty}=\emptyset$.

This completes the proof of the lower inequality in Theorem 2.4.

The upper inequality. To obtain the upper inequality, it clearly suffices to prove the following result:

Proposition 6.1. Suppose that $f \geqq 0$ is convex and satisfies (2.1). Consider $(u, h)$ with $u=u \chi_{\Omega_{h}} \in G S B D^{p}(\Omega), u=u_{0}$ in $\omega \times(-1,0)$, and $h \in B V(\omega ;[0, M])$. Then, there exists a sequence $\left(u_{n}, h_{n}\right)_{n}$ with $h_{n} \in C^{1}(\omega) \cap B V(\omega ;[0, M]),\left.u_{n}\right|_{\Omega_{h_{n}}} \in$ $W^{1, p}\left(\Omega_{h_{n}} ; \mathbb{R}^{d}\right), u_{n}=0$ in $\Omega \backslash \Omega_{h_{n}}$, and $u_{n}=u_{0}$ in $\omega \times(-1,0)$ such that $u_{n} \rightarrow u$ in $L^{0}\left(\Omega ; \mathbb{R}^{d}\right), h_{n} \rightarrow h$ in $L^{1}(\omega)$, and

$$
\begin{aligned}
\limsup _{n \rightarrow \infty} \int_{\Omega_{h_{n}}} f\left(e\left(u_{n}\right)\right) \mathrm{d} x & \leqq \int_{\Omega_{h}} f(e(u)) \mathrm{d} x, \\
\lim \sup _{n \rightarrow \infty} \mathcal{H}^{d-1}\left(\partial \Omega_{h_{n}} \cap \Omega\right) & \leqq \mathcal{H}^{d-1}\left(\partial^{*} \Omega_{h} \cap \Omega\right)+2 \mathcal{H}^{d-1}\left(J_{u}^{\prime} \cap \Omega_{h}^{1}\right) .
\end{aligned}
$$

In particular, it is not restrictive to assume that $f \geqq 0$. In fact, otherwise we consider $\tilde{f}:=f+c_{2} \geqq 0$ changing the value of the elastic energy by the term $c_{2} \mathcal{L}^{d}\left(\Omega_{h}\right)$ which is continuous with respect to $L^{1}(\omega)$ convergence for $h$. Moreover, the integrals $\Omega_{h_{n}}$ and $\Omega_{h}$ can be replaced by $\Omega_{h_{n}}^{+}$and $\Omega_{h}^{+}$, respectively, since all functions coincide with $u_{0}$ on $\omega \times(-1,0)$.

Remark 6.2. The proof of the proposition will show that we can construct the sequence $\left(u_{n}\right)_{n}$ also in such a way that $u_{n} \in L^{\infty}\left(\Omega ; \mathbb{R}^{d}\right)$ holds for all $n \in \mathbb{N}$. This, however, comes at the expense of the fact that the boundary data is only satisfied approximately, that is, $\left.\left.u_{n}\right|_{\omega \times(-1,0)} \rightarrow u_{0}\right|_{\omega \times(-1,0)}$ in $W^{1, p}\left(\omega \times(-1,0) ; \mathbb{R}^{d}\right)$. This slightly different version will be instrumental in Section 6.3. 
As a preparation, we first state some auxiliary results. We recall two lemmas from [20]. The first is stated in [20, Lemma 4.3].

Lemma 6.3. Let $h \in B V(\omega ;[0,+\infty))$, with $\partial^{*} \Omega_{h}$ essentially closed, that is, $\mathcal{H}^{d-1}\left(\overline{\partial^{*} \Omega_{h}} \backslash \partial^{*} \Omega_{h}\right)=0$. Then, for any $\varepsilon>0$, there exists $g \in C^{\infty}(\omega ;[0,+\infty))$ such that $g \leqq h$ a.e. in $\omega,\|g-h\|_{L^{1}(\omega)} \leqq \varepsilon$, and

$$
\left|\int_{\omega} \sqrt{1+|\nabla g|^{2}} \mathrm{~d} x^{\prime}-\mathcal{H}^{d-1}\left(\partial^{*} \Omega_{h} \cap \Omega\right)\right| \leqq \varepsilon .
$$

Lemma 6.4. Let $h \in B V(\omega ;[0, M])$ and let $\Sigma \subset \mathbb{R}^{d}$ with $\mathcal{H}^{d-1}(\Sigma)<+\infty$ be vertical in the sense that $x=\left(x^{\prime}, x_{d}\right) \in \Sigma$ implies $\left(x^{\prime}, x_{d}+t\right) \in \Sigma$ as long as $\left(x^{\prime}, x_{d}+t\right) \in \Omega_{h}^{1}$. Then, for each $\varepsilon>0$ there exists $g \in C^{\infty}(\omega ;[0, M])$ such that

$$
\begin{aligned}
\|g-h\|_{L^{1}(\omega)} & \leqq \varepsilon, \\
\mathcal{H}^{d-1}\left(\left(\partial^{*} \Omega_{h} \cup \Sigma\right) \cap \Omega_{g}\right) & \leqq \varepsilon, \\
\left|\int_{\omega} \sqrt{1+|\nabla g|^{2}} \mathrm{~d} x^{\prime}-\left(\mathcal{H}^{d-1}\left(\partial^{*} \Omega_{h} \cap \Omega\right)+2 \mathcal{H}^{d-1}(\Sigma)\right)\right| & \leqq \varepsilon .
\end{aligned}
$$

Proof. We refer to the first step in the proof of [20, Proposition 4.1], in particular [20, Equation (12)-(13)]. We point out that the case of possibly unbounded graphs has been treated there, that is, $h \in B V(\omega ;[0,+\infty))$. The proof shows that the upper bound on $h$ is preserved and we indeed obtain $g \in C^{\infty}(\omega ;[0, M])$ if $h \in$ $B V(\omega ;[0, M])$.

Note that Lemma 6.4 states that $\partial^{*} \Omega_{h} \cup \Sigma$ can be approximated from below by a smooth graph $g$. However, this only holds up to a small portion, see (6.17b). Therefore, two additional approximation techniques are needed, one for graphs and one for GSBD functions. To this end, we introduce some notation which will also be needed for the proof of Proposition 6.1. Let $k \in \mathbb{N}, k>1$. For any $z \in\left(2 k^{-1}\right) \mathbb{Z}^{d}$, consider the hypercubes

$$
q_{z}^{k}:=z+\left(-k^{-1}, k^{-1}\right)^{d}, \quad Q_{z}^{k}:=z+\left(-5 k^{-1}, 5 k^{-1}\right)^{d} .
$$

Given an open set $U \subset \mathbb{R}^{d}$, we also define the union of cubes well contained in $U$ by

$$
(U)^{k}:=\operatorname{int}\left(\bigcup_{z: Q_{z}^{k} \subset U} \overline{q_{z}^{k}}\right)
$$

(Here, int (.) denotes the interior. This definition is unrelated to the notation $E^{s}$ for the set of points with density $s \in[0,1]$.)

We now address the two approximation results. We start by an approximation of graphs from which a union of hypercubes has been removed. Recall $\Omega^{+}=$ $\Omega \cap\left\{x_{d}>0\right\}$.

Lemma 6.5. Let $g \in C^{\infty}(\omega ;[0, M])$ and let $V_{k} \subset \Omega^{+}$be a union of cubes $Q_{z}^{k}$, $z \in \mathcal{Z} \subset\left(2 k^{-1}\right) \mathbb{Z}^{d}$, intersected with $\left(\Omega_{g}\right)^{k}$. Suppose that $V_{k}$ is vertical in the sense that $\left(x^{\prime}, x_{d}\right) \in V_{k}$ implies $\left(x^{\prime}, x_{d}+t\right) \in V_{k}$ for $t \geqq 0$ as long as $\left(x^{\prime}, x_{d}+t\right) \in\left(\Omega_{g}\right)^{k}$. 
Then, for $k \in \mathbb{N}$ sufficiently large, we find a function $h_{k} \in C^{\infty}(\omega ;[0, M])$ such that

$$
\begin{aligned}
& \mathcal{L}^{d}\left(\Omega_{g} \Delta \Omega_{h_{k}}\right) \leqq \mathcal{L}^{d}\left(\Omega_{g} \cap V_{k}\right)+C_{g, \omega} k^{-1} \\
& \mathcal{H}^{d-1}\left(\partial \Omega_{h_{k}} \cap \Omega\right) \leqq \mathcal{H}^{d-1}\left(\partial \Omega_{g} \cap \Omega\right)+\mathcal{H}^{d-1}\left(\partial V_{k} \cap\left(\Omega_{g}\right)^{k}\right)+C_{g, \omega} k^{-1}
\end{aligned}
$$

where $C_{g, \omega}>0$ depends on $d, g$, and $\omega$, but is independent of $k$. Moreover, there are constants $\tau_{g}, \tau_{*}>0$ only depending on $d, g$, and $\omega$ such that

$$
x=\left(x^{\prime}, x_{d}\right) \in \Omega_{h_{k}} \Rightarrow\left(\left(1-\tau_{*} / k\right) x^{\prime},\left(1-\tau_{*} / k\right) x_{d}-6 \tau_{g} / k\right) \in\left(\Omega_{g}\right)^{k} \backslash V_{k} .
$$

We point out that (6.21) means that $h_{k}$ lies below the boundary of $\left(\Omega_{g}\right)^{k} \backslash V_{k}$, up to a slight translation and dilation. We suggest to omit the proof of the lemma on first reading.

Proof. The proof relies on slight lifting and dilation of the set $\left(\Omega_{g}\right)^{k} \backslash V_{k}$ along with an application of Lemma 6.3. Recall definition (6.19), and define $\omega_{k} \subset \omega \subset \mathbb{R}^{d-1}$ such that $(\omega \times \mathbb{R})^{k}=\omega_{k} \times \mathbb{R}$. Since $\omega$ is uniformly star-shaped with respect to the origin, see (2.9), there exists a universal constant $\tau_{\omega}>0$ such that

$$
\omega_{k} \supset\left(1-\tau k^{-1}\right) \omega \text { for } \tau \geqq \tau_{\omega} .
$$

Define $\tau_{g}:=1+\sqrt{d} \max _{\omega}|\nabla g|$. For $k$ sufficiently large, it is elementary to check that

$$
\Omega_{g} \cap\left(\omega_{k} \times(0, \infty)\right) \subset\left(\left(\Omega_{g}\right)^{k}+6 \tau_{g} k^{-1} e_{d}\right) .
$$

We now "lift" the set $\left(\Omega_{g}\right)^{k} \backslash V_{k}$ upwards: define the function

$$
g_{k}^{\prime}\left(x^{\prime}\right):=\sup \left\{x_{d}<g\left(x^{\prime}\right):\left(x^{\prime}, x_{d}-6 \tau_{g} / k\right) \in\left(\Omega_{g}\right)^{k} \backslash V_{k}\right\} \quad \text { for } x^{\prime} \in \omega_{k} .
$$

We observe that $g_{k}^{\prime} \in B V\left(\omega_{k} ;[0, M]\right)$. Define $(\Omega)^{k}$ as in (6.19) and, similar to (6.1), we let $\Omega_{g_{k}^{\prime}}=\left\{x \in \omega_{k} \times(-1, M+1):-1<x_{d}<g_{k}^{\prime}\left(x^{\prime}\right)\right\}$. Since $V_{k}$ is vertical, we note that $\partial \Omega_{g_{k}^{\prime}} \cap(\Omega)^{k}$ is made of two parts: one part is contained in the smooth graph of $g$ and the rest in the boundary of $V_{k}+6 \tau_{g} k^{-1} e_{d}$. In particular, by (6.23) we get

$$
\begin{aligned}
\partial \Omega_{g_{k}^{\prime}} \cap & (\Omega)^{k} \subset\left(\partial \Omega_{g} \cap \Omega\right) \cup\left(\partial \Omega_{g_{k}^{\prime}} \cap(\Omega)^{k} \cap \Omega_{g}\right) \subset\left(\partial \Omega_{g} \cap \Omega\right) \cup\left(\left(\partial V_{k} \cap\left(\Omega_{g}\right)^{k}\right)\right. \\
& \left.+6 \tau_{g} k^{-1} e_{d}\right) .
\end{aligned}
$$

Then, we deduce

$$
\mathcal{H}^{d-1}\left(\partial \Omega_{g_{k}^{\prime}} \cap(\Omega)^{k}\right) \leqq \mathcal{H}^{d-1}\left(\partial \Omega_{g} \cap \Omega\right)+\mathcal{H}^{d-1}\left(\partial V_{k} \cap\left(\Omega_{g}\right)^{k}\right) .
$$

Since by (6.23) and (6.24) there holds $\left(\Omega_{g} \backslash \Omega_{g_{k}^{\prime}}\right) \cap(\Omega)^{k} \subset V_{k}+6 \tau_{g} k^{-1} e_{d}$, the fact that $V_{k}$ is vertical implies that

$$
\mathcal{L}^{d}\left(\left(\Omega_{g} \triangle \Omega_{g_{k}^{\prime}}\right) \cap(\Omega)^{k}\right) \leqq \mathcal{L}^{d}\left(\Omega_{g} \cap\left(V_{k}+6 \tau_{g} k^{-1} e_{d}\right)\right) \leqq \mathcal{L}^{d}\left(\Omega_{g} \cap V_{k}\right) .
$$


As $g_{k}^{\prime}$ is only defined on $\omega_{k}$, we further need a dilation: letting $\tau_{*}:=\tau_{\omega} \vee\left(6 \tau_{g}+6\right)$ and recalling (6.22) we define $g_{k}^{\prime \prime} \in B V(\omega ;[0, M])$ by

$$
g_{k}^{\prime \prime}\left(x^{\prime}\right)=g_{k}^{\prime}\left(\left(1-\tau_{*} k^{-1}\right) x^{\prime}\right) \text { for } x^{\prime} \in \omega .
$$

(The particular choice of $\tau_{*}$ will become clear in the proof of (6.21) below.) By (6.26) we get that

$$
\begin{aligned}
& \mathcal{L}^{d}\left(\Omega_{g} \Delta \Omega_{g_{k}^{\prime \prime}}\right) \leqq \mathcal{L}^{d}\left(\Omega_{g} \cap V_{k}\right)+C_{g, \omega} k^{-1}, \\
& \left|\mathcal{H}^{d-1}\left(\partial \Omega_{g_{k}^{\prime \prime}} \cap \Omega\right)-\mathcal{H}^{d-1}\left(\partial \Omega_{g_{k}^{\prime}} \cap(\Omega)^{k}\right)\right| \leqq C_{g, \omega} k^{-1},
\end{aligned}
$$

where the constant $C_{g, \omega}$ depends only on $d, g$, and $\omega$. We also notice that $\mathcal{H}^{d-1}$ $\left(\overline{\partial^{*} \Omega_{g_{k}^{\prime \prime}}} \backslash \partial^{*} \Omega_{g_{k}^{\prime \prime}}\right)=0$. Then by Lemma 6.3 applied for $\varepsilon=1 / k$ we find a function $h_{k} \in C^{\infty}(\omega ;[0, M])$ with $h_{k} \leqq g_{k}^{\prime \prime}$ on $\omega$ such that

$$
\left\|g_{k}^{\prime \prime}-h_{k}\right\|_{L^{1}(\omega)} \leqq k^{-1}, \quad\left|\mathcal{H}^{d-1}\left(\partial \Omega_{h_{k}} \cap \Omega\right)-\mathcal{H}^{d-1}\left(\partial^{*} \Omega_{g_{k}^{\prime \prime}} \cap \Omega\right)\right| \leqq k^{-1}
$$

By passing to a larger constant $C_{\omega, g}$ and by using (6.25), (6.28), and (6.29), we get (6.20). We finally show (6.21). In view of the definitions of $g_{k}^{\prime}$ and $g_{k}^{\prime \prime}$ in (6.24) and (6.27), respectively, and the fact that $h_{k} \leqq g_{k}^{\prime \prime}$, we get

$$
x=\left(x^{\prime}, x_{d}\right) \in \Omega_{h_{k}} \Rightarrow\left(\left(1-\tau_{*} / k\right) x^{\prime}, x_{d}-6 \tau_{g} / k\right) \in\left(\Omega_{g}\right)^{k} \backslash V_{k} .
$$

Recall $\tau_{*}=\tau_{\omega} \vee\left(6 \tau_{g}+6\right)$ and observe that $-\left(1-\tau_{*} / k\right)-6 \tau_{g} / k \geqq-1+6 / k$. Also note that $\left(\Omega_{g}\right)^{k} \supset(\Omega)^{k} \cap(\omega \times(-1+6 / k, 0))$, cf. (6.19). This, along with the verticality of $V_{k} \subset \Omega^{+}$, shows that

$x=\left(x^{\prime}, x_{d}\right) \in \Omega_{h_{k}} \Rightarrow\left(\left(1-\tau_{*} / k\right) x^{\prime},\left(1-\tau_{*} / k\right) x_{d}-6 \tau_{g} / k\right) \in\left(\Omega_{g}\right)^{k} \backslash V_{k}$.

This concludes the proof.

Next, we present an approximation technique for $G S B D$ functions based on [17]. In what follows, $\psi:[0, \infty) \rightarrow[0, \infty)$ denotes the function $\psi(t)=t \wedge 1$.

Lemma 6.6. Let $U \subset \mathbb{R}^{d}$ be open, bounded, $p>1$, and $k \in \mathbb{N}, \theta \in(0,1)$ with $k^{-1}, \theta$ small enough. Let $\mathcal{F} \subset G S B D^{p}(U)$ be such that $\psi(|v|)+|e(v)|^{p}$ is equiintegrable for $v \in \mathcal{F}$. Suppose that for $v \in \mathcal{F}$ there exists a set of finite perimeter $V \subset U$ such that for each $q_{z}^{k}, z \in\left(2 k^{-1}\right) \mathbb{Z}^{d}$, intersecting $(U)^{k} \backslash V$, it holds that

$$
\mathcal{H}^{d-1}\left(Q_{z}^{k} \cap J_{v}\right) \leqq \theta k^{1-d} .
$$

Then there exists a function $w_{k} \in W^{1, \infty}\left((U)^{k} \backslash V ; \mathbb{R}^{d}\right)$ such that

$$
\begin{aligned}
& \int_{(U)^{k} \backslash V} \psi\left(\left|w_{k}-v\right|\right) \mathrm{d} x \leqq R_{k}, \\
& \int_{(U)^{k} \backslash V}\left|e\left(w_{k}\right)\right|^{p} \mathrm{~d} x \leqq \int_{U}|e(v)|^{p} \mathrm{~d} x+R_{k},
\end{aligned}
$$

where $\left(R_{k}\right)_{k}$ is a sequence independent of $v \in \mathcal{F}$ with $R_{k} \rightarrow 0$ as $k \rightarrow \infty$. 
The lemma is essentially a consequence of the rough estimate proved in $[17$, Theorem 3.1]. For the convenience of the reader, we include a short proof in Appendix A.

After having collected auxiliary lemmas, we now give a short outline of the proof. Recall that $\Omega=\omega \times(-1, M+1)$ for given $M>0$. Consider a pair $(u, h)$ as in Proposition 6.1. We work with $u \chi_{\Omega_{h}} \in G S B D^{p}(\Omega)$ in the following, without specifying each time that $u=0$ in the complement of $\Omega_{h}$. Recall $J_{u}^{\prime}$ defined in (2.10), and, as before, set $\Sigma:=J_{u}^{\prime} \cap \Omega_{h}^{1}$. This implies $J_{u} \subset\left(\partial^{*} \Omega_{h} \cap \Omega\right) \cup \Sigma$. Since $\Sigma$ is vertical, we can approximate $\left(\partial^{*} \Omega_{h} \cap \Omega\right) \cup \Sigma$ by the graph of a smooth function $g \in C^{\infty}(\omega ;[0, M])$ in the sense of Lemma 6.4.

Our goal is to construct a regular approximation of $u$ in (most of) $\Omega_{g}$ by means of Lemma 6.6. The main step is to identify suitable exceptional sets $\left(V_{k}\right)_{k}$ such that for the cubes outside of $\left(V_{k}\right)_{k}$ we can verify (6.30). In this context, we emphasize that it is crucial that each $V_{k}$ is vertical since this allows us to apply Lemma 6.5 and to approximate the boundary of $\left(\Omega_{g}\right)^{k} \backslash V_{k}$ from below by a smooth graph. Before we start with the actual proof of Proposition 6.1, we address the construction of $\left(V_{k}\right)_{k}$. To this end, we introduce the notion of good and bad nodes, and collect some important properties.

Define the set of nodes

$$
\mathcal{N}_{k}:=\left\{z \in\left(2 k^{-1}\right) \mathbb{Z}^{d}: \overline{q_{z}^{k}} \subset \Omega_{g}\right\}
$$

Let us introduce the families of good nodes and bad nodes at level $k$. Let $\rho_{1}, \rho_{2}>0$ to be specified below. By $\mathcal{G}_{k}$ we denote the set of good nodes $z \in \mathcal{N}_{k}$, namely those satisfying

$$
\mathcal{H}^{d-1}\left(\overline{q_{z}^{k}} \cap\left(\partial^{*} \Omega_{h} \cup \Sigma\right)\right) \leqq \rho_{1} k^{1-d},
$$

or having the property that there exists a set of finite perimeter $F_{z}^{k} \subset q_{z}^{k}$, such that $q_{z}^{k} \cap \Omega_{h}^{0} \subset\left(F_{z}^{k}\right)^{1}, \quad \mathcal{H}^{d-1}\left(\partial^{*} F_{z}^{k}\right) \leqq \rho_{2} k^{1-d}, \quad \mathcal{H}^{d-1}\left(\overline{q_{z}^{k}} \cap \Sigma \cap\left(F_{z}^{k}\right)^{0}\right) \leqq \rho_{2} k^{1-d}$.

We define the set of bad nodes by $\mathcal{B}_{k}=\mathcal{N}_{k} \backslash \mathcal{G}_{k}$. Moreover, let

$$
\mathcal{G}_{k}^{*}:=\left\{z \in \mathcal{G}_{k}: \text { (6.33) does not hold }\right\} .
$$

For an illustration of the cubes in $\mathcal{G}_{k}$ we refer to Figure 2.

We partition the set of good nodes $\mathcal{G}_{k}$ into

$$
\mathcal{G}_{k}^{1}=\left\{z \in \mathcal{G}_{k}: \mathcal{L}^{d}\left(q_{z}^{k} \cap \Omega_{h}^{0}\right) \leqq \mathcal{L}^{d}\left(q_{z}^{k} \cap \Omega_{h}^{1}\right)\right\}, \quad \mathcal{G}_{k}^{2}=\mathcal{G}_{k} \backslash \mathcal{G}_{k}^{1} .
$$

We introduce the terminology " $q_{z^{\prime}}^{k}$ is above $q_{z}^{k}$ " meaning that $q_{z^{\prime}}^{k}$ and $q_{z}^{k}$ have the same vertical projection onto $\mathbb{R}^{d-1} \times\{0\}$ and $z_{d}^{\prime}>z_{d}$.

We remark that bad nodes have been defined differently in [20], namely as the cubes having an edge which intersects $\partial^{*} \Omega_{h} \cap \Sigma$. This definition is considerably easier than our definition. It may, however, fail in some pathological situations since, in this case, the union of cubes with bad nodes as centers does not necessarily form a "vertical set". 

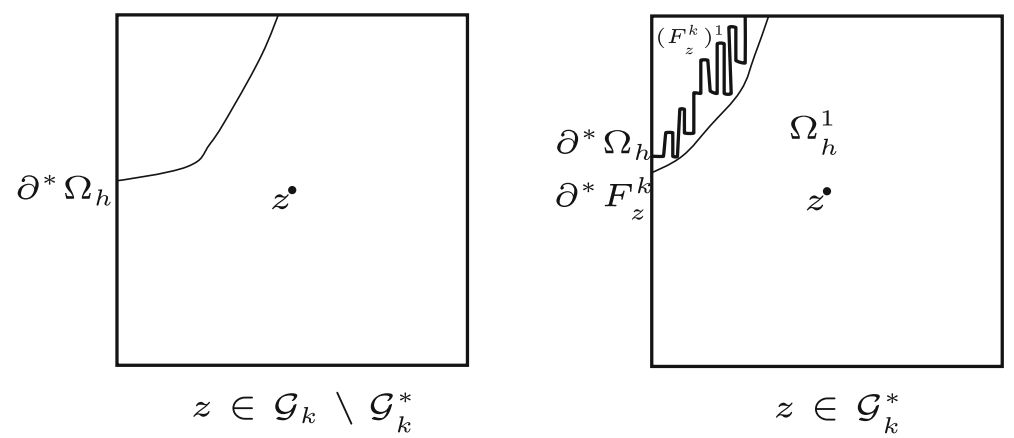

Fig. 2. A simplified representation of nodes in $\mathcal{G}_{k}$, for $d=2$ and with $\Sigma=\emptyset$. The set $\mathcal{G}_{k} \backslash \mathcal{G}_{k}^{*}$ corresponds to the cubes containing only a small portion of $\partial^{*} \Omega_{h} \cup \Sigma$, see first picture. For the cubes $\mathcal{G}_{k}^{*}$, the portion of $\partial^{*} \Omega_{h}$ is contained in a set $F_{z}^{k}$ with small boundary, see second picture. Intuitively, this along with the fact that (6.33) does not hold means that $\partial^{*} \Omega_{h}$ is highly oscillatory in such cubes

Lemma 6.7. (Properties of good and bad nodes) Given $\Omega_{h}$ and $\Sigma$, define $\Omega_{g}$ as in Lemma 6.4 for $\varepsilon>0$ sufficiently small. We can choose $0<\rho_{1}<\rho_{2}$ satisfying $\rho_{1}, \rho_{2} \leqq \frac{1}{2} 5^{-d} \theta$ such that the following properties hold for the good and bad nodes defined in (6.32)-(6.36):

(i) if $q_{z^{\prime}}^{k}$ is above $q_{z}^{k}$ and $z \in \mathcal{B}_{k} \cup \mathcal{G}_{k}^{2}$, then $z^{\prime} \in \mathcal{B}_{k} \cup \mathcal{G}_{k}^{2}$;

(ii) if $z, z^{\prime} \in \mathcal{G}_{k}$ with $\mathcal{H}^{d-1}\left(\partial q_{z}^{k} \cap \partial q_{z^{\prime}}^{k}\right)>0$, then $z, z^{\prime} \in \mathcal{G}_{k}^{1}$ or $z, z^{\prime} \in \mathcal{G}_{k}^{2}$;

(iii) $\# \mathcal{B}_{k}+\# \mathcal{G}_{k}^{*} \leqq 2 \rho_{1}^{-1} k^{d-1} \varepsilon$;

(iv) $\sum_{z \in \mathcal{G}_{k}^{2}} \mathcal{L}^{d}\left(\Omega_{h} \cap q_{z}^{k}\right) \leqq \varepsilon$.

We suggest to omit the proof of the lemma on first reading and to proceed directly with the proof of Proposition 6.1.

Proof. By $c_{\pi} \geqq 1$ we denote the maximum of the constants appearing in the isoperimetric inequality and the relative isoperimetric inequality on a cube in dimension $d$. We will show the statement for $\varepsilon$ and $0<\rho_{2}<1$ sufficiently small satisfying $\rho_{2} \leqq \frac{1}{2} 5^{-d} \theta$, and for $\rho_{1}=\left((3 d+1) c_{\pi}\right)^{-1} \rho_{2}$.

Preparations. First, we observe that for $\rho_{2}$ sufficiently small we have that $\mathcal{G}_{k}^{*} \subset$ $\mathcal{G}_{k}^{1}$. Indeed, since for $z \in \mathcal{G}_{k}^{*}$ property (6.34) holds, the isoperimetric inequality implies that

$$
\mathcal{L}^{d}\left(q_{z}^{k} \cap \Omega_{h}^{0}\right) \leqq \mathcal{L}^{d}\left(F_{z}^{k}\right) \leqq c_{\pi}\left(\mathcal{H}^{d-1}\left(\partial^{*} F_{z}^{k}\right)\right)^{d /(d-1)} \leqq c_{\pi} \rho_{2}^{d /(d-1)} k^{-d} .
$$

Then, for $\rho_{2}$ sufficiently small we get $\mathcal{L}^{d}\left(q_{z}^{k} \cap \Omega_{h}^{0}\right)<\frac{1}{2} \mathcal{L}^{d}\left(q_{z}^{k}\right)$, and thus $z \in \mathcal{G}_{k}^{1}$, see (6.36).

As a further preparation, we show that for each $z \in \mathcal{G}_{k}^{1}$ there exists a set of finite perimeter $H_{z}^{k}$ with $\Omega_{h}^{0} \cap q_{z}^{k} \subset H_{z}^{k} \subset q_{z}^{k}$ such that

$$
\mathcal{L}^{d}\left(H_{z}^{k}\right) \leqq c_{\pi} \rho_{2}^{d /(d-1)} k^{-d}, \quad \mathcal{H}^{d-1}\left(\partial^{*} H_{z}^{k}\right) \leqq \rho_{2} k^{1-d},
$$




$$
\mathcal{H}^{d-1}\left(\overline{q_{z}^{k}} \cap \Sigma \cap\left(H_{z}^{k}\right)^{0}\right) \leqq \rho_{2} k^{1-d} .
$$

Indeed, if (6.34) holds, this follows directly from (6.34) and (6.37) for $H_{z}^{k}:=F_{z}^{k}$.

Now suppose that $z \in \mathcal{G}_{k}^{1}$ satisfies (6.33). In this case, we define $H_{z}^{k}:=\Omega_{h}^{0} \cap q_{z}^{k}$. To control the volume, we use the relative isoperimetric inequality on $q_{z}^{k}$ to find by (6.33)

$$
\begin{aligned}
\mathcal{L}^{d}\left(H_{z}^{k}\right) & =\mathcal{L}^{d}\left(\Omega_{h}^{0} \cap q_{z}^{k}\right) \wedge \mathcal{L}^{d}\left(\Omega_{h}^{1} \cap q_{z}^{k}\right) \leqq c_{\pi}\left(\mathcal{H}^{d-1}\left(\partial^{*} \Omega_{h} \cap q_{z}^{k}\right)\right)^{d /(d-1)} \\
& \leqq c_{\pi} \rho_{1}^{d /(d-1)} k^{-d}
\end{aligned}
$$

that is, the first part of $(6.38)$ holds since $\rho_{1}=\left((3 d+1) c_{\pi}\right)^{-1} \rho_{2}$. To obtain the second estimate in (6.38), the essential step is to control $\mathcal{H}^{d-1}\left(\partial q_{z}^{k} \cap \Omega_{h}^{0}\right)$. For simplicity, we only estimate $\mathcal{H}^{d-1}\left(\partial_{d} q_{z}^{k} \cap \Omega_{h}^{0}\right)$ where $\partial_{d} q_{z}^{k}$ denotes the two faces of $\partial q_{z}^{k}$ whose normal vector is parallel to $e_{d}$. The other faces can be treated in a similar fashion. Write $z=\left(z^{\prime}, z_{d}\right)$ and define $\omega_{z}=z^{\prime}+\left(-k^{-1}, k^{-1}\right)^{d-1}$. By $\omega_{*} \subset \omega_{z}$ we denote the largest measurable set such that the cylindrical set $\left(\omega_{*} \times \mathbb{R}\right) \cap q_{z}^{k}$ is contained in $\Omega_{h}^{0}$. Then by the area formula (cf. for example [57, (12.4) in Section 12]) and by recalling notation (3.1) we get

$$
\begin{aligned}
\mathcal{H}^{d-1}\left(\partial_{d} q_{z}^{k} \cap \Omega_{h}^{0}\right) & \leqq 2 \mathcal{H}^{d-1}\left(\omega_{*}\right)+2 \int_{\left(\omega_{z} \backslash \omega_{*}\right) \times\{0\}} \mathcal{H}^{0}\left(\left(\partial^{*} \Omega_{h}\right)_{y}^{e_{d}}\right) \mathrm{d} \mathcal{H}^{d-1}(y) \\
& \leqq 2 \mathcal{H}^{d-1}\left(\omega_{*}\right)+2 \int_{\partial^{*} \Omega_{h} \cap q_{z}^{k}}\left|v_{\Omega_{h}} \cdot e_{d}\right| \mathrm{d} \mathcal{H}^{d-1} \\
& \leqq 2 \mathcal{H}^{d-1}\left(\omega_{*}\right)+2 \mathcal{H}^{d-1}\left(\partial^{*} \Omega_{h} \cap q_{z}^{k}\right)
\end{aligned}
$$

As $\left(\omega_{*} \times \mathbb{R}\right) \cap q_{z}^{k} \subset \Omega_{h}^{0} \cap q_{z}^{k}$ and $\mathcal{L}^{d}\left(\Omega_{h}^{0} \cap q_{z}^{k}\right) \leqq c_{\pi} \rho_{1}^{d /(d-1)} k^{-d}$ by (6.39), we deduce $2 k^{-1} \mathcal{H}^{d-1}\left(\omega_{*}\right) \leqq c_{\pi} \rho_{1}^{d /(d-1)} k^{-d}$. This along with (6.33) and (6.40), yields

$$
\mathcal{H}^{d-1}\left(\partial_{d} q_{z}^{k} \cap \Omega_{h}^{0}\right) \leqq c_{\pi} \rho_{1}^{d /(d-1)} k^{1-d}+2 \rho_{1} k^{1-d} \leqq 3 c_{\pi} \rho_{1} k^{1-d} .
$$

By repeating this argument for the other faces and by recalling $\mathcal{H}^{d-1}\left(\partial^{*} \Omega_{h} \cap q_{z}^{k}\right) \leqq$ $\rho_{1} k^{1-d}$, we conclude that $H_{z}^{k}=\Omega_{h}^{0} \cap q_{z}^{k}$ satisfies that

$$
\begin{aligned}
\mathcal{H}^{d-1}\left(\partial^{*} H_{z}^{k}\right) & =\mathcal{H}^{d-1}\left(\partial^{*} \Omega_{h} \cap q_{z}^{k}\right)+\mathcal{H}^{d-1}\left(\partial q_{z}^{k} \cap \Omega_{h}^{0}\right) \\
& \leqq \rho_{1} k^{1-d}+d \cdot 3 c_{\pi} \rho_{1} k^{1-d} \leqq \rho_{2} k^{1-d},
\end{aligned}
$$

where the last step follows from $\rho_{1}=\left((3 d+1) c_{\pi}\right)^{-1} \rho_{2}$. This concludes the the second part of (6.38). The third part follows from (6.33) and $\rho_{1} \leqq \rho_{2}$. We are now in a position to prove the statement.

Proof of $(i)$. We need to show that for $z^{\prime} \in \mathcal{G}_{k}^{1}$ there holds $z \in \mathcal{G}_{k}^{1}$ for all $z \in \mathcal{N}_{k}$ such that $q_{z^{\prime}}^{k}$ is above $q_{z}^{k}$. Fix such cubes $q_{z}^{k}$ and $q_{z^{\prime}}^{k}$.

Consider the set $H_{z^{\prime}}^{k}$ with $\Omega_{h}^{0} \cap q_{z^{\prime}}^{k} \subset H_{z^{\prime}}^{k} \subset q_{z^{\prime}}^{k}$ introduced in (6.38), and define $F_{z}^{k}:=H_{z^{\prime}}^{k}-z^{\prime}+z$. Since $\Omega_{h}$ is a generalized graph, we get $\left(F_{z}^{k}\right)^{1} \supset \Omega_{h}^{0} \cap q_{z}^{k}$. 
Moreover, since $\Sigma=J_{u}^{\prime} \cap \Omega_{h}^{1}$ is vertical in $\Omega_{h}$, see (2.10), and $\left(H_{z^{\prime}}^{k}\right)^{0} \subset \Omega_{h}^{1} \cap q_{z^{\prime}}^{k}$, we have

$$
\Sigma \cap\left(F_{z}^{k}\right)^{0}=\Sigma \cap \Omega_{h}^{1} \cap\left(F_{z}^{k}\right)^{0} \subset\left(\Sigma \cap \Omega_{h}^{1} \cap\left(H_{z^{\prime}}^{k}\right)^{0}\right)+z-z^{\prime}=\left(\Sigma \cap\left(H_{z^{\prime}}^{k}\right)^{0}\right)+z-z^{\prime} .
$$

By (6.38) we thus get $\mathcal{H}^{d-1}\left(\overline{q_{z}^{k}} \cap \Sigma \cap\left(F_{z}^{k}\right)^{0}\right) \leqq \mathcal{H}^{d-1}\left(\overline{q_{z^{\prime}}^{k}} \cap \Sigma \cap\left(H_{z^{\prime}}^{k}\right)^{0}\right) \leqq \rho_{2} k^{1-d}$. Then the third property in (6.34) is satisfied for $z$. Again by (6.38) we note that also the first two properties of (6.34) hold, and thus $z \in \mathcal{G}_{k}$. Using once more that $\Omega_{h}$ is a generalized graph, we get $\mathcal{L}^{d}\left(\Omega_{h} \cap q_{z}^{k}\right) \geqq \mathcal{L}^{d}\left(\Omega_{h} \cap q_{z^{\prime}}^{k}\right)$. Then $z^{\prime} \in \mathcal{G}_{k}^{1}$ implies $z \in \mathcal{G}_{k}^{1}$, see (6.36). This shows (i).

Proof of (ii). Suppose by contradiction that there exist $z \in \mathcal{G}_{k}^{1}$ and $z^{\prime} \in \mathcal{G}_{k}^{2}$ satisfying $\mathcal{H}^{d-1}\left(\partial q_{z}^{k} \cap \partial q_{z^{\prime}}^{k}\right)>0$. Define the set $F:=H_{z}^{k} \cup\left(\Omega_{h}^{0} \cap q_{z^{\prime}}^{k}\right)$ with $H_{z}^{k}$ from (6.38), and observe that $F$ is contained in the cuboid $q_{*}^{k}=\operatorname{int}\left(\overline{q_{z}^{k}} \cup \overline{q_{z^{\prime}}^{k}}\right)$. Since $H_{z}^{k} \supset \Omega_{h}^{0} \cap q_{z}^{k}$, we find

$$
\mathcal{H}^{d-1}\left(q_{*}^{k} \cap \partial^{*} F\right) \leqq \mathcal{H}^{d-1}\left(\partial^{*} H_{z}^{k}\right)+\mathcal{H}^{d-1}\left(\partial^{*} \Omega_{h} \cap q_{z^{\prime}}^{k}\right) .
$$

As $\mathcal{G}_{k}^{2} \cap \mathcal{G}_{k}^{*}=\emptyset$, cf. (6.37), for $z^{\prime} \in \mathcal{G}_{k}^{2}$ estimate (6.33) holds true. This along with (6.38) yields

$$
\mathcal{H}^{d-1}\left(q_{*}^{k} \cap \partial^{*} F\right) \leqq \rho_{2} k^{1-d}+\rho_{1} k^{1-d} \leqq 2 \rho_{2} k^{1-d} .
$$

Then, the relative isoperimetric inequality on $q_{*}^{k}$ yields

$$
\mathcal{L}^{d}\left(q_{*}^{k} \cap F\right) \wedge \mathcal{L}^{d}\left(q_{*}^{k} \backslash F\right) \leqq C_{*}\left(\mathcal{H}^{d-1}\left(q_{*}^{k} \cap \partial^{*} F\right)\right)^{d /(d-1)} \leqq C_{*}\left(2 \rho_{2}\right)^{d /(d-1)} k^{-d}
$$

for some universal $C_{*}>0$. On the other hand, it holds that $\mathcal{L}^{d}\left(q_{*}^{k} \cap F\right) \geqq \mathcal{L}^{d}\left(\Omega_{h}^{0} \cap\right.$ $\left.q_{z^{\prime}}^{k}\right) \geqq \frac{1}{2}\left(2 k^{-1}\right)^{d}$ and $\mathcal{L}^{d}\left(q_{*}^{k} \backslash F\right) \geqq \mathcal{L}^{d}\left(q_{z}^{k} \backslash H_{z}^{k}\right) \geqq\left(2 k^{-1}\right)^{d}-c_{\pi} \rho_{2}^{d /(d-1)} k^{-d}$ by (6.38). However, for $\rho_{2}$ sufficiently small, this contradicts (6.41). This concludes the proof of (ii).

Proof of (iii). Note that $\mathcal{H}^{d-1}$-a.e. point in $\mathbb{R}^{d}$ is contained in at most two different closed cubes $\overline{q_{z}^{k}}, \overline{q_{z^{\prime}}^{k}}$. Therefore, since the cubes with centers in $\mathcal{G}_{k}^{*}$ and $\mathcal{B}_{k}$ do not satisfy (6.33), we get

$$
\begin{aligned}
\# \mathcal{B}_{k}+\# \mathcal{G}_{k}^{*} & \leqq \rho_{1}^{-1} k^{d-1} \sum_{z \in \mathcal{B}_{k} \cup \mathcal{G}_{k}^{*}} \mathcal{H}^{d-1}\left(\overline{q_{z}^{k}} \cap\left(\partial^{*} \Omega_{h} \cup \Sigma\right)\right) \\
& \leqq 2 \rho_{1}^{-1} k^{d-1} \mathcal{H}^{d-1}\left(\left(\partial^{*} \Omega_{h} \cup \Sigma\right) \cap \Omega_{g}\right),
\end{aligned}
$$

where the last step follows from (6.32). This along with (6.17b) shows (iii).

Proof of (iv). Recall that each $z \in \mathcal{G}_{k}^{2}$ satisfies (6.33), cf. (6.35) and before (6.37). The relative isoperimetric inequality, (6.32) and (6.36) yield that

$$
\begin{aligned}
\sum_{z \in \mathcal{G}_{k}^{2}} \mathcal{L}^{d}\left(\Omega_{h} \cap q_{z}^{k}\right) & =\sum_{z \in \mathcal{G}_{k}^{2}} \mathcal{L}^{d}\left(\Omega_{h}^{0} \cap q_{z}^{k}\right) \wedge \mathcal{L}^{d}\left(\Omega_{h}^{1} \cap q_{z}^{k}\right) \\
& \leqq c_{\pi} \sum_{z \in \mathcal{G}_{k}^{2}}\left(\mathcal{H}^{d-1}\left(\partial^{*} \Omega_{h} \cap q_{z}^{k}\right)\right)^{\frac{d}{d-1}}
\end{aligned}
$$




$$
\begin{aligned}
& \leqq c_{\pi}\left(\sum_{z \in \mathcal{G}_{k}^{2}} \mathcal{H}^{d-1}\left(\partial^{*} \Omega_{h} \cap q_{z}^{k}\right)\right)^{d /(d-1)} \\
& \leqq c_{\pi}\left(\mathcal{H}^{d-1}\left(\partial^{*} \Omega_{h} \cap \Omega_{g}\right)\right)^{d /(d-1)} .
\end{aligned}
$$

By (6.17b) we conclude for $\varepsilon$ small enough that $\sum_{z \in \mathcal{G}_{k}^{2}} \mathcal{L}^{d}\left(\Omega_{h} \cap q_{z}^{k}\right) \leqq c_{\pi} \varepsilon^{d /(d-1)}$ $\leqq \varepsilon$.

Proof of Proposition 6.1. Consider a pair $(u, h)$ and set $\Sigma:=J_{u}^{\prime} \cap \Omega_{h}^{1}$ with $J_{u}^{\prime}$ as in (2.10). Given $\varepsilon>0$, we approximate $\left(\partial^{*} \Omega_{h} \cap \Omega\right) \cup \Sigma$ by the graph of a smooth function $g \in C^{\infty}(\omega ;[0, M])$ in the sense of Lemma 6.4. Define the good and bad nodes as in (6.32)-(6.36) for $0<\rho_{1}, \rho_{2} \leqq \frac{1}{2} 5^{-d} \theta$ such that the properties in Lemma 6.7 hold. We will first define approximating regular graphs (Step 1) and regular functions (Step 2) for fixed $\varepsilon>0$. Finally, we let $\varepsilon \rightarrow 0$ and obtain the result by a diagonal argument (Step 3 ). In the whole proof, $C>0$ will denote a constant depending only on $d, p, \rho_{1}$, and $\rho_{2}$.

Step 1: Definition of regular graphs. Recall (6.19). For each $k \in \mathbb{N}$, we define the set

$$
V_{k}:=\bigcup_{z \in \mathcal{G}_{k}^{2} \cup \mathcal{B}_{k}} Q_{z}^{k} \cap\left(\Omega_{g}\right)^{k} .
$$

We observe that

$$
\partial V_{k} \cap\left(\Omega_{g}\right)^{k} \subset \bigcup_{z \in \mathcal{B}_{k}} \partial Q_{z}^{k}
$$

In fact, consider $z \in \mathcal{B}_{k} \cup \mathcal{G}_{k}^{2}$ such that $Q_{z}^{k} \cap V_{k} \neq \varnothing$ and one face of $\partial Q_{z}^{k}$ intersects $\partial V_{k} \cap\left(\Omega_{g}\right)^{k}$. In view of (6.42), there exists an adjacent cube $q_{z^{\prime}}^{k}$ satisfying $\mathcal{H}^{d-1}\left(\partial q_{z}^{k} \cap \partial q_{z^{\prime}}^{k}\right)>0$ and $z^{\prime} \in \mathcal{G}_{k}^{1}$ since otherwise $\partial Q_{z}^{k} \cap \partial V_{k} \cap\left(\Omega_{g}\right)^{k}=\emptyset$. As $z^{\prime} \in \mathcal{G}_{k}^{1}$, Lemma 6.7(ii) implies $z \notin \mathcal{G}_{k}^{2}$ and therefore $z \in \mathcal{B}_{k}$. This shows (6.43). A similar argument yields

$$
V_{k}=\left(\bigcup_{z \in \mathcal{B}_{k}} Q_{z}^{k} \cup \bigcup_{z \in \mathcal{G}_{k}^{2}} q_{z}^{k}\right) \cap\left(\Omega_{g}\right)^{k}
$$

up to a negligible set. Indeed, since $V_{k}$ is a union of cubes of sidelength $2 k^{-1}$ centered in nodes in $\mathcal{N}_{k}$, it suffices to prove that for a fixed $z \in \mathcal{N}_{k} \cap V_{k}$ there holds (a) $z \in \mathcal{G}_{k}^{2}$ or that (b) there exists $z^{\prime} \in \mathcal{B}_{k}$ such that $z \in Q_{z^{\prime}}^{k}$. Arguing by contradiction, if $z \in \mathcal{N}_{k} \cap V_{k}$ and neither (a) nor (b) hold, we deduce that $z \in \mathcal{G}_{k}^{1}$ and $Q_{z}^{k} \cap \mathcal{B}_{k}=\emptyset$. Then all $z^{\prime} \in \mathcal{N}_{k} \cap Q_{z}^{k}$ lie in $\mathcal{G}_{k}$. More precisely, by $z \in \mathcal{G}_{k}^{1}$ and Lemma 6.7(ii) we get that all $z^{\prime} \in \mathcal{N}_{k} \cap Q_{z}^{k}$ lie in $\mathcal{G}_{k}^{1}$. Then $Q_{z}^{k} \cap\left(\mathcal{G}_{k}^{2} \cup \mathcal{B}_{k}\right)=\emptyset$, so that $q_{z}^{k} \cap V_{k}=\varnothing$ by (6.42). This contradicts $z \in V_{k}$.

Let us now estimate the surface and volume of $V_{k}$. By (6.43) and Lemma 6.7(iii) we get

$$
\mathcal{H}^{d-1}\left(\partial V_{k} \cap\left(\Omega_{g}\right)^{k}\right) \leqq \sum_{z \in \mathcal{B}_{k}} \mathcal{H}^{d-1}\left(\partial Q_{z}^{k}\right) \leqq C k^{1-d} \# \mathcal{B}_{k} \leqq C \varepsilon,
$$

where $C$ depends on $\rho_{1}$. In a similar fashion, by (6.44) and Lemma 6.7(iii),(iv) we obtain

$$
\mathcal{L}^{d}\left(V_{k} \cap \Omega_{h}\right) \leqq C k^{-d} \# \mathcal{B}_{k}+\sum_{z \in \mathcal{G}_{k}^{2}} \mathcal{L}^{d}\left(q_{z}^{k} \cap \Omega_{h}\right) \leqq C k^{-1} \varepsilon+\varepsilon \leqq C \varepsilon .
$$


Note that $V_{k}$ is vertical in the sense that $\left(x^{\prime}, x_{d}\right) \in V_{k}$ implies $\left(x^{\prime}, x_{d}+t\right) \in V_{k}$ for $t \geqq 0$ as long as $\left(x^{\prime}, x_{d}+t\right) \in\left(\Omega_{g}\right)^{k}$. This follows from Lemma 6.7(i) and (6.42).

We apply Lemma 6.5 for $g$ and $V_{k}$ to find functions $h_{k} \in C^{\infty}(\omega ;[0, M])$ satisfying (6.20) and (6.21). Therefore, by (6.17a), (6.20), and (6.46) we get

$$
\begin{aligned}
\mathcal{L}^{d}\left(\Omega_{h} \triangle \Omega_{h_{k}}\right) \leqq & \mathcal{L}^{d}\left(\Omega_{g} \triangle \Omega_{h_{k}}\right)+\mathcal{L}^{d}\left(\Omega_{g} \triangle \Omega_{h}\right) \leqq \mathcal{L}^{d}\left(\Omega_{g} \cap V_{k}\right) \\
& +C_{g, \omega} k^{-1}+\mathcal{L}^{d}\left(\Omega_{g} \triangle \Omega_{h}\right) \\
\leqq & \mathcal{L}^{d}\left(\Omega_{h} \cap V_{k}\right)+C_{g, \omega} k^{-1}+2 \mathcal{L}^{d}\left(\Omega_{g} \triangle \Omega_{h}\right) \leqq C \varepsilon+C_{g, \omega} k^{-1}
\end{aligned}
$$

Moreover, by (6.17c), (6.20) and (6.45) we obtain that

$$
\begin{aligned}
\mathcal{H}^{d-1}\left(\partial \Omega_{h_{k}} \cap \Omega\right) & \leqq \mathcal{H}^{d-1}\left(\partial \Omega_{g} \cap \Omega\right)+\mathcal{H}^{d-1}\left(\partial V_{k} \cap\left(\Omega_{g}\right)^{k}\right)+C_{g, \omega} k^{-1} \\
& \leqq \mathcal{H}^{d-1}\left(\partial^{*} \Omega_{h} \cap \Omega\right)+2 \mathcal{H}^{d-1}(\Sigma)+C \varepsilon+C_{g, \omega} k^{-1}
\end{aligned}
$$

Step 2: Definition of regular functions. Recall (6.34)-(6.35), and observe that Lemma 6.7(iii) implies

$\mathcal{L}^{d}\left(F^{k}\right) \leqq \sum_{z \in \mathcal{G}_{k}^{*}} \mathcal{L}^{d}\left(q_{z}^{k}\right) \leqq C k^{-d} \# \mathcal{G}_{k}^{*} \leqq C \varepsilon k^{-1}, \quad$ where $F^{k}:=\bigcup_{z \in \mathcal{G}_{k}^{*}}\left(F_{z}^{k}\right)^{1}$.

We define the functions $v_{k} \in G S B D^{p}(\Omega)$ by

$$
v_{k}:=u\left(1-\chi_{F^{k}}\right) \chi_{\Omega_{g}} .
$$

Since $u=0$ in $\Omega \backslash \Omega_{h}$ and $v_{k}=0$ in $\Omega \backslash \Omega_{g}$, we get by (6.17a) and (6.49)

$$
\limsup _{k \rightarrow \infty} \mathcal{L}^{d}\left(\left\{v_{k} \neq u\right\}\right) \leqq \limsup _{k \rightarrow \infty} \mathcal{L}^{d}\left(F^{k} \cup\left(\Omega_{h} \backslash \Omega_{g}\right)\right) \leqq C \varepsilon
$$

We also obtain

$$
\mathcal{H}^{d-1}\left(Q_{z}^{k} \cap J_{v_{k}}\right) \leqq \theta k^{1-d}
$$

for each $q_{z}^{k}$ intersecting $\left(\Omega_{g}\right)^{k} \backslash V_{k}$. To see this, note that the definitions of $\mathcal{N}_{k}$ in (6.32) and of $V_{k}$ in (6.42) imply that for each $q_{z}^{k}$ with $q_{z}^{k} \cap\left(\left(\Omega_{g}\right)^{k} \backslash V_{k}\right) \neq \emptyset$, each $z^{\prime} \in \mathcal{N}_{k}$ with $q_{z^{\prime}}^{k} \cap Q_{z}^{k} \neq \emptyset$ satisfies $z^{\prime} \in \mathcal{G}_{k}$. In view of $\rho_{1}<\rho_{2} \leqq \frac{1}{2} 5^{-d} \theta$ (see Lemma 6.7), the property then follows from (6.33), (6.34), $J_{u} \cap \Omega_{g} \subset \partial^{*} \Omega_{h} \cup \Sigma$, and the fact that $Q_{z}^{k}$ consists of $5^{d}$ different cubes $q_{z^{\prime}}^{k}$.

Notice that $\left|v_{k}\right| \leqq|u|$ and $\left|e\left(v_{k}\right)\right| \leqq|e(u)|$ pointwise a.e., that is, the functions $\psi\left(\left|v_{k}\right|\right)+\left|e\left(v_{k}\right)\right|^{p}$ are equiintegrable, where $\psi(t)=t \wedge 1$. In view of (6.51), we can apply Lemma 6.6 on $U=\Omega_{g}$ for the function $v_{k} \in G S B D^{p}\left(\Omega_{g}\right)$ and the sets $V_{k}$, to get functions $w_{k} \in W^{1, \infty}\left(\left(\Omega_{g}\right)^{k} \backslash V_{k} ; \mathbb{R}^{d}\right)$ such that (6.31a) and (6.31b) hold for a sequence $R_{k} \rightarrow 0$.

We now define the function $\hat{w}_{k}: \Omega \rightarrow \mathbb{R}^{d}$ by

$$
\hat{w}_{k}(x):= \begin{cases}w_{k}\left(\left(1-\tau_{*} / k\right) x^{\prime},\left(1-\tau_{*} / k\right) x_{d}-6 \tau_{g} / k\right) & \text { if }-1<x_{d}<h_{k}\left(x^{\prime}\right) \\ 0 & \text { otherwise }\end{cases}
$$


Note that, in view of (6.21), the mapping is well defined and satisfies $\left.\hat{w}_{k}\right|_{\Omega_{h_{k}}} \in$ $W^{1, \infty}\left(\Omega_{h_{k}} ; \mathbb{R}^{d}\right)$. By (6.17a) (6.31a), (6.47), (6.50), and $\psi \leqq 1$ we get

$$
\begin{gathered}
\limsup _{k \rightarrow \infty}\left\|\psi\left(\left|\hat{w}_{k}-u\right|\right)\right\|_{L^{1}(\Omega)} \leqq \limsup _{k \rightarrow \infty}\left(\left\|\psi\left(\left|\hat{w}_{k}-v_{k}\right|\right)\right\|_{L^{1}(\Omega)}\right. \\
\left.+\mathcal{L}^{d}\left(\left\{v_{k} \neq u\right\}\right)\right) \leqq C \varepsilon .
\end{gathered}
$$

In a similar fashion, by employing (6.31b) in place of (6.31a) and by the fact that $\left\|e\left(\hat{w}_{k}\right)\right\|_{L^{p}(\Omega)} \leqq\left(1+C_{M} k^{-1}\right)\left\|e\left(w_{k}\right)\right\|_{L^{p}\left(\left(\Omega_{g}\right)^{k} \backslash V_{k}\right)}$ for some $C_{M}$ depending on $M$ and $\tau_{*}$, we obtain

$$
\begin{aligned}
\limsup _{k \rightarrow \infty} \int_{\Omega}\left|e\left(\hat{w}_{k}\right)\right|^{p} \mathrm{~d} x & \leqq \limsup _{k \rightarrow \infty} \int_{\left(\Omega_{g}\right)^{k} \backslash V_{k}}\left|e\left(w_{k}\right)\right|^{p} \mathrm{~d} x \\
& \leqq \limsup _{k \rightarrow \infty} \int_{\Omega_{g}}\left|e\left(v_{k}\right)\right|^{p} \mathrm{~d} x \leqq \int_{\Omega_{h}}|e(u)|^{p} \mathrm{~d} x,
\end{aligned}
$$

where the last step follows from (6.49).

Step 3: Conclusion. Performing the construction above for $\varepsilon=1 / n, n \in \mathbb{N}$, and choosing for each $n \in \mathbb{N}$ an index $k=k(n) \in \mathbb{N}$ sufficiently large, we obtain a sequence $\left(\hat{w}_{n}, h_{n}\right)$ such that by (6.47) and (6.52) we get

$$
\hat{w}_{n} \rightarrow u=u \chi_{\Omega_{h}} \text { in } L^{0}\left(\Omega ; \mathbb{R}^{d}\right) \text { and } h_{n} \rightarrow h \text { in } L^{1}(\omega) .
$$

By (6.48) and the definition $\Sigma=J_{u}^{\prime} \cap \Omega_{h}^{1}$ we obtain (6.16b). By $G S B D^{p}$ compactness (see Theorem 3.5) applied on $\hat{w}_{n}=\hat{w}_{n} \chi_{\Omega_{h_{n}}} \in G S B D^{p}(\Omega)$ along with $\hat{w}_{n} \rightarrow u$ in $L^{0}\left(\Omega ; \mathbb{R}^{d}\right)$ we get

$$
\int_{\Omega_{h}}|e(u)|^{p} \mathrm{~d} x \leqq \liminf _{n \rightarrow \infty} \int_{\Omega_{h_{n}}}\left|e\left(\hat{w}_{n}\right)\right|^{p} \mathrm{~d} x .
$$

This along with (6.53) and the strict convexity of the norm $\|\cdot\|_{L^{p}(\Omega)}$ gives

$$
e\left(\hat{w}_{n}\right) \rightarrow e(u) \text { in } L^{p}\left(\Omega ; \mathbb{M}_{\mathrm{sym}}^{d \times d}\right) .
$$

In view of (2.1), this shows the statement apart from the fact that the configurations $\hat{w}_{n}$ do possibly not satisfy the boundary data. (that is, we have now proved the version described in Remark 6.2 since $\hat{w}_{n} \in L^{\infty}\left(\Omega ; \mathbb{R}^{d}\right)$.) It remains to adjust the boundary values.

To this end, choose a continuous extension operator from $W^{1, p}\left(\omega \times(-1,0) ; \mathbb{R}^{d}\right)$ to $W^{1, p}\left(\Omega ; \mathbb{R}^{d}\right)$ and denote by $\left(w_{n}\right)_{n}$ the extensions of $\left.\left(\hat{w}_{n}-u_{0}\right)\right|_{\omega \times(-1,0)}$ to $\Omega$. Clearly, $w_{n} \rightarrow 0$ strongly in $W^{1, p}\left(\Omega ; \mathbb{R}^{d}\right)$ since $\left.\left(\hat{w}_{n}-u_{0}\right)\right|_{\omega \times(-1,0)} \rightarrow 0$ in $W^{1, p}\left(\omega \times(-1,0) ; \mathbb{R}^{d}\right)$. We now define the sequence $\left(u_{n}\right)_{n}$ by $u_{n}:=\left(\hat{w}_{n}-\right.$ $\left.w_{n}\right) \chi_{\Omega_{h_{n}}}$. By (6.54) we immediately deduce $u_{n} \rightarrow u$ in $L^{0}\left(\Omega ; \mathbb{R}^{d}\right)$. Moreover, $\left.u_{n}\right|_{\Omega_{h_{n}}} \in W^{1, p}\left(\Omega_{h_{n}} ; \mathbb{R}^{d}\right), u_{n}=0$ in $\Omega \backslash \Omega_{h_{n}}, u_{n}=u_{0}$ a.e. in $\omega \times(-1,0)$ and (6.55) still holds with $u_{n}$ in place of $\hat{w}_{n}$. Due to (2.1), this shows (6.16a) and concludes the proof. 
Remark 6.8. (Volume constraint) Given a volume constraint $\mathcal{L}^{d}\left(\Omega_{h}^{+}\right)=m$ with $0<m<M \mathcal{H}^{d-1}(\omega)$, one can construct the sequence $\left(u_{n}, h_{n}\right)$ in Proposition 6.1 such that also $h_{n}$ satisfies the volume constraint, cf. [20, Remark 4.2]. Indeed, if $\|h\|_{\infty}<M$, we consider $h_{n}^{*}\left(x^{\prime}\right)=r_{n}^{-1} h_{n}\left(x^{\prime}\right)$ and $u_{n}^{*}\left(x^{\prime}, x_{d}\right)=u_{n}\left(x^{\prime}, r_{n} x_{d}\right)$, where $r_{n}:=m^{-1} \int_{\omega} h_{n} \mathrm{~d} x$. Then $\int_{\omega} h_{n}^{*} \mathrm{~d} x=m$. Note that we can assume $\left\|h_{n}\right\|_{\infty} \leqq\|h\|_{\infty}$ (apply Proposition 6.1 with $\|h\|_{\infty}$ in place of $M$ ). Since $r_{n} \rightarrow 1$, we then find $h_{n}: \omega \rightarrow[0, M]$ for $n$ sufficiently large, and (6.16) still holds.

If $\|h\|_{L^{\infty}(\omega)}=M$ instead, we need to perform a preliminary approximation: given $\delta>0$, define $\hat{h}^{\delta, M}=h \wedge(M-\delta)$ and $h_{\delta}\left(x^{\prime}\right)=r_{\delta}^{-1} \hat{h}^{\delta, M}\left(x^{\prime}\right)$, where $r_{\delta}=m^{-1} \int_{\omega} \hat{h}^{\delta, M} \mathrm{~d} x$. Since $\Omega_{h}$ is a subgraph and $m<M \mathcal{H}^{d-1}(\omega)$, it is easy to check that $r_{\delta}>(M-\delta) / M$ and therefore $\left\|h_{\delta}\right\|_{\infty}<M$. Moreover, by construction we have $\int_{\omega} h_{\delta} \mathrm{d} x=m$. We define $u_{\delta}\left(x^{\prime}, x_{d}\right)=u\left(x^{\prime}, r_{\delta} x_{d}\right) \chi_{\Omega_{h_{\delta}}}$. We now apply the above approximation on fixed $\left(u_{\delta}, h_{\delta}\right)$, then consider a sequence $\delta \rightarrow 0$, and use a diagonal argument.

Remark 6.9. (Surface tension) We remark that, similar to [9,20,35], we could also derive a relaxation result for more general models where the surface tension $\sigma_{S}$ for the substrate can be different from the the surface tension $\sigma_{C}$ of the crystal. This corresponds to surface energies of the form

$$
\sigma_{S} \mathcal{H}^{d-1}(\{h=0\})+\sigma_{C} \mathcal{H}^{d-1}\left(\partial \Omega_{h} \cap(\omega \times(0,+\infty))\right) .
$$

In the relaxed setting, the surface energy is then given by

$$
\left(\sigma_{S} \wedge \sigma_{C}\right) \mathcal{H}^{d-1}(\{h=0\})+\sigma_{C}\left(\mathcal{H}^{d-1}\left(\partial^{*} \Omega_{h} \cap(\omega \times(0,+\infty))\right)+2 \mathcal{H}^{d-1}\left(J_{u}^{\prime} \cap \Omega_{h}^{1}\right)\right) .
$$

We do not prove this fact here for simplicity, but refer to [20, Section 2.4, Remark 4.4] for details how the proof needs to be adapted to deal with such a situation.

\subsection{Compactness and Existence of Minimizers}

In this short subsection we give the proof of the compactness result stated in Theorem 2.5. As discussed in Section 2.2, this immediately implies the existence of minimizers for problem (2.11).

Proof of Theorem 2.5. Consider $\left(u_{n}, h_{n}\right)_{n}$ with $\sup _{n} G\left(u_{n}, h_{n}\right)<+\infty$. First, by (2.8) and a standard compactness argument we find $h \in B V(\omega ;[0, M])$ such that $h_{n} \rightarrow h$ in $L^{1}(\omega)$, up to a subsequence (not relabeled). Moreover, by (2.1), (2.8), and the fact that $J_{u_{n}} \subset \partial \Omega_{h_{n}} \cap \Omega$ we can apply Theorem 3.5 to obtain some $u \in G S B D_{\infty}^{p}(\Omega)$ such that $u_{n} \rightarrow u$ weakly in $G S B D_{\infty}^{p}$. We also observe that $u=u \chi \Omega_{h}$ and $u=u_{0}$ on $\omega \times(-1,0)$ by (3.7)(i), $u_{n}=u_{n} \chi_{\Omega_{h_{n}}}$, and $u_{n}=u_{0}$ on $\omega \times(-1,0)$ for all $n \in \mathbb{N}$. It remains to show that $u \in G S B D^{p}(\Omega)$, that is, $\{u=\infty\}=\emptyset$.

To this end, we take $U=\omega \times\left(-\frac{1}{2}, M\right)$ and $U^{\prime}=\Omega=\omega \times(-1, M+1)$, and apply Theorem 4.2 on the sequence $\Gamma_{n}=\partial \Omega_{h_{n}} \cap \Omega$ to find that $\Gamma_{n} \sigma_{\text {sym }}^{p}$-converges (up to a subsequence) to a pair $\left(\Gamma, G_{\infty}\right)$. Consider $v_{n}=\psi u_{n}$, where $\psi \in C^{\infty}(\Omega)$ with $\psi=1$ in a neighborhood of $\omega \times(0, M+1)$ and $\psi=0$ on $\omega \times\left(-1,-\frac{1}{2}\right)$. 
Clearly, $v_{n}$ converges weakly in $\operatorname{SSBD}_{\infty}^{p}(\Omega)$ to $v:=\psi u$. As $J_{v_{n}} \subset \Gamma_{n}$ and $v_{n}=0$ on $U^{\prime} \backslash U$ for all $n \in \mathbb{N}$, we also obtain $\{v=\infty\} \subset G_{\infty}$ (up to a $\mathcal{L}^{d}$-negligible set), see Definition 4.1(i). As by definition of $v$ we have $\{u=\infty\}=\{v=\infty\}$, we deduce $\{u=\infty\} \subset G_{\infty}$. It now suffices to recall $G_{\infty}=\emptyset$, see (6.3), to conclude $\{u=\infty\}=\emptyset$.

\subsection{Phase Field Approximation of $\bar{G}$}

This final subsection is devoted to the phase-field approximation of the functional $\bar{G}$. Recall the functionals introduced in (2.12).

Proof of Theorem 2.6. Fix a decreasing sequence $\left(\varepsilon_{n}\right)_{n}$ of positive numbers converging to zero. We first prove the liminf and then the limsup inequality.

Proof of $(i)$. Let $\left(u_{n}, v_{n}\right)_{n}$ with $\sup _{n} G_{\varepsilon_{n}}\left(u_{n}, v_{n}\right)<+\infty$. Then, $v_{n}$ is nonincreasing in $x_{d}$, and therefore

$$
\widetilde{v}_{n}(x):=0 \vee\left(v_{n}(x)-\delta_{n} x_{d}\right) \wedge 1 \text { for } x \in \Omega=\omega \times(-1, M+1)
$$

is strictly decreasing on $\left\{0<\widetilde{v}_{n}<1\right\}$, where $\left(\delta_{n}\right)_{n}$ is a decreasing sequence of positive numbers converging to zero. For a suitable choice of $\left(\delta_{n}\right)_{n}$, depending on $\left(\varepsilon_{n}\right)_{n}$ and $W$, we obtain $\left\|v_{n}-\widetilde{v}_{n}\right\|_{L^{1}(\Omega)} \rightarrow 0$ and

$$
G_{\varepsilon_{n}}\left(u_{n}, v_{n}\right)=G_{\varepsilon_{n}}\left(u_{n}, \widetilde{v}_{n}\right)+O(1 / n) .
$$

By using the implicit function theorem and the coarea formula for $\widetilde{v}_{n}$, we can see, exactly as in the proof of [20, Theorem 5.1], that for a.e. $s \in(0,1)$ and $n \in \mathbb{N}$ the superlevel set $\left\{\widetilde{v}_{n}>s\right\}$ is the subgraph of a function $h_{n}^{s} \in H^{1}(\omega ;[0, M])$. (Every $h_{n}^{s}$ takes values in $[0, M]$ since $\widetilde{v}_{n}=0$ in $\omega \times(M, M+1)$.) By the coarea formula for $\widetilde{v}_{n}, \partial^{*}\left\{\widetilde{v}_{n}>s\right\} \cap \Omega=\partial^{*} \Omega_{h_{n}^{s}} \cap \Omega$, and Young's inequality we obtain that

$$
\begin{aligned}
& \int_{0}^{1} \sqrt{2 W(s)} \mathcal{H}^{d-1}\left(\partial^{*} \Omega_{h_{n}^{s}} \cap \Omega\right) \mathrm{d} s \quad \leqq \int_{\Omega} \sqrt{2 W\left(\widetilde{v}_{n}\right)}\left|\nabla \widetilde{v}_{n}\right| \mathrm{d} x \\
& \quad \leqq \int_{\Omega}\left(\frac{\varepsilon_{n}}{2}\left|\nabla \widetilde{v}_{n}\right|^{2}+\frac{1}{\varepsilon_{n}} W\left(\widetilde{v}_{n}\right)\right) \mathrm{d} x .
\end{aligned}
$$

Then, by Fatou's lemma, we get that

$$
\begin{aligned}
& \int_{0}^{1} \sqrt{2 W(s)}\left(\liminf _{n \rightarrow \infty} \int_{\omega} \sqrt{1+\left|\nabla h_{n}^{s}\left(x^{\prime}\right)\right|^{2}} \mathrm{~d} x^{\prime}\right) \mathrm{d} s \\
& \leq \liminf _{n \rightarrow \infty} \int_{\Omega}\left(\frac{\varepsilon_{n}}{2}\left|\nabla \widetilde{v}_{n}\right|^{2}+\frac{1}{\varepsilon_{n}} W\left(\widetilde{v}_{n}\right)\right) \mathrm{d} x<+\infty
\end{aligned}
$$

and thus $\liminf _{n \rightarrow \infty} \int_{\omega} \sqrt{1+\left|\nabla h_{n}^{s}\left(x^{\prime}\right)\right|^{2}} \mathrm{~d} x^{\prime}$ is finite for a.e. $s \in(0,1)$. By a diagonal argument, we can find a subsequence (still denoted by $\left.\left(\varepsilon_{n}\right)_{n}\right)$ and $\left(s_{k}\right)_{k} \subset$ $(0,1)$ with $\lim _{k \rightarrow \infty} s_{k}=0$ such that for every $k \in \mathbb{N}$ it holds that

$$
\lim _{n \rightarrow \infty} \int_{\omega} \sqrt{1+\left|\nabla h_{n}^{s_{k}}\left(x^{\prime}\right)\right|^{2}} \mathrm{~d} x^{\prime}=\liminf _{n \rightarrow \infty} \int_{\omega} \sqrt{1+\left|\nabla h_{n}^{s_{k}}\left(x^{\prime}\right)\right|^{2}} \mathrm{~d} x^{\prime}<+\infty .
$$


Up to a further (not relabeled) subsequence, we may thus assume that $h_{n}^{s_{k}}$ converges in $L^{1}(\omega)$ to some function $h^{s_{k}}$ for every $k$. Since $\sup _{n} G_{\varepsilon_{n}}\left(u_{n}, \widetilde{v}_{n}\right)<+\infty$ and thus $W\left(\widetilde{v}_{n}\right) \rightarrow 0$ a.e. in $\Omega$, we obtain $\widetilde{v}_{n} \rightarrow 0$ for a.e. $x$ with $x_{d}>h^{s_{k}}\left(x^{\prime}\right)$ and $\tilde{v}_{n} \rightarrow 1$ for a.e. $x$ with $x_{d}<h^{s_{k}}\left(x^{\prime}\right)$. (Recall $W(t)=0 \Leftrightarrow t \in\{0,1\}$.) This shows that the functions $h^{s_{k}}$ are independent of $k$, and will be denoted simply by $h \in B V(\omega ;[0, M])$.

Let us denote by $u_{n}^{k} \in G S B D^{p}(\Omega)$ the function given by

$$
u_{n}^{k}(x)= \begin{cases}u_{n}(x) & \text { if } x_{d}<h_{n}^{s_{k}}\left(x^{\prime}\right) \\ 0 & \text { else. }\end{cases}
$$

Then $\left(u_{n}^{k}\right)_{n}$ satisfies the hypothesis of Theorem 3.5 for every $k \in \mathbb{N}$. Indeed, $J_{u_{n}^{k}} \subset$ $\partial^{*} \Omega_{h_{n}^{s_{k}}}$ and $\mathcal{H}^{d-1}\left(\partial^{*} \Omega_{h_{n}^{s_{k}}}\right)$ is uniformly bounded in $n$ by (6.58). Moreover, $\left(e\left(u_{n}^{k}\right)\right)_{n}$ is uniformly bounded in $L^{p}\left(\Omega ; \mathbb{M}_{\text {sym }}^{d \times d}\right)$ by (2.1) and the fact that

$$
G_{\varepsilon_{n}}\left(u_{n}, \widetilde{v}_{n}\right) \geqq\left(\eta_{\varepsilon_{n}}+s_{k}^{2}\right) \int_{\Omega} f\left(e\left(u_{n}^{k}\right)\right) \mathrm{d} x .
$$

Therefore, Theorem 3.5 implies that, up to a subsequence, $u_{n}^{k}$ converges weakly in $G S B D_{\infty}^{p}(\Omega)$ to a function $u^{k}$. Furthermore, we infer, arguing exactly as in the proof of Theorem 2.5 above, that actually $u^{k} \in G S B D^{p}(\Omega)$, that is, the exceptional set $\left\{u^{k}=\infty\right\}$ is empty. By (3.7)(i) this yields $u_{n}^{k} \rightarrow u^{k}$ in $L^{0}\left(\Omega ; \mathbb{R}^{d}\right)$. By a diagonal argument we get (up to a further subsequence) that $u_{n}^{k} \rightarrow u^{k}$ pointwise a.e. as $n \rightarrow \infty$ for all $k \in \mathbb{N}$.

Recalling now the definition of $u_{n}^{k}$ in (6.59) and the fact that $\lim _{n \rightarrow \infty} \| h_{n}^{s_{k}}-$ $h \|_{L^{1}(\omega)}=0$ for all $k \in \mathbb{N}$, we deduce that the functions $u^{k}$ are independent of $k$. This function will simply be denoted by $u \in G S B D^{p}(\Omega)$ in the following. Note that $u=u \chi_{\Omega_{h}}$ and that $u=u_{0}$ on $\omega \times(-1,0)$ since $u_{n}=u_{0}$ on $\omega \times(-1,0)$ for all $n \in \mathbb{N}$.

For the proof of (2.13), we can now follow exactly the lines of the lower bound in [20, Theorem 5.1]. We sketch the main arguments for convenience of the reader. We first observe that

$$
\begin{aligned}
\int_{\Omega} \widetilde{v}_{n} f\left(e\left(u_{n}\right)\right) \mathrm{d} x & =\int_{\Omega}\left(2 \int_{0}^{\widetilde{v}_{n}(x)} s \mathrm{~d} s\right) f\left(e\left(u_{n}\right)(x)\right) \mathrm{d} x \\
& \geqq \int_{0}^{1} 2 s\left(\int_{\left\{\widetilde{v}_{n}>s\right\}} f\left(e\left(u_{n}\right)\right) \mathrm{d} x\right) \mathrm{d} s .
\end{aligned}
$$

This, along with (6.57) and Fatou's lemma, yields that

$$
\begin{aligned}
& \int_{0}^{1} \liminf _{n \rightarrow \infty}\left(2 s \int_{\left\{\widetilde{v}_{n}>s\right\}} f\left(e\left(u_{n}\right)\right) \mathrm{d} x+c_{W} \sqrt{2 W(s)} \int_{\omega} \sqrt{1+\left|\nabla h_{n}^{s}\right|^{2}} \mathrm{~d} x^{\prime}\right) \mathrm{d} s \\
& \quad \leqq \liminf _{n \rightarrow \infty} G_{\varepsilon_{n}}\left(u_{n}, \widetilde{v}_{n}\right) .
\end{aligned}
$$

Thus, the integrand

$$
I_{n}^{s}:=2 s \int_{\left\{\widetilde{v}_{n}>s\right\}} f\left(e\left(u_{n}\right)\right) \mathrm{d} x+c_{W} \sqrt{2 W(s)} \int_{\omega} \sqrt{1+\left|\nabla h_{n}^{s}\right|^{2}} \mathrm{~d} x^{\prime}
$$


is finite for a.e. $s \in(0,1)$. We then take $s$ such that $h_{n}^{s} \in H^{1}(\omega)$ for all $n$, and consider a subsequence $\left(n_{m}\right)_{m}$ such that $\lim _{m \rightarrow \infty} I_{n_{m}}^{s}=\liminf _{n \rightarrow \infty} I_{n}^{s}$. Exactly as in (6.59), we let $u_{n_{m}}^{s}$ be the function given by $u_{n_{m}}$ if $x_{d}<h_{n_{m}}^{s}\left(x^{\prime}\right)$ and by zero otherwise. Repeating the compactness argument below (6.59), we get $u_{n_{m}}^{s} \rightarrow u$ a.e. in $\Omega$ and $h_{n_{m}}^{s} \rightarrow h$ in $L^{1}(\omega)$ as $m \rightarrow \infty$. We observe that this can be done for a.e. $s \in(0,1)$, for a subsequence depending on $s$.

By $\int_{\left\{\widetilde{v}_{n_{m}}>s\right\}} f\left(e\left(u_{n_{m}}\right)\right) \mathrm{d} x=\int_{\Omega} f\left(e\left(u_{n_{m}}^{s}\right)\right) \mathrm{d} x$ and the (lower inequality in the) relaxation result Theorem 2.4 (up to different constants in front of the elastic energy and surface energy) we obtain

$$
\begin{aligned}
& 2 s \int_{\Omega_{h}^{+}} f(e(u)) \mathrm{d} x+c_{W} \sqrt{2 W(s)}\left(\mathcal{H}^{d-1}\left(\partial^{*} \Omega_{h} \cap \Omega\right)+2 \mathcal{H}^{d-1}\left(J_{u}^{\prime} \cap \Omega_{h}^{1}\right)\right) \\
& \quad \leqq \lim _{n_{m} \rightarrow \infty} I_{n_{m}}^{s}=\liminf _{n \rightarrow \infty} I_{n}^{s}
\end{aligned}
$$

for a.e. $s \in(0,1)$. We obtain (2.13) by integrating the above inequality and by using (6.56) and (6.60). Indeed, the integral on the left-hand side gives exactly $\bar{G}(u, h)$ as $c_{W}=\left(\int_{0}^{1} \sqrt{2 W(s)} \mathrm{d} s\right)^{-1}$.

Proof of $(i i)$. Let $(u, h)$ with $\bar{G}(u, h)<+\infty$. By the construction in the upper inequality for Theorem 2.4, see Proposition 6.1 and Remark 6.2, we find $h_{n} \in$ $C^{1}(\omega ;[0, M])$ with $h_{n} \rightarrow h$ in $L^{1}(\omega)$ and $u_{n} \in L^{\infty}\left(\Omega ; \mathbb{R}^{d}\right)$ with $\left.u_{n}\right|_{\Omega_{h_{n}}} \in$ $W^{1, p}\left(\Omega_{h_{n}} ; \mathbb{R}^{d}\right)$ and $u_{n} \rightarrow u$ a.e. in $\Omega$ such that

$\bar{G}(u, h)=\lim _{n \rightarrow \infty} H\left(u_{n}, h_{n}\right) \quad$ for $H\left(u_{n}, h_{n}\right):=\int_{\Omega_{h_{n}^{+}}^{+}} f\left(e\left(u_{n}\right)\right) \mathrm{d} x+\mathcal{H}^{d-1}\left(\partial \Omega_{h_{n}} \cap \Omega\right)$,

as well as

$$
\left.\left(u_{n}-u_{0}\right)\right|_{\omega \times(-1,0)} \rightarrow 0 \text { in } W^{1, p}\left(\omega \times(-1,0) ; \mathbb{R}^{d}\right) .
$$

For each $\left(u_{n}, h_{n}\right)$, we can use the construction in [20] to find sequences $\left(u_{n}^{k}\right)_{k} \subset$ $W^{1, p}\left(\Omega ; \mathbb{R}^{d}\right)$ and $\left(v_{n}^{k}\right)_{k} \subset H^{1}(\Omega ;[0,1])$ with $u_{n}^{k}=u_{n}$ on $\omega \times(-1,0), u_{n}^{k} \rightarrow u_{n}$ in $L^{1}\left(\Omega ; \mathbb{R}^{d}\right)$, and $v_{n}^{k} \rightarrow \chi_{\Omega_{h_{n}}}$ in $L^{1}(\Omega)$ such that (cf. (6.61))

$\limsup _{k \rightarrow \infty} \int_{\Omega}\left(\left(\left(v_{n}^{k}\right)^{2}+\eta_{\varepsilon_{k}}\right) f\left(e\left(u_{n}^{k}\right)\right)+c_{W}\left(\frac{W\left(v_{n}^{k}\right)}{\varepsilon_{k}}+\frac{\varepsilon_{k}}{2}\left|\nabla v_{n}^{k}\right|^{2}\right)\right) \mathrm{d} x \leqq H\left(u_{n}, h_{n}\right)$.

In particular, we refer to [20, Equation (28)] and mention that the functions $\left(v_{n}^{k}\right)_{k}$ can be constructed such that $v_{n}^{k}=1$ on $\omega \times(-1,0)$ and $v_{n}^{k}=0$ in $\omega \times(M, M+1)$. We also point out that for this construction the assumption $\eta_{\varepsilon} \varepsilon^{1-p} \rightarrow 0$ as $\varepsilon \rightarrow 0$ is needed.

By (6.61), (6.63), and a standard diagonal extraction argument we find sequences $\left(\hat{u}^{k}\right)_{k} \subset\left(u_{n}^{k}\right)_{n, k}$ and $\left(v^{k}\right)_{k} \subset\left(v_{n}^{k}\right)_{n, k}$ such that $\hat{u}^{k} \rightarrow u$ a.e. in $\Omega$, $v^{k} \rightarrow \chi_{\Omega_{h}}$ in $L^{1}(\Omega)$, and

$\limsup _{k \rightarrow \infty} \int_{\Omega}\left(\left(\left(v^{k}\right)^{2}+\eta_{\varepsilon_{k}}\right) f\left(e\left(\hat{u}^{k}\right)\right)+c_{W}\left(\frac{W\left(v^{k}\right)}{\varepsilon_{k}}+\frac{\varepsilon_{k}}{2}\left|\nabla v^{k}\right|^{2}\right)\right) \mathrm{d} x \leqq \bar{G}(u, h)$. 
By using (6.62) and the fact that $u_{n}^{k}=u_{n}$ for all $k, n \in \mathbb{N}$, we can modify $\left(\hat{u}^{k}\right)_{k}$ as described at the end of the proof of Proposition 6.1 (see below (6.55)): we find a sequence $\left(u^{k}\right)_{k}$ which satisfies $u^{k}=u_{0}$ on $\omega \times(-1,0)$, converges to $u$ a.e. in $\Omega$, and (6.64) still holds, that is, $\lim _{\sup _{k \rightarrow \infty}} G_{\varepsilon_{k}}\left(u^{k}, v^{k}\right) \leqq \bar{G}(u, h)$. This concludes the proof.

Acknowledgements. Vito Crismale is supported by the Marie Skłodowska-Curie Standard European Fellowship No. 793018. Manuel Friedrich acknowledges support by the DFG Project FR 4083/1-1 and by the Deutsche Forschungsgemeinschaft (DFG, German Research Foundation) under Germany's Excellence Strategy EXC 2044 -390685587, Mathematics Münster: Dynamics-Geometry-Structure.

Publisher's Note Springer Nature remains neutral with regard to jurisdictional claims in published maps and institutional affiliations.

\section{Compliance with Ethical Standards}

Conflict of interest and ethical statement The authors declare that they have no conflict of interest and guarantee the compliance with the Ethics Guidelines of the journal.

\section{A. Auxiliary Results}

In this appendix, we prove two technical approximation results employed in Sections 5 and 6, based on tools from [17].

Proof. Let $(v, H)$ be given as in the statement of the lemma. Clearly, it suffices to prove the following statement: for every $\eta>0$, there exists $\left(v^{\eta}, H^{\eta}\right) \in$ $L^{p}\left(\Omega ; \mathbb{R}^{d}\right) \times \mathfrak{M}(\Omega)$ with the regularity and the properties required in the statement of the lemma (in particular, $v^{\eta}=u_{0}$ in a neighborhood $V^{\eta} \subset \Omega$ of $\partial_{D} \Omega$ ), such that, for a universal constant $C$, one has $\bar{d}\left(v^{\eta}, v\right) \leqq C \eta$ (cf. (3.13) for $\bar{d}$ ), $\mathcal{L}^{d}\left(H \triangle H^{\eta}\right) \leqq C \eta$, and

$$
\bar{F}_{\text {Dir }}^{\prime}\left(v^{\eta}, H^{\eta}\right) \leqq \bar{F}_{\text {Dir }}^{\prime}(v, H)+C \eta .
$$

We start by recalling the main steps of the construction in [17, Theorem 5.5] and we refer to [17] for details (see also [18, Section 4, first part]). Based on this, we then explain how to construct $\left(v^{\eta}, H^{\eta}\right)$ simultaneously, highlighting particularly the steps needed for constructing $H^{\eta}$.

Let $\varepsilon>0$, to be chosen small with respect to $\eta$. By using the assumptions on $\partial \Omega$ given before (2.4), a preliminary step is to find cubes $\left(Q_{j}\right)_{j=1}^{J}$ with pairwise disjoint closures and hypersurfaces $\left(\Gamma_{j}\right)_{j=1}^{J}$ with the following properties: each $Q_{j}$ is centered at $x_{j} \in \partial_{N} \Omega$ with sidelength $\varrho_{j}, \operatorname{dist}\left(Q_{j}, \partial_{D} \Omega\right)>d_{\varepsilon}>0$ with $\lim _{\varepsilon \rightarrow 0} d_{\varepsilon}=0$, and

$$
\mathcal{H}^{d-1}\left(\partial_{N} \Omega \backslash \widehat{Q}\right)+\mathcal{L}^{d}(\widehat{Q}) \leqq \varepsilon, \quad \text { for } \widehat{Q}:=\bigcup_{j=1}^{J} \bar{Q}_{j} .
$$


Moreover, each $\Gamma_{j}$ is a $C^{1}$-hypersurface with $x_{j} \in \Gamma_{j} \subset \bar{Q}_{j}$,

$$
\mathcal{H}^{d-1}\left(\left(\partial_{N} \Omega \triangle \Gamma_{j}\right) \cap \overline{Q_{j}}\right) \leqq \varepsilon\left(2 \varrho_{j}\right)^{d-1} \leqq \frac{\varepsilon}{1-\varepsilon} \mathcal{H}^{d-1}\left(\partial_{N} \Omega \cap \overline{Q_{j}}\right),
$$

and $\Gamma_{j}$ is a $C^{1}$-graph with respect to $v_{\partial \Omega}\left(x_{j}\right)$ with Lipschitz constant less than $\varepsilon / 2$. (We can say that $\partial_{N} \Omega \cap Q_{j}$ is "almost" the intersection of $Q_{j}$ with the hyperplane passing through $x_{j}$ with normal $v_{\partial \Omega}\left(x_{j}\right)$.) We can also guarantee that

$$
\mathcal{H}^{d-1}\left(\left(\partial^{*} H \cup J_{u}\right) \cap \Omega \cap \widehat{Q}\right) \leqq \varepsilon, \quad \mathcal{H}^{d-1}\left(\left(\partial^{*} H \cup J_{u}\right) \cap \partial Q_{j}\right)=0
$$

for all $j=1, \ldots, J$. To each $Q_{j}$, we associate the following rectangles:

$$
\begin{aligned}
& R_{j}:=\left\{x_{j}+\sum_{i=1}^{d-1} y_{i} b_{j, i}+y_{d} v_{j}: y_{i} \in\left(-\varrho_{j}, \varrho_{j}\right), y_{d} \in\left(-3 \varepsilon \varrho_{j}-t,-\varepsilon \varrho_{j}\right)\right\}, \\
& R_{j}^{\prime}:=\left\{x_{j}+\sum_{i=1}^{d-1} y_{i} b_{j, i}+y_{d} v_{j}: y_{i} \in\left(-\varrho_{j}, \varrho_{j}\right), y_{d} \in\left(-\varepsilon \varrho_{j}, \varepsilon \varrho_{j}+t\right)\right\},
\end{aligned}
$$

and $\widehat{R}_{j}:=R_{j} \cup R_{j}^{\prime}$, where $v_{j}=-v_{\partial \Omega}\left(x_{j}\right)$ denotes the generalized outer normal, $\left(b_{j, i}\right)_{i=1}^{d-1}$ is an orthonormal basis of $\left(v_{j}\right)^{\perp}$, and $t>0$ is small with respect to $\eta$. We remark that $\Gamma_{j} \subset R_{j}^{\prime}$ and that $R_{j}$ is a small strip adjacent to $R_{j}^{\prime}$, which is included in $\Omega \cap Q_{j}$. (We use here the notation $j_{j}$ in place of ${ }_{h, N}$ adopted in [17, Theorem 5.5].)

After this preliminary part, the approximating function $u^{\eta}$ was constructed in [17, Theorem 5.5] starting from a given function $u$ through the following three steps:

(i) definition of an extension $\tilde{u} \in G S B D^{p}\left(\Omega+B_{t}(0)\right)$ which is obtained by a reflection argument la Nitsche [54] inside $\widehat{R}_{j}$, equal to $u$ in $\Omega \backslash \bigcup_{j} \widehat{R}_{j}$, and equal to $u_{0}$ elsewhere. This can be done such that, for $t$ and $\varepsilon$ small, there holds (see below $[17,(5.13)]$ )

$$
\int_{\left(\Omega+B_{t}(0)\right) \backslash \Omega}\left|e\left(u_{0}\right)\right|^{p} \mathrm{~d} x+\int_{\widehat{R}}|e(\widetilde{u})|^{p} \mathrm{~d} x+\int_{R}|e(u)|^{p} \mathrm{~d} x+\mathcal{H}^{d-1}\left(J_{\widetilde{u}} \cap \widehat{R}\right) \leqq \eta,
$$

where $R:=\bigcup_{j=1}^{J} R_{j}$ and $\widehat{R}:=\bigcup_{j=1}^{J} \widehat{R}_{j} \cap\left(\Omega+B_{t}(0)\right)$.

(ii) application of Theorem 3.4 on the function $\widetilde{u}^{\delta}:=\widetilde{u} \circ\left(O_{\delta, x_{0}}\right)^{-1}+u_{0}-u_{0} \circ$ $\left(O_{\delta, x_{0}}\right)^{-1}$ (for some $\delta$ sufficiently small) to get approximating functions $\widetilde{u}_{n}^{\delta}$ with the required regularity which are equal to $u_{0} * \psi_{n}$ in a neighborhood of $\partial_{D} \Omega$ in $\Omega$, where $\psi_{n}$ is a suitable mollifier. Here, assumption (2.4) is crucial.

(iii) correcting the boundary values by defining $u^{\eta}$ as $u^{\eta}:=\widetilde{u}_{n}^{\delta}+u_{0}-u_{0} * \psi_{n}$, for $\delta$ and $1 / n$ small enough.

After having recalled the main steps of the construction in [17, Theorem 5.5], let us now construct $v^{\eta}$ and $H^{\eta}$ at the same time, following the lines of the steps (i), (ii), and (iii) above. The main novelty is the analog of step (i) for the approximating sets, while the approximating functions are constructed in a very similar way. For this reason, we do not recall more details from [17, Theorem 5.5].

Step (i). Step (i) for $v^{\eta}$ is the same done before for $u^{\eta}$, starting from $v$ in place of $u$. Hereby, we get a function $\widetilde{v} \in G S B D^{p}\left(\Omega+B_{t}(0)\right)$. 
For the construction of $H^{\eta}$, we introduce a set $\widetilde{H} \subset \Omega+B_{t}(0)$ as follows: in $R_{j}^{\prime}$, we define a set $H_{j}^{\prime}$ by a simple reflection of the set $H \cap R_{j}$ with respect to the common hyperface between $R_{j}$ and $R_{j}^{\prime}$. Then, we let $\widetilde{H}:=H \cup \bigcup_{j=1}^{J}\left(H_{j}^{\prime} \cap(\Omega+\right.$ $\left.\left.B_{t}(0)\right)\right)$. Since $H$ has finite perimeter, also $\widetilde{H}$ has finite perimeter. By (A.2) we get $\mathcal{H}^{d-1}\left(\partial^{*} \widetilde{H} \cap \widehat{R}\right) \leqq \eta / 3$ for $\varepsilon$ small, where as before $\widehat{R}:=\bigcup_{j=1}^{J} \widehat{R}_{j} \cap\left(\Omega+B_{t}(0)\right)$. We choose $\delta, \varepsilon$, and $t$ so small that

$$
\mathcal{H}^{d-1}\left(O_{\delta, x_{0}}\left(\bigcup_{j=1}^{J} \partial R_{j}^{\prime} \backslash \partial R_{j}\right) \cap \Omega\right) \leqq \frac{\eta}{3} .
$$

We let $H^{\eta}:=O_{\delta, x_{0}}(\tilde{H})$. Then, we get $\mathcal{L}^{d}\left(H^{\eta} \triangle H\right) \leqq \eta$ for $\varepsilon$, $t$, and $\delta$ small enough. By (A.1), (A.4), and $\mathcal{H}^{d-1}\left(\partial^{*} \widetilde{H} \cap \widehat{R}\right) \leqq \eta / 3$ we also have (again take suitable $\varepsilon, \delta$ )

$$
\int_{\partial^{*} H^{\eta}} \varphi\left(v_{H^{\eta}}\right) \mathrm{d} \mathcal{H}^{d-1} \leqq \int_{\partial^{*} H \cap\left(\Omega \cup \partial_{D} \Omega\right)} \varphi\left(v_{H}\right) \mathrm{d} \mathcal{H}^{d-1}+\eta .
$$

Moreover, in view of (2.4) and $\operatorname{dist}\left(Q_{j}, \partial_{D} \Omega\right)>d_{\varepsilon}>0$ for all $j, H^{\eta}$ does not intersect a suitable neighborhood of $\partial_{D} \Omega$. Define $\widetilde{v}^{\delta}:=\widetilde{v} \circ\left(O_{\delta, x_{0}}\right)^{-1}+u_{0}-u_{0} \circ$ $\left(O_{\delta, x_{0}}\right)^{-1}$ and observe that the function $\widetilde{v}^{\delta} \chi_{\left(H^{\eta}\right)^{0}}$ coincides with $u_{0}$ in a suitable neighborhood of $\partial_{D} \Omega$. By (A.5), by the properties recalled for $\tilde{u}$, see (A.3), and the fact that $v=v \chi_{H^{0}}$, it is elementary to check that

$$
\bar{F}_{\text {Dir }}^{\prime}\left(\widetilde{v}^{\delta} \chi_{\left(H^{\eta}\right)^{0}}, H^{\eta}\right) \leqq \bar{F}_{\text {Dir }}^{\prime}\left(v \chi_{H^{0}}, H\right)+C \eta=\bar{F}_{\text {Dir }}^{\prime}(v, H)+C \eta
$$

Notice that here it is important to take the same $\delta$ both for $\widetilde{v}^{\delta}$ and $H^{\eta}$, that is to "dilate" the function and the set at the same time.

Step 2. We apply Theorem 3.4 to $\widetilde{v}^{\delta} \chi_{\left(H^{\eta}\right)^{0}}$, to get approximating functions $\widetilde{v}_{n}^{\delta}$ with the required regularity. For $n$ sufficiently large, we obtain $\bar{d}\left(\widetilde{v}_{n}^{\delta} \chi_{\left(H^{\eta}\right)^{0}}, \widetilde{v}^{\delta} \chi_{\left(H^{\eta}\right)^{0}}\right) \leqq$ $\eta$ and

$$
\left|\bar{F}_{\text {Dir }}^{\prime}\left(\widetilde{v}_{n}^{\delta} \chi_{\left(H^{\eta}\right)^{0}}, H^{\eta}\right)-\bar{F}_{\text {Dir }}^{\prime}\left(\widetilde{v}^{\delta} \chi_{\left(H^{\eta}\right)^{0}}, H^{\eta}\right)\right| \leqq \eta
$$

Step 3. Similar to item (ii) above, we obtain $\widetilde{v}_{n}^{\delta}=u_{0} * \psi_{n}$ in a neighborhood of $\partial_{D} \Omega$. Therefore, it is enough to define $v^{\eta}$ as $v^{\eta}:=\widetilde{v}_{n}^{\delta}+u_{0}-u_{0} * \psi_{n}$. Then by (A.6) and Step 2 we obtain $\bar{d}\left(v^{\eta}, v\right) \leqq C \eta$ and $\bar{F}_{\text {Dir }}^{\prime}\left(v^{\eta}, H^{\eta}\right) \leqq \bar{F}_{\text {Dir }}^{\prime}(v, H)+C \eta$ for $n$ sufficiently large.

We now proceed with the proof of Lemma 6.6 which relies strongly on [17, Theorem 3.1]. Another main ingredient is the following Korn-Poincaré inequality in $G S B D^{p}$, see [15, Proposition 3].

Proposition A.1. Let $Q=(-r, r)^{d}, Q^{\prime}=(-r / 2, r / 2)^{d}$, $u \in G S B D^{p}(Q), p \in$ $[1, \infty)$. Then there exist a Borel set $\omega \subset Q^{\prime}$ and an affine function $a: \mathbb{R}^{d} \rightarrow \mathbb{R}^{d}$ with $e(a)=0$ such that $\mathcal{L}^{d}(\omega) \leqq c r \mathcal{H}^{d-1}\left(J_{u}\right)$ and

$$
\int_{Q^{\prime} \backslash \omega}\left(|u-a|^{p}\right)^{1^{*}} \mathrm{~d} x \leqq c r^{(p-1) 1^{*}}\left(\int_{Q}|e(u)|^{p} \mathrm{~d} x\right)^{1^{*}} .
$$


If additionally $p>1$, then there exists $q>0$ (depending on $p$ and $d$ ) such that, for a given mollifier $\varphi_{r} \in C_{c}^{\infty}\left(B_{r / 4}\right), \varphi_{r}(x)=r^{-d} \varphi_{1}(x / r)$, the function $w=u \chi_{Q^{\prime} \backslash \omega}+a \chi_{\omega}$ obeys

$$
\int_{Q^{\prime \prime}}\left|e\left(w * \varphi_{r}\right)-e(u) * \varphi_{r}\right|^{p} \mathrm{~d} x \leqq c\left(\frac{\mathcal{H}^{d-1}\left(J_{u}\right)}{r^{d-1}}\right)^{q} \int_{Q}|e(u)|^{p} \mathrm{~d} x,
$$

where $Q^{\prime \prime}=(-r / 4, r / 4)^{d}$. The constant in (A.7) depends only on $p$ and $d$, the one in (A.8) also on $\varphi_{1}$.

Proof of Lemma 6.6. We recall the definition of the hypercubes

$$
\begin{aligned}
q_{z}^{k} & :=z+\left(-k^{-1}, k^{-1}\right)^{d}, \quad \tilde{q}_{z}^{k}:=z+\left(-2 k^{-1}, 2 k^{-1}\right)^{d}, \\
Q_{z}^{k} & :=z+\left(-5 k^{-1}, 5 k^{-1}\right)^{d},
\end{aligned}
$$

where in addition to the notation in (6.18), we have also defined the hypercubes $\tilde{q}_{z}^{k}$. In contrast to [17, Theorem 3.1], the cubes $Q_{z}^{k}$ have sidelength $10 k^{-1}$ instead of $8 k^{-1}$. This, however, does not affect the estimates. We point out that at some points in [17, Theorem 3.1] cubes of the form $z+\left(-8 k^{-1}, 8 k^{-1}\right)^{d}$ are used. By a slight alternation of the argument, however, it suffices to take cubes $Q_{z}^{k}$. In particular it is enough to show the inequality [17, (3.19)] for a cube $Q_{j}$ (of sidelength $10 k^{-1}$ ) in place of $\widetilde{Q}_{j}$ (of sidelength $16 k^{-1}$ ), which may be done by employing rigidity properties of affine functions. Let us fix a smooth radial function $\varphi$ with compact support on the unit ball $B_{1}(0) \subset \mathbb{R}^{d}$, and define $\varphi_{k}(x):=k^{d} \varphi(k x)$. We choose $\theta<(16 c)^{-1}$, where $c$ is the constant in Proposition A.1 (cf. also [17, Lemma 2.12]). Recall (6.19) and set

$$
\mathcal{N}_{k}^{\prime}:=\left\{z \in\left(2 k^{-1}\right) \mathbb{Z}^{d}: q_{z}^{k} \cap(U)^{k} \backslash V \neq \emptyset\right\} .
$$

We apply Proposition A. 1 for $r=4 k^{-1}$, for any $z \in \mathcal{N}_{k}^{\prime}$ by taking $v$ as the reference function and $z+\left(-4 k^{-1}, 4 k^{-1}\right)^{d}$ as $Q$ therein. (In the following, we may then use the bigger cube $Q_{z}^{k}$ in the estimates from above.) Then, there exist $\omega_{z} \subset \tilde{q}_{z}^{k}$ and $a_{z}: \mathbb{R}^{d} \rightarrow \mathbb{R}^{d}$ affine with $e\left(a_{z}\right)=0$ such that by (6.30), (A.7), and Hölder's inequality it holds that

$$
\begin{array}{r}
\mathcal{L}^{d}\left(\omega_{z}\right) \leqq 4 c k^{-1} \mathcal{H}^{d-1}\left(J_{v} \cap Q_{z}^{k}\right) \leqq 4 c \theta k^{-d}, \\
\left\|v-a_{z}\right\|_{L^{p}\left(\tilde{q}_{z}^{k} \backslash \omega_{z}\right)} \leqq 4 c k^{-1}\|e(v)\|_{L^{p}\left(Q_{z}^{k}\right)} .
\end{array}
$$

Moreover, by (6.30) and (A.8) it holds that

$$
\begin{aligned}
& \int_{q_{z}^{k}}\left|e\left(\hat{v}_{z} * \varphi_{k}\right)-e(v) * \varphi_{k}\right|^{p} \mathrm{~d} x \leqq c\left(\mathcal{H}^{d-1}\left(J_{v} \cap Q_{z}^{k}\right) k^{d-1}\right)^{q} \int_{Q_{z}^{k}}|e(v)|^{p} \mathrm{~d} x \\
& \quad \leqq c \theta^{q} \int_{Q_{z}^{k}}|e(v)|^{p} \mathrm{~d} x
\end{aligned}
$$

for $\hat{v}_{z}:=v \chi_{\tilde{q}_{z}^{k} \backslash \omega_{z}}+a_{z} \chi_{\omega_{z}}$ and a suitable $q>0$ depending on $p$ and $d$. Let us set

$$
\omega^{k}:=\bigcup_{z \in \mathcal{N}_{k}^{\prime}} \omega_{z}
$$


We order (arbitrarily) the nodes $z \in \mathcal{N}_{k}^{\prime}$, and denote the set by $\left(z_{j}\right)_{j \in J}$. We define

$$
\widetilde{w}_{k}:= \begin{cases}v & \text { in }\left(\bigcup_{z \in \mathcal{N}_{k}^{\prime}} Q_{z}^{k}\right) \backslash \omega^{k} \\ a_{z_{j}} & \text { in } \omega_{z_{j}} \backslash \bigcup_{i<j} \omega_{z_{i}}\end{cases}
$$

and

$$
w_{k}:=\widetilde{w}_{k} * \varphi_{k} \quad \text { in }(U)^{k} \backslash V .
$$

We have that $w_{k}$ is smooth since $(U)^{k} \backslash V+\operatorname{supp} \varphi_{k} \subset \bigcup_{z \in \mathcal{N}_{k}^{\prime}} \tilde{q}_{z}^{k} \subset U$ (recall (6.19)) and $\left.v\right|_{\tilde{q}_{z}^{k} \backslash \omega^{k}} \in L^{p}\left(\tilde{q}_{z}^{k} \backslash \omega^{k} ; \mathbb{R}^{d}\right)$ for any $z \in \mathcal{N}_{k}^{\prime}$, by (A.9b).

We define the sets $G_{1}^{k}:=\left\{z \in \mathcal{N}_{k}^{\prime}: \mathcal{H}^{d-1}\left(J_{v} \cap Q_{z}^{k}\right) \leqq k^{1 / 2-d}\right\}$ and $G_{2}^{k}:=\mathcal{N}_{k}^{\prime} \backslash G_{2}^{k}$. By $\widetilde{G}_{1}^{k}$ and $\widetilde{G}_{2}^{k}$, respectively, we denote their "neighbors", see [17, (3.11)] for the exact definition. We let

$$
\widetilde{\Omega}_{g, 2}^{k}:=\bigcup_{z \in \widetilde{G}_{2}^{k}} Q_{z}^{k}
$$

It holds that (cf. [17, (3.8), (3.9), (3.12)])

$$
\lim _{k \rightarrow \infty}\left(\mathcal{L}^{d}\left(\omega^{k}\right)+\mathcal{L}^{d}\left(\widetilde{\Omega}_{g, 2}^{k}\right)\right)=0 .
$$

At this point, we notice that the set $E_{k}$ in $[17,(3.8)]$ reduces to $\omega^{k}$ since in our situation all nodes are "good" (see (6.30) and [17, (3.2)]) and therefore $\widetilde{\Omega}_{b}^{k}$ therein is empty.

The proof of (3.1a), (3.1d), (3.1b) in [17, Theorem 3.1] may be followed exactly, with the modifications described just above and the suitable slight change of notation. More precisely, by [17, equation below (3.22)] we obtain

$$
\left\|w_{k}-v\right\|_{L^{p}\left(\left((U)^{k} \backslash V\right) \backslash \omega^{k}\right)} \leqq C k^{-1}\|e(v)\|_{L^{p}(U)}
$$

for a constant $C>0$ depending only on $d$ and $p$, and [17, equation before (3.26)] gives

$$
\begin{gathered}
\int_{\omega^{k}} \psi\left(\left|w_{k}-v\right|\right) \mathrm{d} x \leqq C\left(\int_{\omega^{k} \cup \widetilde{\Omega}_{g, 2}^{k}}(1+\psi(|v|)) \mathrm{d} x+k^{-1 / 2}\right. \\
\left.\int_{U}(1+\psi(|v|)) \mathrm{d} x+k^{-p} \int_{U}|e(v)|^{p} \mathrm{~d} x\right),
\end{gathered}
$$

where $\psi(t)=t \wedge 1$. Combining (A.13)-(A.14), using (A.12), and recalling that $\psi$ is sublinear, we obtain (6.31a). Note that the sequence $R_{k} \rightarrow 0$ can be chosen independently of $v \in \mathcal{F}$ since $\psi(|v|)+|e(v)|^{p}$ is equiintegrable for $v \in \mathcal{F}$.

Moreover, recalling (A.10)-(A.11), we sum [17, (3.34)] for $z=z_{j} \in \widetilde{G}_{2}^{k}$ and [17, (3.35)] for $z=z_{j} \in \widetilde{G}_{1}^{k}$ to obtain

$\int_{(U)^{k} \backslash V}\left|e\left(w_{k}\right)\right|^{p} \mathrm{~d} x \leqq \int_{U}|e(v)|^{p} \mathrm{~d} x+C k^{-q^{\prime} / 2} \int_{U}|e(v)|^{p} \mathrm{~d} x+C \int_{\widetilde{\Omega}_{g, 2}^{k}}|e(v)|^{p} \mathrm{~d} x$

for some $q^{\prime}>0$. This along with (A.12) and the equiintegrability of $|e(v)|^{p}$ shows (6.31b). 


\section{References}

1. Ambrosio, L.: Existence theory for a new class of variational problems. Arch. Ration. Mech. Anal. 111, 291-322, 1990

2. Ambrosio, L., Coscia, A., Dal Maso, G.: Fine properties of functions with bounded deformation. Arch. Ration. Mech. Anal. 139, 201-238, 1997

3. Ambrosio, L., Fusco, N., Pallara, D.: Functions of Bounded Variation and Free Discontinuity Problems. Oxford Mathematical Monographs. The Clarendon Press, New York 2000

4. Asaro, R.J., Tiller, W.A.: Interface morphology development during stress corrosion cracking: Part I: via surface diffusion. Metall. Trans. 3, 1789-1796, 1972

5. Bella, P., Goldman, M., Zwicknagl, B.: Study of island formation in epitaxially strained films on unbounded domains. Arch. Ration. Mech. Anal. 218, 163-217, 2015

6. Bellettini, G., Coscia, A., Dal Maso, G.: Compactness and lower semicontinuity properties in $\operatorname{SBD}(\Omega)$. Math. Z. 228, 337-351, 1998

7. BonACINI, M.: Epitaxially strained elastic films: the case of anisotropic surface energies. ESAIM Control Optim. Calc. Var. 19, 167-189, 2013

8. BonACINI, M.: Stability of equilibrium configurations for elastic films in two and three dimensions. Adv. Calc. Var. 8, 117-153, 2015

9. Bonnetier, E., Chambolle, A.: Computing the equilibrium configuration of epitaxially strained crystalline films. SIAM J. Appl. Math. 62, 1093-1121, 2002

10. BRAIDES, A.: Approximation of free-discontinuity problems, vol. 1694. Lecture Notes in Mathematics. Springer, Berlin 1998

11. Braides, A., Chambolle, A., Solci, M.: A relaxation result for energies defined on pairs set-function and applications. ESAIM Control Optim. Calc. Var. 13, 717-734, 2007

12. Cagnetti, F., Colombo, M., De Philippis, G., Maggi, F.: Essential connectedness and the rigidity problem for Gaussian symmetrization. J. Eur. Math. Soc. (JEMS) 19, 395-439, 2017

13. Cagnetti, F., Scardia, L.: An extension theorem in SBV and an application to the homogenization of the Mumford-Shah functional in perforated domains. J. Math. Pures Appl. 9(95), 349-381, 2011

14. Capriani, G.M., Julin, V., Pisante, G.: A quantitative second order minimality criterion for cavities in elastic bodies. SIAM J. Math. Anal. 45, 1952-1991, 2013

15. Chambolle, A., Conti, S., Francfort, G.A.: Korn-Poincaré inequalities for functions with a small jump set. Indiana Univ. Math. J. 65, 1373-1399, 2016

16. Сhambolle, A., Conti, S., Iurlano, F.: Approximation of functions with small jump sets and existence of strong minimizers of Griffith's energy. J. Math. Pures Appl. 9(128), 119-139, 2019

17. Chambolle, A., Crismale, V.: A Density Result in $\mathrm{GSBD}^{p}$ with Applications to the Approximation of Brittle Fracture Energies. Arch. Ration. Mech. Anal. 232, 1329-1378, 2019

18. Chambolle, A., Crismale, V.: Phase-field approximation of a class of cohesive fracture energies with an activation threshold. Adv. Calc. Var. https://doi.org/10.1515/acv-20190018

19. Chambolle, A., Crismale, V.: Compactness and lower semicontinuity in GSBD. J. Eur. Math. Soc. (JEMS). Preprint arXiv:1802.03302. 2018.

20. Снаmbolle, A., Solci, M.: Interaction of a bulk and a surface energy with a geometrical constraint. SIAM J. Math. Anal. 39, 77-102, 2007

21. Conti, S., Focardi, M., Iurlano, F.: Which special functions of bounded deformation have bounded variation? Proc. Roy. Soc. Edinburgh Sect. A 2016

22. Conti, S., Focardi, M., Iurlano, F.: Integral representation for functionals defined on $S B D^{p}$ in dimension two. Arch. Ration. Mech. Anal. 223, 1337-1374, 2017

23. Conti, S., Focardi, M., IURlano, F.: Existence of strong minimizers for the Griffith static fracture model in dimension two. Ann. Inst. H. Poincaré Anal. Non Linéaire, 36, 455-474, 2019 
24. Conti, S., Focardi, M., IURLano, F.: Approximation of fracture energies with $p$-growth via piecewise affine finite elements. ESAIM Control Optim. Calc. Var., 25, Art.34. (2019)

25. Crismale, V.: On the approximation of $S B D$ functions and some applications. SIAM J. Math. Anal. 51, 5011-5048, 2019

26. Dal Maso, G.: An introduction to $\Gamma$-convergence, vol. 8. Progress in Nonlinear Differential Equations and their ApplicationsBirkhäuser Boston Inc, Boston, MA 1993

27. Dal MAso, G.: Generalised functions of bounded deformation. J. Eur. Math. Soc. (JEMS) 15, 1943-1997, 2013

28. Dal Maso, G., Francfort, G.A., Toader, R.: Quasistatic crack growth in nonlinear elasticity. Arch. Ration. Mech. Anal. 176, 165-225, 2005

29. Davoli, E., Piovano, P.: Derivation of a heteroepitaxial thin-film model, Interfaces Free Bound.

30. Davoli, E., Piovano, P.: Analytical validation of the Young-Dupré law for epitaxiallystrained thin films. Math. Models Methods Appl. Sci. 29, 2183-2223, 2019

31. De Giorgi, E., Ambrosio, L.: New functionals in the calculus of variations. Atti Accad. Naz. Lincei Rend. Cl. Sci. Fis. Mat. Natur. (8), 82(1988), 199-210, 1989.

32. Focardi, M., Gelli, M.S.: Asymptotic analysis of Mumford-Shah type energies in periodically perforated domains. Interfaces Free Bound. 9, 107-132, 2007

33. Focardi, M., Gelli, M.S.: Relaxation of free-discontinuity energies with obstacles. ESAIM Control Optim. Calc. Var. 14, 879-896, 2008

34. Fonseca, I., Fusco, N., Leoni, G., Millot, V.: Material voids in elastic solids with anisotropic surface energies. J. Math. Pures Appl. 9(96), 591-639, 2011

35. Fonseca, I., Fusco, N., Leoni, G., Morini, M.: Equilibrium configurations of epitaxially strained crystalline films: existence and regularity results. Arch. Ration. Mech. Anal. 186, 477-537, 2007

36. Fonseca, I., Fusco, N., Leoni, G., Morini, M.: Motion of three-dimensional elastic films by anisotropic surface diffusion with curvature regularization. Anal. PDE 8, 373423,2015

37. Fonseca, I., Pratelli, A., Zwicknagl, B.: Shapes of epitaxially grown quantum dots. Arch. Ration. Mech. Anal. 214, 359-401, 2014

38. FRIEDRICH, M.: A derivation of linearized Griffith energies from nonlinear models. Arch. Ration. Mech. Anal. 225, 425-467, 2017

39. Friedrich, M.: A Korn-type inequality in SBD for functions with small jump sets. Math. Models Methods Appl. Sci. 27, 2461-2484, 2017

40. Friedrich, M.: A Piecewise Korn inequality in SBD and applications to embedding and density results. SIAM J. Math. Anal. 50, 3842-3918, 2018

41. Friedrich, M.: A compactness result in $G S B V^{p}$ and applications to $\Gamma$-convergence for free discontinuity problems. Calc. Var. Partial Differential Equations, 58, Art. 86, 31. 2019

42. Friedrich, M., Solombrino, F.: Quasistatic crack growth in 2d-linearized elasticity. Ann. Inst. H. Poincaré Anal. Non Linéaire, 35, 27-64, 2018

43. Fusco, N., Julin, V., Morini, M.: The surface diffusion flow with elasticity in the plane. Commun. Math. Phys. 362, 571-607, 2018

44. Fusco, N., Julin, V., Morini, M.: The surface diffusion flow with elasticity in three dimensions. Preprint CVGMT 4082. 2018

45. FusCo, N., MoRINi, M.: Equilibrium configurations of epitaxially strained elastic films: second order minimality conditions and qualitative properties of solutions. Arch. Ration. Mech. Anal. 203, 247-327, 2012

46. Gao, H., Nix, W.: Surface roughening of heteroepitaxial thin films. Ann. Rev. Mater. Sci. 29, 173-209, 1999

47. Giacomini, A., Ponsiglione, M.: A $\Gamma$-convergence approach to stability of unilateral minimality properties in fracture mechanics and applications. Arch. Ration. Mech. Anal. 180, 399-447, 2006

48. Goldman, M., Zwicknagl, B.: Scaling law and reduced models for epitaxially strained crystalline films. SIAM J. Math. Anal. 46, 1-24, 2014 
49. GRINFELD, M.A.: Instability of the separation boundary between a non-hydrostatically stressed elastic body and a melt. Sov. Phys. Dokl. 31, 831-834, 1986

50. GRINFELD, M.A.: The stress driven instability in elastic crystals: mathematical models and physical manifestations. J. Nonlinear Sci. 3, 35-83, 1993

51. IURLANO, F.: A density result for GSBD and its application to the approximation of brittle fracture energies. Calc. Var. Partial Differ. Equ. 51, 315-342, 2014

52. Kholmatov, S., Piovano, P.: A unified model for stress-driven rearrangement instabilities. Preprint CVGMT 4228, 2019.

53. Kreutz, L., Piovano, P.: Microscopic validation of a variational model of epitaxially strained crystalline films, Preprint CVGMT 4197, 2019.

54. Nitsche, J.A.: On Korn's second inequality. RAIRO Anal. Numér. 15, 237-248, 1981

55. Piovano, P.: Evolution of elastic thin films with curvature regularization via minimizing movements. Calc. Var. Partial Differ. Equ. 49, 337-367, 2014

56. Siegel, M., Miksis, M.J., Voorhees, P.W.: Evolution of material voids for highly anisotropic surface energy. J. Mech. Phys. Solids 52, 1319-1353, 2004

57. Simon, L.: Lectures on geometric measure theory, vol. 3. Proceedings of the Centre for Mathematical Analysis. Australian National University, Australian National University, Centre for Mathematical Analysis, Canberra 1983

58. Spencer, B.J.: Asymptotic derivation of the glued-wetting-layer model and contactangle condition for Stranski-Krastanow islands. Phys. Rev. B 59, 2011-2017, 1999

59. Tемам, R.: Mathematical problems in plasticity, Gauthier-Villars, Paris, 1985. Translation of Problèmes mathématiques en plasticité. Gauthier-Villars, Paris, 1983.

\author{
Vito CRISMAle \\ CMAP, \\ École Polytechnique, \\ 91128 Palaiseau Cedex \\ France. \\ e-mail: vito.crismale@polytechnique.edu \\ and \\ ManUel FriedRICH \\ Applied Mathematics Münster, \\ University of Münster, \\ Einsteinstrasse 62, \\ 48149 Münster \\ Germany. \\ e-mail: manuel.friedrich@uni-muenster.de
}

(Received October 10, 2019 /Accepted March 31, 2020)

Published online April 25, 2020

(C) Springer-Verlag GmbH Germany, part of Springer Nature (2020) 